\title{
Intrinsic flame instabilities in combustors: analytic description of a 1-D resonator model
}

\author{
Mukherjee Nalini Kanta, Shrira Victor \\ School of Computing and Mathematics, Keele University, UK, ST55BG \\ e-mail: n.k.mukherjee@ keele.ac.uk, Phone number: +44 1782733256
}

The study is concerned with theoretical examination of thermo-acoustic instabilities in combustors and focuses on recently discovered 'flame intrinsic modes'. These modes differ qualitatively from the acoustic modes in a combustor. Although these flame intrinsic modes were intensely studied, primarily numerically and experimentally, the instability properties and dependence on the characteristics of the combustor remain poorly understood. Here we investigate analytically the properties of intrinsic modes within the framework of a linearized model of a quarter wave resonator with temperature and cross-section jump across the flame, and a linear $n-\tau$ model of heat release. The analysis of dispersion relation for the eigen-modes of the resonator shows that there are always infinite numbers of intrinsic modes present. In the limit of small interaction index $n$ the frequencies of these modes depend neither on the properties of the resonator, nor on the position of the flame. For small $n$ these modes are strongly damped. The intrinsic modes can become unstable only if $n$ exceeds a certain threshold. Remarkably, on the neutral curve the intrinsic modes become completely decoupled from the environment. Their exact dispersion relation links the intrinsic mode eigen-frequency $\omega^{i}$ with the mode number $m^{i}$ and the time lag $\tau: \omega^{i}=\left(2 m^{i}+1\right)(\pi / \tau)+m \pi / \tau$, where $m=0,+/-1$. The main results of the study follow from the mode decoupling on the neutral curve and include explicit analytic expressions for the exact neutral curve on the $n-\tau$ plane, and the growth/decay rate dependence on the parameters of the combustor in the vicinity the neutral curve. The instability domain in the parameter space was found to have a very complicated shape, with many small islands of instability, which makes it difficult, if not impossible, to map it thoroughly numerically. The analytical results have been verified by numerical examination.

Keywords: Flame intrinsic modes, $n-\tau$ model, combustors, flame stability, thermo-acoustic instabilities.

\section{Introduction}

Instability in modern day combustors is an issue of fundamental interest and major practical concern [1]. The need of lowering emissions pushes engineers to create 'green' combustion systems by enhancing combustion efficiency and lowering emission products. In this context lean premixed pre-vaporized combustors became the most popular way to address this challenge $[2,3]$. The drawback of these combustors is their susceptibility to combustion instability. These instabilities are thermo-acoustic instabilities that can lead to self-sustained oscillations and cause severe damage to the combustor. It has been common to assume that thermo-acoustic instabilities are triggered by the coupling of flame heat release and one of the acoustic modes. The acoustic modes exist even in the absence of the flame. Thus, the overwhelming majority of studies of 
combustion instabilities in last few decades was focused on the understanding of the acoustic modes, e.g. Lieuwen and Yang [4], Poinsot and Veynante [5], Schuller et al. [6] and Dowling et al. $[7,8]$.

However, recently, a major shortcoming of the established paradigm has been identified by Hoeijmakers et al. [9], Hoeijmakers [10] and Bomberg et al. [11]. Hoeijmakers et al. [9] suggested that the flame subsystem in a combustor can give rise to a completely new family of modes, which are often referred to as the flame intrinsic-thermoacoustic modes. Although it would be more appropriate to call them intrinsic thermoacoustic modes of the flame and its nearby environment (burner). For brevity, throughout the paper we will call them just intrinsic modes. Thus, a set of intrinsic modes (which might be unstable) can exist in a combustor, apart from the conventional acoustic modes. Hoeijmakers et al. [9] considered a $n-\tau$ combustor model with gradually varying reflection coefficient at the ends (i.e. +1 to 0 at the closed end and -1 to 0 at the open end) and numerically evaluated intrinsic mode frequencies. Their analytical analysis was confined to an infinite system. The implicit dispersion relation for the flame in an infinite system was found to be, $\varepsilon+1+\theta F(\omega)=0$, where, $F(\omega)$ is flame transfer function, $\varepsilon$ is ratio of specific acoustic impedances and $\theta$ is ratio of temperatures across the flame. The real parts of the intrinsic mode frequencies for an infinite system were found to be $(\pi \pm 2 k \pi) / \tau$, where, $\tau$ is time lag and $k$ is an integer. The explicit expressions for the threshold of instability and the decay rate were given as, $(\varepsilon+1) / \theta$ and $(1 / \tau) \ln [(\varepsilon+1) / n \theta]$, respectively. The analytical results were complemented by numerical analysis of a single intrinsic mode in a resonator. A possible physical explanation of the origin of flame intrinsic modes was suggested to be the flame reaction on the acoustic velocity fluctuations, created by its own heat release fluctuation. In other words, the flame (and its nearby environment) might create its own local, potentially unstable, feedback loop. Independently, Bomberg et al. [11] performed theoretical stability analysis using scattering matrix of the flame and matched their results with experiments (Gentemann and Polifke [12], Kaess et al. [13]) for two combustor setups, one with a laminar flame holder stabilized and another with swirl stabilized turbulent flame. Thermo-acoustic instabilities observed in these combustor setups were interpreted as manifestations of intrinsic flame instability. Thus, it was concluded that the common assumption that in thermo-acoustic instabilities flame heat release always locks onto one of the acoustic modes, needs to be critically reconsidered on a case by case basis. In a parallel study by Emmert et al [14], the stability of intrinsic modes was investigated from the viewpoint of a balance of the acoustic energy across the flame. Direct numerical simulations (DNS) by Courtine et al. [15], Courtine et al. [16] and Silva et al. [17], where a flame placed in an acoustically anechoic environment, have also confirmed that the intrinsic thermo-acoustic feedback is a genuine physical phenomenon, and not just a spurious by-product of simplistic models. Recent study by Emmert et al. [18] has shown that a sum of acoustic and intrinsic modes constitutes the complete set of eigen-modes of a combustor. A numerical procedure has been developed to compute the acoustic and intrinsic eigen-modes of a combustor. It was noted that it is sometimes difficult to distinguish the intrinsic and acoustic modes, solely, by comparing their mode shapes. It has also been found that increased acoustic losses at the end of combustor may destabilize the combustion system due to 
intrinsic flame instability. A somewhat similar observation has been made in experimental study by Hoeijmakers et al. [19]. The experiments suggest that as the acoustic reflections at the combustor boundaries decrease, the eigen-frequencies of the system becomes fully determined by intrinsic flame modes. The possible explanation was given as: the decrease in upstream and downstream acoustic reflections can put the flame in an open-loop, resulting in exposure of intrinsic instability properties of the flame.

Once the reality of the intrinsic modes has been established beyond any doubt, the following natural questions come to the fore. It would be highly desirable to have an analytical criterion on the appearance of unstable intrinsic modes in a combustor and also, it is important to know the boundaries and topology of the instability domain in the multi-dimensional parameter space of a combustor. Besides, the effects of the combustor properties (e.g., flame location, boundary conditions at the ends, and parameters of the cross-section and temperature jumps) on the intrinsic mode growth rates and positions have to be studied. To grasp the stability behavior of intrinsic modes in a multi-dimensional parameter space via numerics might be extremely time consuming, if not impossible. We reiterate that at present all analytical results for intrinsic modes are confined to anechoic environment, only. Overall, an integrated picture of the instabilities of acoustic and intrinsic modes is needed. The present work addresses this need by focusing on the intrinsic mode part of the picture.

Here we consider a standard 1-D acoustic model of a closed-open combustor with a heat source. The heat release rate is modeled by the linear $n-\tau$ law [20]. Within the framework of this simplified model we provide an overall picture of the intrinsic modes and explicit analytical expressions for the parameters of the intrinsic modes (growth rates, neutral curves, frequencies of unstable modes) for the whole range of the system parameters. We also show that whatever are the properties of the combustor, including the end conditions, there is always infinite number of intrinsic modes present. For small $n$, we derive explicit universal dispersion relation for intrinsic modes. These modes strongly decay for small $n$, which makes them practically impossible to observe. In closed-open combustors these modes can become unstable with increase of $n$. The main discovery is that on the neutral curve the transcendental dispersion relation can be factorized. We call this phenomenon decoupling. Factorization means that the intrinsic modes become completely decoupled from the environment, i.e. it does not feel the properties of the combustor. This allows us to treat this problem analytically and to derive all the characteristics of intrinsic mode instabilities. The instability domain in multi-dimensional parameter space proved to be so complicated that it would have been close to impossible to retrieve it numerically.

The paper is organized as follows. In \$2 we introduce our model of a quarter wave resonator with $n-\tau$ model of heat release and derive a dispersion relation for small amplitude oscillations. The dispersion relation takes into account the effects of cross-section and temperature jumps across the flame. It forms the basis of the subsequent analysis. In \$3, for the sake of completeness, we reproduce the results from Mukherjee et al. [21] and derive an analytical expression for the intrinsic modes in the limit of small interaction index, $n$. In this limit the intrinsic modes are heavily damped. Their frequencies are described by the same analytical expression as for the case of an infinite tube found by Hoeijmakers et al. [9]. The distinctive 
features of acoustic and intrinsic modes are also highlighted and discussed using numerical results. In \$4 we describe decoupling of the intrinsic modes from their environment on the neutral curve and exploit it to derive explicit analytical expressions for the exact intrinsic mode neutral curve, separating stability/instability domains on the $n-\tau$ plane and the growth rate near the neutral curve. We also find exactly the intrinsic mode frequencies on the neutral curve. The analytical results are then compared with numerical simulations within the framework of the dispersion relation obtained in $\$ 2$ and a very good agreement is demonstrated. In \$5 we examine the dependence on parameters (location of the flame, the cross-section and temperature jump) of the intrinsic modes. Finally, in \$6, we summarize our progress in understanding of intrinsic modes in a combustor and outline the remaining open questions.

\section{1-D mathematical model of a resonator}

Throughout this paper we will focus on analytical study of flame intrinsic modes in a common 1-D model of acoustic quarter wave resonator. The previous analytical studies by Hoeijmakers et al. [9] were performed for a tube with anechoic boundary condition. The results of the only earlier analytical study of intrinsic flame modes by Mukherjee et al. [21] will be reproduced in the next section. The acoustic modes in a resonator were extensively studied (see e.g., Lieuwen T., and Yang V. [4]). Here we adopt the basic acoustic model from Schuller et al. [6]. The analytical approach we put forward here can be extended to more complicated combustor setups, as well. However, for simplicity, we will restrict the current analysis to the simplest case of a quarter wave resonator. The 1-D analytical formulation based upon $n-\tau$ model provides an efficient versatile tool enabling us to analyze the combustor modes and gives us a valuable insight into the stability behavior of these modes in multi-dimensional parameter space.

Figure 1 provides a schematic sketch of a quarter wave resonator with a compact heat source at $x=x_{q}$, with $x$ being the longitudinal coordinate with the origin at the closed end of the resonator. $A_{1}, A_{2} / B_{1}, B_{2}$ are the pressure amplitudes for the forward/backward going waves in the upstream and the downstream region, respectively. The mean temperature is assumed to

jump from $T_{1}$ to $T_{2}$ across the flame. The cross sectional area jumps across the flame from $S_{1}$ to $S_{2}$. The effect of mean flow is neglected in this analysis. 


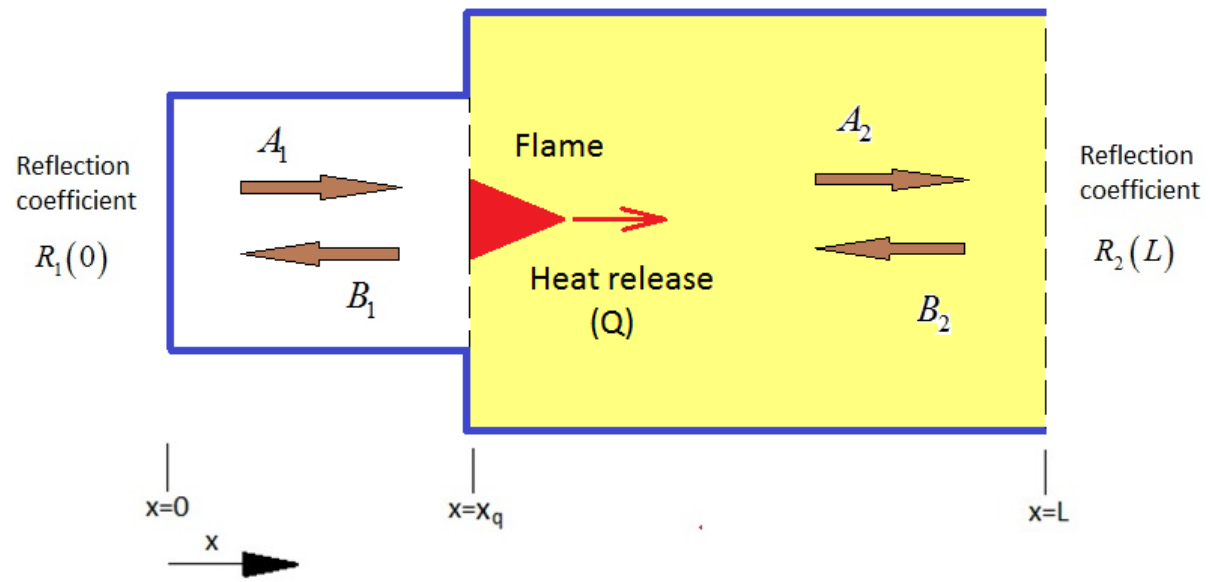

Figure 1: Schematic sketch of a combustor with closed-open end conditions. The combustor has a cross sectional area jump at the flame location $x=x_{q}$. Thick arrows (brown online) symbolize the forward/ backward travelling waves upstream/downstream of the flame. Shaded region (yellow online) marks the domain of higher temperature after the temperature jump across the flame.

The acoustic pressure and particle velocity at the upstream region and at the downstream region of the combustor can be written as (e.g., [22]):

$$
\begin{aligned}
& \tilde{p}_{1}(x, t)=\left[A_{1} e^{i k_{1} x}+B_{1} e^{-i k_{1} x}\right] e^{-i \omega t}, \tilde{u}_{1}(x, t)=\left(\frac{1}{\rho_{1} c_{1}}\right)\left[A_{1} e^{i k_{1} x}-B_{1} e^{-i k_{1} x}\right] e^{-i \omega t}, \text { and } \\
& \tilde{p}_{2}(x, t)=\left[A_{2} e^{i k_{2} x}+B_{2} e^{-i k_{2} x}\right] e^{-i \omega t}, \tilde{u}_{2}(x, t)=\left(\frac{1}{\rho_{2} c_{2}}\right)\left[A_{2} e^{i k_{2} x}-B_{2} e^{-i k_{2} x}\right] e^{-i \omega t} .
\end{aligned}
$$

The wave numbers $k_{i}(i=1,2)$ upstream and downstream of the flame can be presented as $k_{1}=\omega / c_{1}$ and $k_{2}=\omega / c_{2}$, where $\omega$ is the complex frequency, $c_{1}, c_{2}$ represents the speed of sound and $\rho_{1}, \rho_{2}$ are the mean densities upstream and downstream of the flame, respectively. We assume ideal boundary conditions at two ends,

$$
\begin{aligned}
& \text { at } x=0, A_{1} / B_{1}=R_{1}(0)=1, \text { and, } \\
& \text { at } x=L \quad B_{2} e^{-i k L} / A_{2} e^{i k L}=R_{2}(L)=-1 .
\end{aligned}
$$

The pressure and flow rate balance at $x=x_{q}$ implies (Dowling et al. [7], Bauerheim et al. [23]),

$$
\tilde{p}_{1}(x)=\tilde{p}_{2}(x), \text { and } S_{1} \tilde{u}_{1}(x)+\left((\gamma-1) / \rho_{1} c_{1}^{2}\right) \tilde{Q}=S_{2} \tilde{u}_{2}(x)
$$

Here, $\tilde{Q}(t)$ is the heat release rate at $x=x_{q}$ and $\gamma$ represents the ratio of specific heats of air ( $\left.c_{p} / c_{v}\right)$. It is assumed that the heat source acts like a monopole with a volume outflow $(\gamma-1)\left(\tilde{Q} / \rho_{1} c_{1}^{2}\right)$. The linear heat release law can be assumed to be of the form, (see e.g., Truffin and Poinsot [24]) 


$$
\tilde{Q}(t)=\left(\rho_{1} S_{1} c_{1}^{2} /(\gamma-1)\right) n \tilde{u}_{1}(t-\tau) .
$$

Here, $n$ and $\tau$ are the interaction index and time lag, respectively. The rate of heat release fluctuations, $\tilde{Q}$, is assumed to be proportional to the local velocity upstream of the flame, $\tilde{u}_{1}$, with a time lag, $\tau$. In the frequency domain this can be written as,

$$
\tilde{Q}(\omega)=\left(\rho_{1} S_{1} c_{1}^{2} /(\gamma-1)\right) n e^{i \omega \tau} \tilde{u}_{1}(\omega) .
$$

The set of homogeneous equations for $A_{1}, B_{1}, A_{2}$ and $B_{2}$, (3)-(6) is usually presented in the matrix form (e.g., Schuller et al. [6])

$$
\left[\begin{array}{cccc}
1 & -1 & 0 & 0 \\
e^{i k_{1} x_{q}} & e^{-i k_{1} x_{q}} & -e^{i k_{2} x_{q}} & -e^{-i k_{2} x_{q}} \\
\left(1+n e^{i \omega \tau}\right) e^{i k_{1} x_{q}} & \left(-1-n e^{i \omega \tau}\right) e^{-i k_{1} x_{q}} & -\left(\frac{S_{2}}{S_{1}}\right)\left(\frac{\rho_{1} c_{1}}{\rho_{2} c_{2}}\right) e^{i k_{2} x_{q}} & \left(\frac{S_{2}}{S_{1}}\right)\left(\frac{\rho_{1} c_{1}}{\rho_{2} c_{2}}\right) e^{-i k_{2} x_{q}} \\
0 & 0 & -e^{i k_{2} L} & -e^{-i k_{2} L}
\end{array}\right]\left\{\begin{array}{l}
A_{1} \\
B_{1} \\
A_{2} \\
B_{2}
\end{array}\right\}=\left\{\begin{array}{l}
0 \\
0 \\
0 \\
0
\end{array}\right\}
$$

For a nontrivial solution of the eigen-value problem (9) to exist the determinant $D$ of the $4 \times 4$ matrix in (9) has to be zero, which yields $D=0$. Here we, however, find it more convenient to use a compact dispersion relation

$$
\begin{gathered}
{\left[\left(S_{2} / S_{1}\right)\left(\rho_{1} / \rho_{2}\right)\left(c_{1} / c_{2}\right)+1\right] \cos \left(\left(k_{2}-k_{1}\right) x_{q}-k_{2} L\right)+} \\
{\left[\left(S_{2} / S_{1}\right)\left(\rho_{1} / \rho_{2}\right)\left(c_{1} / c_{2}\right)-1\right] \cos \left(k_{2} L-\left(k_{1}+k_{2}\right) x_{q}\right)+2 n e^{i \omega \tau} \sin k_{1} x_{q} \sin k_{2}\left(x_{q}-L\right)=0 .}
\end{gathered}
$$

This compact form of dispersion relation provides the basis of all analytical derivations in the subsequent sections. We denote the function on the left hand side of transcendental equation (10) as $f(\omega)$. Thus, dispersion relation (10) can be re-written compactly as,

$$
f(\omega)=0 .
$$

The dispersion relation can be simplified for special cases worth considering in detail. When there is no cross-section and temperature jumps across the flame, that is $S_{1}=S_{2}$ and $T_{1}=T_{2}$, and thus $c_{1}=c_{2}, \rho_{1}=\rho_{2}$ and $k_{1}=k_{2}=k$, dispersion relation (10) reduces to

$$
\cos k L+n e^{i \omega \tau} \sin k x_{q} \sin k\left(x_{q}-L\right)=0 .
$$

The dispersion relation (12) can be further simplified, when the flame is located exactly in the center of the resonator, i.e. at $x_{q}=L / 2$,

$$
\cos k L=n e^{i \omega \tau} /\left(2+n e^{i \omega \tau}\right) .
$$

The dispersion relation (10), (12) and (13) prescribes the eigen-frequencies $\omega$ of the system in implicit form. Equation (10) describes the most general case (a quarter wave resonator with a cross-section and temperature jump across the flame), while equations (12) and (13) are the 
reduced versions of (10) for special cases. The real part of $\omega$ is the frequency and the imaginary part is the growth/decay rate. Since the dispersion relation in the form (10) is more amenable for analytical study than the commonly used matrix formulation (i.e. the determinant of the $4 \times 4$ matrix in equation (9), equated to zero), we employ it as the starting point for our subsequent analysis. For an insight into the physics of flame intrinsic modes, the particular simplifications of the dispersion relation (12), (13) will be also used. There is no general method enabling one to solve transcendental equations of this type. However, as we show in $\S 4$, the dispersion relation can be factorized on the neutral curves, which allows us to find the neutral curve exactly and approximate solutions in its vicinity.

\section{The place of intrinsic modes in the general picture of the resonator modes}

In this section for completeness we reproduce the results of the analytical study of flame intrinsic modes in a 1-D resonator from Mukherjee et al. [21]. We derive analytical expressions for intrinsic mode frequency and decay rate in the limit of small $n$ and numerically validate the formula.

\subsection{Analytical expression for intrinsic modes in the limit of small $n$ for arbitrary end conditions}

To fix the idea we start with the simplest form of dispersion relation, namely, (13), which provides an implicit expression for all the modes present in the resonator. The presence of flame intrinsic modes, different from the conventional acoustic modes, has been established recently [9]. Both stable and unstable intrinsic modes were observed in experiments and simulations, e.g. $[11,16,17]$. The unstable modes seemingly appear out of nowhere. To understand better the place of intrinsic modes in the big picture it is desirable to know whether these modes exist when they are not unstable, and if yes, where in the complex plane are their frequencies. In this section we address these questions for the case of a quarter wave resonator in the limit of weak flame. From the simplified dispersion relation (13) it is easy to see that for $n \rightarrow 0$ there are only two sets of roots:

(i) the conventional acoustic modes specified by condition $\cos k L=0$, and,

(ii) a new set of roots with large negative imaginary part (this is, $\left|e^{i \omega \tau}\right|>>1$, or, in other words, the strongly damped modes). This can be explained as follows. For nonzero $\cos k L$, there is only one possibility for equation (13) to have solutions, that is the numerator has to be $O(1)$.

This implies that when $n$ is small, $e^{i \omega \tau}$ has to be large. We now apply the same reasoning to the general case.

The dispersion relation can be derived for generic combustors with arbitrary $R_{1}(0)$ and $R_{2}(L)$ retained in the original form in equations (3) and (4), it reads 


$$
\begin{gathered}
(1+\alpha)\left\{e^{i \omega \beta_{1}}-R_{1}(0) R_{2}(L) e^{-i \omega \beta_{1}}\right\}+(1-\alpha)\left\{R_{2}(L) e^{i \omega \beta_{2}}-R_{1}(0) e^{-i \omega \beta_{2}}\right\}+n e^{i \omega \tau} \\
\left\{e^{i \omega \beta_{1}}+R_{2}(L) e^{i \omega \beta_{2}}-R_{1}(0) e^{-i \omega \beta_{2}}-R_{1}(0) R_{2}(L) e^{-i \omega \beta_{1}}\right\}=0
\end{gathered} .
$$

where $\alpha, \beta_{1}$ and $\beta_{2}$ are given by,

$$
\alpha=\left(\frac{S_{2}}{S_{1}}\right)\left(\frac{\rho_{1} c_{1}}{\rho_{2} c_{2}}\right), \beta_{1}=x_{q}\left(\frac{1}{c_{2}}-\frac{1}{c_{1}}\right)-\frac{L}{c_{2}} \text { and } \beta_{2}=\frac{L}{c_{2}}-x_{q}\left(\frac{1}{c_{1}}+\frac{1}{c_{2}}\right) .
$$

To find the roots of the general dispersion relation (14) in the limit of small $n$ we multiply both sides of the resulting equation by $e^{i \omega \beta_{1}}$. This leads to the following expression,

$$
\begin{gathered}
(1+\alpha)\left\{e^{2 i \omega \beta_{1}}-R_{1}(0) R_{2}(L)\right\}+(1-\alpha)\left\{R_{2}(L) e^{i \omega\left(\beta_{1}+\beta_{2}\right)}-R_{1}(0) e^{i \omega\left(\beta_{1}-\beta_{2}\right)}\right\}+n e^{i \omega \tau} \\
\left\{e^{2 i \omega \beta_{1}}+R_{2}(L) e^{i \omega\left(\beta_{1}+\beta_{2}\right)}-R_{1}(0) e^{i \omega\left(\beta_{1}-\beta_{2}\right)}-R_{1}(0) R_{2}(L)\right\}=0
\end{gathered}
$$

By the same reasoning (as for the simplified dispersion relation considered above), the new set of roots (intrinsic modes) for the general dispersion relation (14) must have large negative imaginary part. Thus, for these new roots all the exponents in (16), (that is $2 i \omega \beta_{1}, i \omega\left(\beta_{1}+\beta_{2}\right)$ and $\left.i \omega\left(\beta_{1}-\beta_{2}\right)\right)$ contain highly negative real part, as well. Hence, for small $n, e^{2 i \omega \beta_{1}} \rightarrow 0$, $e^{i \omega\left(\beta_{1}+\beta_{2}\right)} \rightarrow 0, e^{i \omega\left(\beta_{1}-\beta_{2}\right)} \rightarrow 0$. Then dispersion relation (16) reduces to the following simplified form that exhibits decoupling of intrinsic modes from the combustor end conditions $R_{1}(0)$ and $R_{2}(L)$,

$$
\left[(\alpha+1)+n e^{i \omega \tau}\right] R_{1}(0) R_{2}(L)=0 .
$$

Equation (17) shows that in the limit of small $n$ intrinsic modes are so localized, that they do not feel the acoustic boundaries. Equation (17) leads us to an explicit dispersion relation for intrinsic modes in the limit of small $n$, valid for any 1-D combustor obeying $n-\tau$ model of flame heat release,

$$
(\alpha+1)+n e^{i \omega \tau}=0 .
$$

Equation (18) generates the explicit solution for intrinsic mode frequency in the asymptotic limit of small $n$, as follows,

$$
\omega=\left(2 m^{i}+1\right)(\pi / \tau)-(i / \tau) \ln [(\alpha+1) / n] \approx\left(2 m^{i}+1\right)(\pi / \tau)+(i / \tau) \ln (n) .
$$

where, $m^{i}$ is the mode number of the flame intrinsic modes. If we assume the cross-section to be constant, then expression (19a) is identical to the result found by Hoeijmakers et al. [9] for an infinite tube with a flame inside. Note a difference in notation: in the present paper $n$ is the same as in Courtine et al. [16], which relates to $n$ in Hoeijmakers et al. [9] (here labeled as $n_{H}$ ) as $n=\theta n_{H}$, where $\theta=\left(T_{2} / T_{1}\right)-1$. Thus, in the limit of small $n$ intrinsic modes for 1 -D resonator have the same frequencies as those in infinite tube. We stress that the frequencies given by (19b) 
do not depend on any parameters of the resonator including the end conditions. Note, that the intrinsic modes have their own mode numbers, completely independent of the mode numbers of the acoustic modes.

From equation (19b) it is also easy to see that in the limit of small $n$ there is always infinite number of intrinsic modes present in the system for any $n$ and $\tau$. The real part of the flame intrinsic mode frequency depends only on the time lag alone and is given by the expression, $\left(2 m^{i}+1\right)(\pi / \tau)$. The decay rates, however, do not depend on the mode number $\left(m^{i}\right)$. Decay rates are inversely proportional to $\tau$ and logarithmically depend on $n$ and the temperature and cross-section jumps of the resonator. In the asymptotic limit of small $n$, flame intrinsic modes are always strongly damped and independent of the characteristics of the resonator and flame

position. Since the decay rate is high, the intrinsic modes to leading order in $e^{-i k_{1} L}$ do not feel the combustor boundaries. This explains why the result coincides with those for the infinite tube. The practical implication of strong damping of the intrinsic modes in combustors with weak flames is that this feature makes it practically impossible to observe their manifestations in such regimes. Intrinsic modes could only be visible when flame heat release rate is high enough for these modes to approach the neutral curve. In $\S 4.2$ we derive an explicit criterion for occurrence of intrinsic mode instability in the closed-open combustors.

Thus, for small but nonzero $n$ there is always infinite number of flame intrinsic modes present in a combustor, just like there is infinite number of acoustic modes present in a resonator. However, in contrast to nearly neutral acoustic modes, for small $n$ all these modes are strongly damped. They cease to exist for $n=0$, while acoustic modes do not. We have established that in the limit of small $n$ these modes are equally spaced and their frequencies are determined only by the time lag $\tau$ and, correspondingly, do not depend at all on other characteristics of the system. We will show below that in certain bands of $\tau$ the intrinsic modes can become unstable when the value of $n$ exceeds certain threshold. Their behavior in the vicinity of neutral curve will be studied in $\S$ 4. In itself the dispersion relation behavior in the limit of small $n$ is important only in the context of outlining the general picture of intrinsic modes. But its significance becomes apparent in $\S 4$, since the frequencies of intrinsic modes on the neutral curve prove to coincide exactly with the real parts of the eigen-values found in the small $n$ limit.

\subsection{Understanding the stability behavior of intrinsic modes}

Here we examine the features of the full dispersion relation (10) numerically. For certainty, we consider as an example a combustor with the parameters of the test rig at IIT Madras (Vishnu et al. [25]): the length $L$ is $0.75 \mathrm{~m}$, the cross-section $\mathrm{S}$ is $0.0016 \mathrm{~m}^{2}$ (based on $45 \mathrm{~mm}$ inner diameter of the lab scale combustor setup), the temperature $\mathrm{T}$ is constant throughout the duct and equal to $297 \mathrm{~K}$ when there is no flame. 


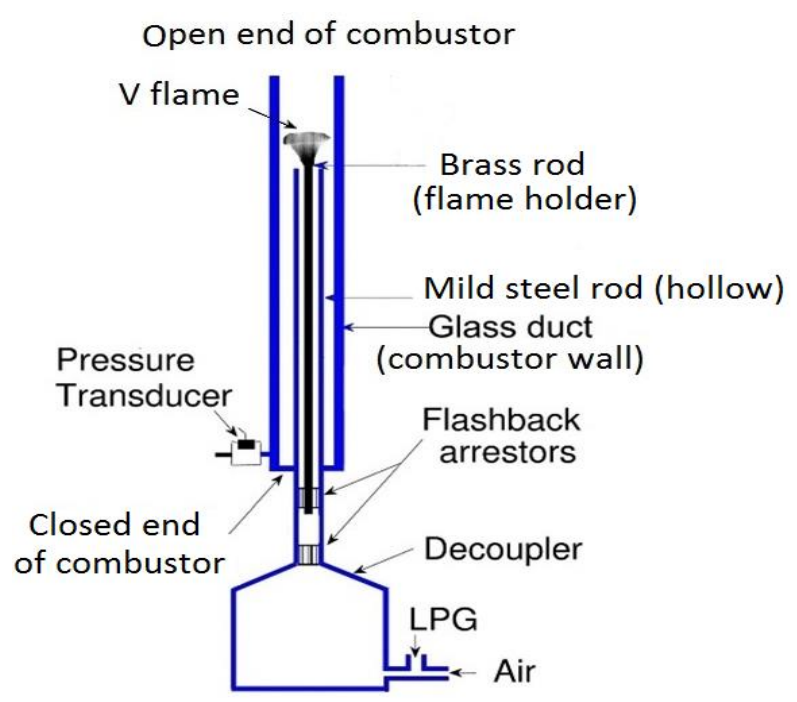

Figure 2: Sketch of the experimental setup (e.g. Vishnu et al. [25] and Mukherjee et al. [26]). The glass duct, marked by thick line (blue online) shows combustor wall. The upper end of the glass duct is open and the lower end is flanged (closed). The flame sits on the tip of a brass rod (shown by thick black line), concentric to the glass duct. There is a hollow mild steel rod (shown by thin line (blue online)) concentric to this glass duct. Supply of air fuel mixture comes via this mild steel rod. The flame can be traversed across the glass duct using a traversing mechanism (not shown in figure). The figure is not to scale.

Figure 2 provides a sketch of the setup. The actual setup consists of a glass duct (shown by thick line (blue online)) with top end open and bottom end closed. Concentric to the duct there is a thin brass rod (shown by black line), at the tip of which flame sits. There is a hollow mild steel rod (shown by thin line (blue online)) concentric to this brass rod, through which air/fuel mixture comes in. In the actual setup, there is a substantial temperature jump across the flame. The flame in the setup can be traversed across the length of the glass duct. For most of our analysis we assume the flame to be located at $x_{q}=L / 2$. However, we stress that the specific parameters of the combustor are immaterial for our study and they are used for illustration only. Our analytical model is valid independently of the combustor specific properties and dimensions, as long as 1-D model idealization and ideal end conditions assumption remain valid.

A valuable insight into the nature of the intrinsic modes can be gained through a set of contour plots of the absolute value of function $f(\omega)$ prescribed in the full dispersion relation $(10,11)$. On the complex $\omega$ plane these plots show topology of $f(\omega)$ and both the frequencies and the growth/decay rates of the modes at the same time. They also facilitate a comparative study of acoustic and intrinsic modes.

In figures 3-6, acoustic modes are marked with diamonds, while intrinsic modes are shown by triangles. The effect of temperature and cross-section jump across flame is neglected in figures 3-5. Figure 6 shows that taking into account the temperature and cross-section jumps does not make any qualitative difference. For simplicity, the flame is assumed to lie at $x_{q}=L / 2$ (figures $3-6)$. In figures 3-5, the acoustic mode frequencies are $\left(2 m_{a}+1\right)(\pi c / 2 L)$ (for $m_{a}=0,1,2 \ldots$ 
and assuming $c_{1}=c_{2}=c$ ) [27] and have zero growth rates in figure 3 . The instability domain is lightly shaded (marked in yellow online). The iso-lines in the figures 3-6 present the mapping of the absolute value of $f(\omega)$ onto the complex $\omega$ plane. The innermost closed loops of the isolines represent the solution region for modal frequency $\omega$. In figures 3-5, the real part of frequencies of the modes are given both in dimensional form ( $\omega \mathrm{in} \mathrm{rad} / \mathrm{s})$ and, in parallel, nondimensionalized on the fundamental modal frequency of acoustic mode $(\pi c / 2 L$ in $\mathrm{rad} / \mathrm{s})$, i.e. $\operatorname{Re}\left(\omega_{n}\right)=\operatorname{Re}(\omega) /(\pi c / 2 L)$. In figure 6 , the non-dimensionalization is performed by cold resonator frequency of acoustic mode $\left(\pi c_{1} / 2 L\right.$ in $\left.\mathrm{rad} / \mathrm{s}\right)$. The growth rate is nondimensionalized by intrinsic instability frequency, i.e. $\operatorname{Im}\left(\omega_{n}\right)=\operatorname{Im}(\omega) /(\pi / \tau)$. The number of frequencies in the system, indeed, exceeds by far the number of acoustic modes, as was also noted by Emmert et al. [18] in their analysis of a premixed combustor.

To show in the same figure the iso-lines of $|f(\omega)|$ for both acoustic and intrinsic modes with huge difference in the decay rates, we split figure 3 into two panels: for small interaction index $n \quad(n=0.025)$ and time lag, $\tau$ equal to $3 \mathrm{~ms}$. In the chosen frequency range there are three intrinsic modes with strong decay rates, as shown in the bottom panel. These modes are equally spaced and the frequencies of these modes are well predicted by (19a). The upper panel shows acoustic modes, which are neutral for the chosen $n$ and $\tau$. When in figure $4, n$ is increased from 0.025 to 1.0 , this decreases significantly the decay rates of the intrinsic modes.

Comparison of figures 4 and 5 shows that increase of $\tau$ from $3 \mathrm{~ms}$ to $5 \mathrm{~ms}$ reduces the intrinsic mode frequency. At some threshold value of $n$ these intrinsic modes become unstable. For example, the fourth intrinsic mode at $4398 \mathrm{rad} / \mathrm{s}$ becomes unstable when $n$ is 1.0. This instability frequency is the same frequency as predicted by equation (19a) in the asymptotic limit of small $n$. The same is true for the second intrinsic mode. This behavior of intrinsic instability frequency (being the same as in the limit of small $n$ ) is robust: it does not depend on the presence of temperature or cross-section jumps in the system, as illustrated by figure 6 , where temperature and cross-section jump across the flame are taken into account. Even in this case the second intrinsic mode attains instability at the same frequency as predicted by equation (19a) in the asymptotic limit of small $n$. However, this is not the only possibility. In $\S 4.1$, we will show that the intrinsic modes can also attain instability at a frequency shifted by $\pm \pi / \tau$ with respect to the frequency $\left(2 m^{i}+1\right)(\pi / \tau)$ predicted by equation (19a). The intrinsic mode frequency might also shift for the modes remaining linearly stable for all $n$, e.g. the first intrinsic mode in figures 4,5 . Numerical simulations (not shown here) indicate that in certain bands of $\tau$ all intrinsic modes become unstable upon exceeding certain $n$-threshold, the threshold will be derived analytically in $\S 4.2$.

In summary, we can say that intrinsic modes are strongly damped in the limit of small $n$ and in this range are easily distinguishable from acoustic modes. On the complex $\omega$ plane the modes could be always distinguished by tracing their position by varying $n$. The frequency of the 
intrinsic modes depends primarily on $\tau$ and does not depend on any parameters of the combustor (within the framework of the adopted model of the closed-open combustor with ideal end conditions). We stress that the frequency depends on $n$ very weakly. In contrast, the intrinsic modes stability is strongly dependent on $n$. These modes can become unstable upon exceeding a certain threshold value of $n$.
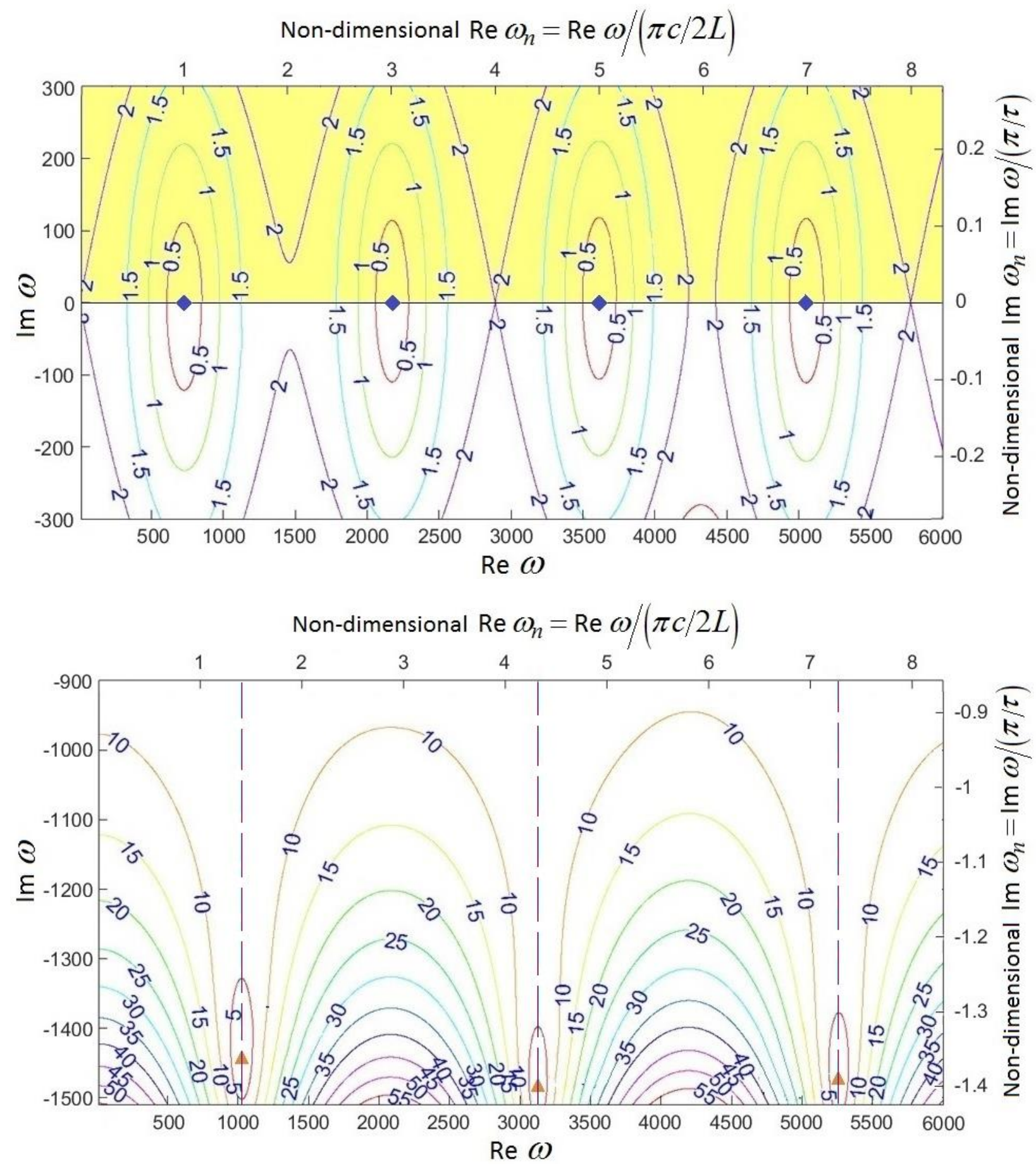
Figure 3: Contour plot of $|f(\omega)|$ for $n=0.025$ and $\tau=3.0 \mathrm{~ms}$ for the particular case $x_{q}=L / 2$ and no temperature and cross-section jump. The parameters used for this plot are: the length $L$ is $0.75 \mathrm{~m}$, the cross-section $\mathrm{S}$ is $0.0016 \mathrm{~m}^{2}$, the temperature $\mathrm{T}$ is constant throughout the duct and equal $297 \mathrm{~K}\left(c_{1}=c_{2}=c=345 \mathrm{~m} / \mathrm{s}\right)$. Two sections are parts of the same contour plot. The domain of instability is lightly shaded (marked in yellow online). Diamonds (blue online) and triangles (orange online) represent the acoustic and intrinsic modes, respectively. Dashed vertical lines indicate the intrinsic mode frequency in the limit of small $n$, given by equation (19a).

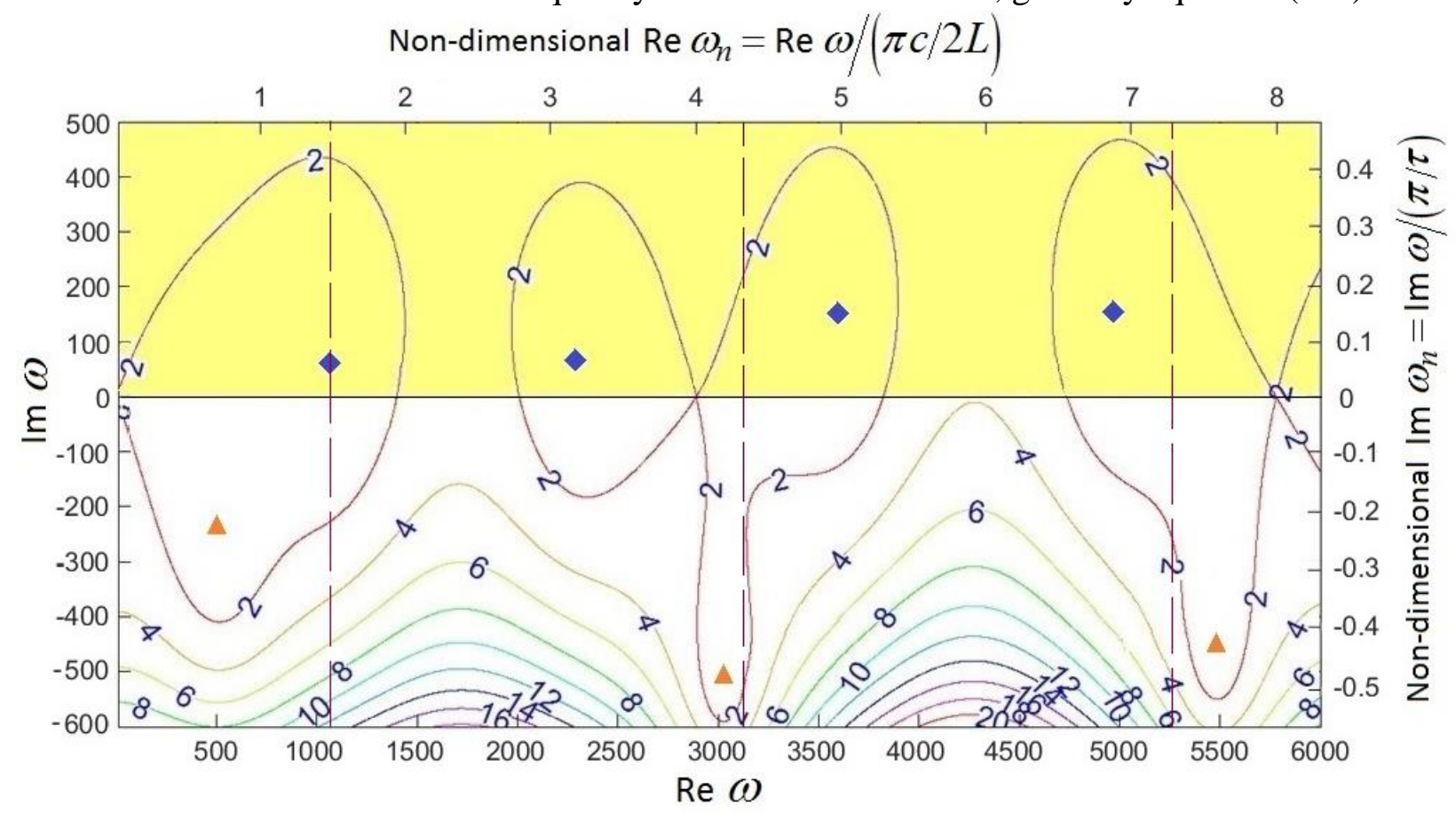

Figure 4: Contour plot of $|f(\omega)|$ for $n=1.0, \tau=3.0 \mathrm{~ms}$. Notation and other parameters are the same as in Fig 3. 


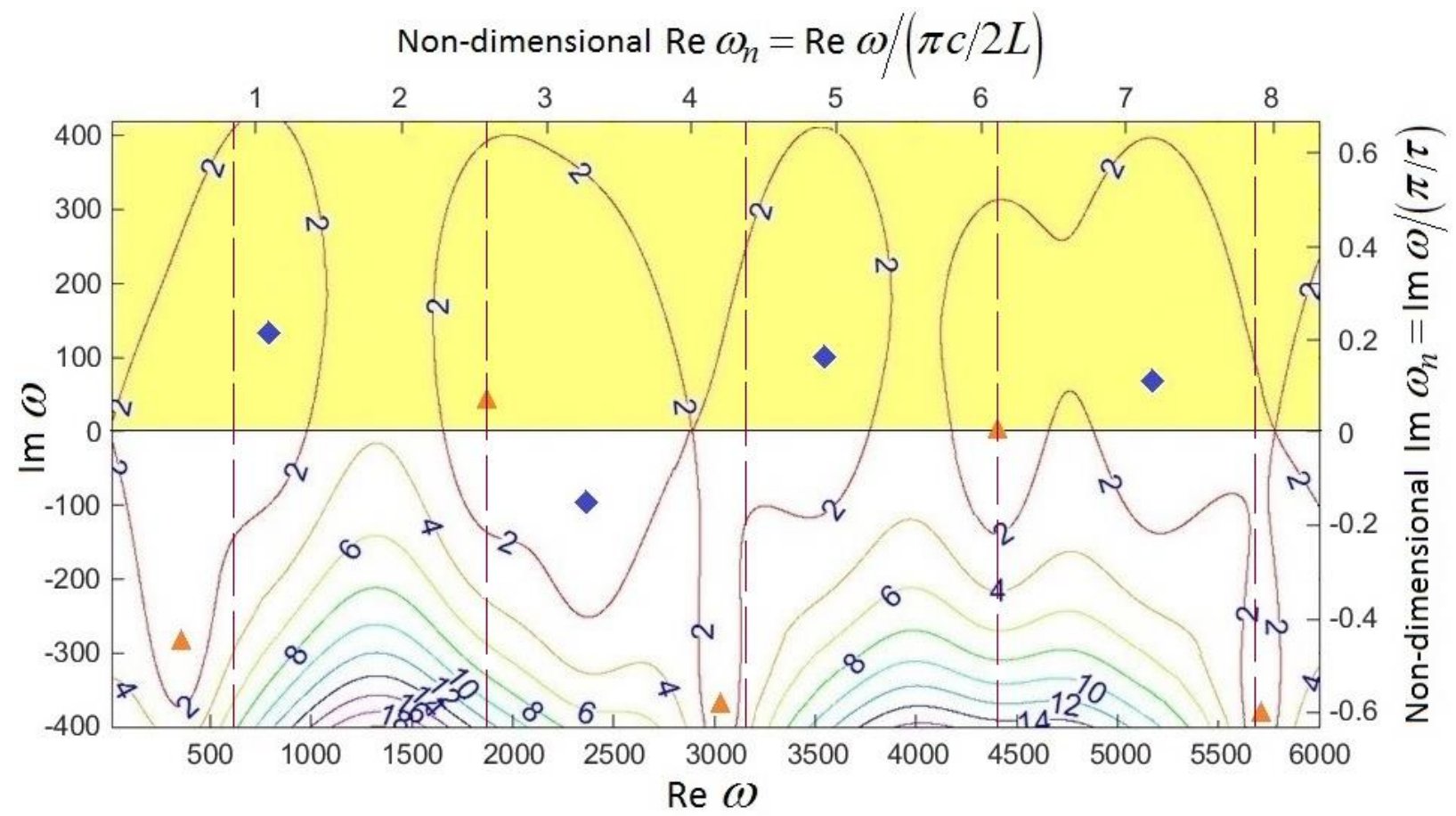

Figure 5: Contour plot of $|f(\omega)|$ for $\tau=5.0 \mathrm{~ms}$ and $n=1.0, x_{q}=L / 2$ and no temperature and cross-section jump. Notation and other parameters are the same as in Fig.3.

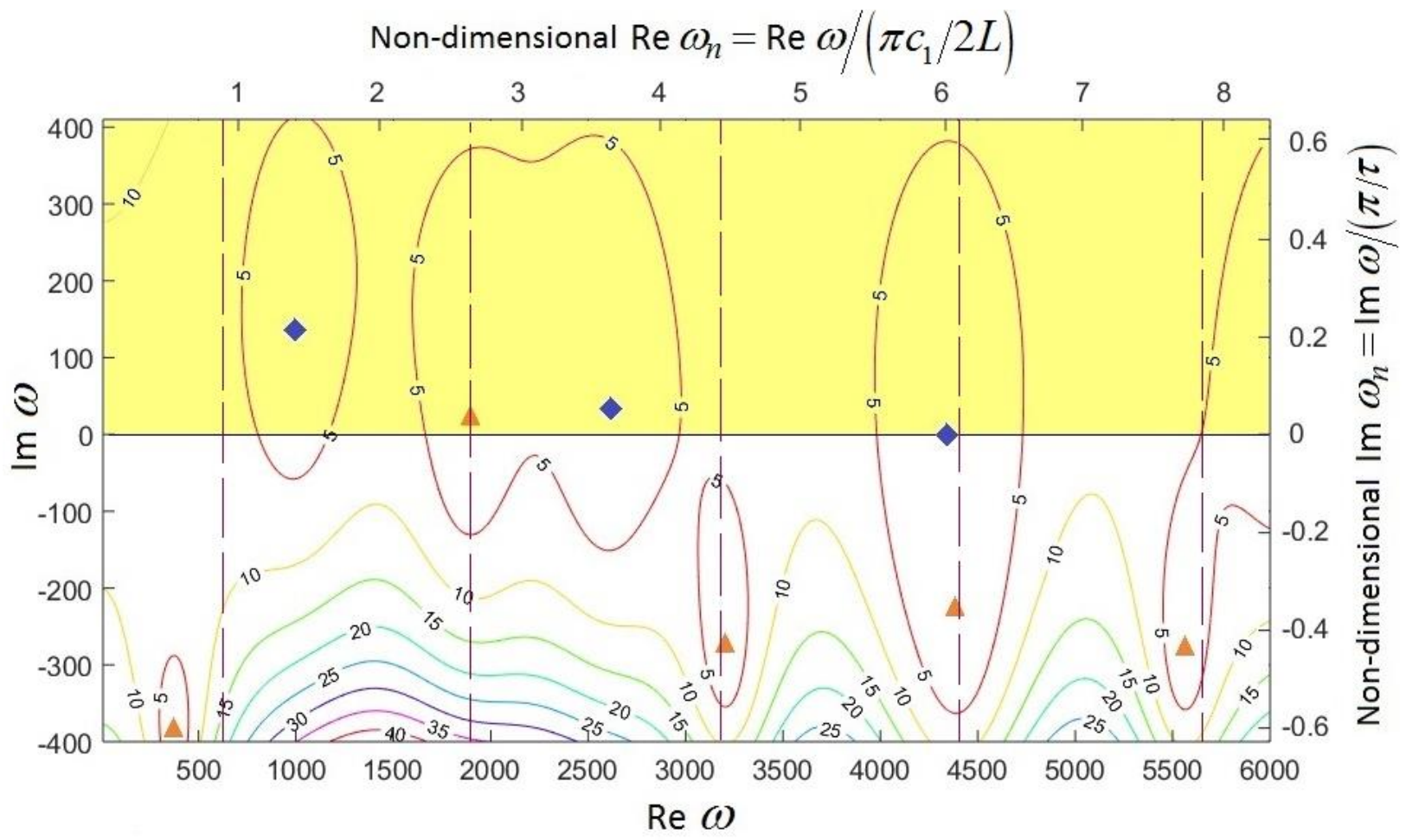

Figure 6: Contour plot of $|f(\omega)|$ for $\tau=5.0 \mathrm{~ms}, n=1.4, x_{q}=L / 2, S_{2} / S_{1}=1.5$ and $T_{2} / T_{1}=2.25$ (or, $\left.c_{2} / c_{1}=1.5\right)$ ). Notation and other parameters are the same as in Fig.3. 


\subsection{Maps of acoustic and intrinsic modes on the $n-\tau$ plane}

The similarities and the differences between acoustic and intrinsic modes can also be elucidated by a set of maps on the $n-\tau$ plane. These maps offer us a lucid picture of the evolution of intrinsic modes in the parameter space and also serve as a validation tool for our analytical results of §3.1. Figures 7 and 8 give examples of such kind of maps for acoustic and intrinsic modes. The acoustic modes are indicated by squares and intrinsic modes by circles. These modal frequencies are tracked down manually on the complex frequency plane (as shown in contour plots 3-6) by changing parameters $n$ and $\tau$. The numerical results are compared with the analytical solution (19a) for small $n$. The effect of temperature and cross-section jump is neglected here (and thus, $c_{1}=c_{2}=c=345 \mathrm{~m} / \mathrm{s}$ ) and the flame is assumed to lie at $x_{q}=L / 2$. In parallel with the dimensional time lag $\tau$ we also use a non-dimensional time lag, $\tau_{n}=\tau c / 2 L$ employing the natural acoustic mode timescale $2 L / c$, where $c$ is the sound speed in the absence of temperature jump. When the fundamental frequencies of acoustic mode $\left(m_{a}=0\right)$ and the intrinsic mode $\left(m^{i}=0\right)$ are the same, i.e. $\pi / \tau=\pi c / 2 L$, this will correspond to $\tau_{n}=1$. Modal frequencies of intrinsic modes are given both in dimensional form ( $f$ in $\mathrm{Hz})$ and, in parallel, non-dimensionalized on the fundamental modal frequency of acoustic mode $(c / 4 L$ in $\mathrm{Hz})$, i.e. $f_{n}=f /(c / 4 L)$.

In figure 7 the time lag $\tau$ varies from $1 \mathrm{~ms}$ to $5 \mathrm{~ms}$. Three different values of $n$ are considered: $n=0.01,0.25$ and 1.0 and the corresponding modal frequencies are obtained using contour plots. The flame intrinsic mode frequencies have $(1 / \tau)$ dependence well outside the limit of small $n$, which is shown by solid line hyperbolae, representing the analytical solution (19a) for small $n$. Multiple hyperbolic lines arise from the same analytical equation (19a). For example, in figure 7 we find that for time lag of 1,2,3,4 and $5 \mathrm{~ms}$, as per equation (19a) the first intrinsic mode frequencies are $500 \mathrm{~Hz}, 250 \mathrm{~Hz}, 166 \mathrm{~Hz}, 125 \mathrm{~Hz}$ and $100 \mathrm{~Hz}$, respectively. These frequencies are connected by the lowest hyperbolic line. Similar exercise is carried out for higher order intrinsic modes, as well. Recall, that the squares and circles in figures 7-8 indicate exact solutions of the full dispersion relation (10) corresponding to the acoustic and intrinsic modes respectively. In the range of small $n$ it is easy to distinguish the acoustic and intrinsic modes. The key implicit assumption employed in drawing these figures is that that the modes identified as acoustic (intrinsic) for small $n$ remain acoustic (intrinsic) when $n$ is increased. This assumption is not always justified. Coupling of acoustic and intrinsic modes might switch identities, i.e. in figures 7-8 the circles (intrinsic modes) and the nearby squares (acoustic modes) might swap positions. This effect is ignored in figures 7-8. Here we did not carry out an extra analysis required to establish whether a particular mode remains acoustic for a chosen value of $n$. Detailed discussion of this phenomenon will be reported in the follow up paper.

In figure $8, \tau$ is fixed at $5 \mathrm{~ms}$ and $n$ is varied from 0 to 1.5 . The corresponding modal frequencies are obtained from the contour plots similar to figures 3-6. When flame is not present in the system, (that is, $n=0$ ), the intrinsic modes are absent, while the frequencies of the 
acoustic modes are $\left(2 m_{a}+1\right)(\pi c / 2 L)$ (for $m_{a}=0,1,2 \ldots$ ) for a quarter wave resonator. As we increase $n$ flame intrinsic modes emerge. The intrinsic mode frequencies are weakly dependent on $n$. In the range of $n$ considered here, the overall change of the intrinsic mode frequency is less than 5\%. To illustrate weak dependence of intrinsic mode frequencies on the resonator length, figure 8 presents results for two different resonator lengths. Note that the significant change in frequency of the fourth intrinsic mode in figure 8 is not due to resonator length, rather it is due to intrinsic-acoustic mode coupling. In this case, due to mode coupling, the acoustic mode becomes unstable at intrinsic mode frequency given by (19a), whereas, the intrinsic mode attains instability at a frequency shifted by $\pi / \tau$ with respect to the frequency (19a). Again, details of this phenomenon will be reported in the follow up paper. However, an insight into the nature of this type of frequency shift caused by mode coupling will be given in $\S 4.1$ and $\S 4.2$.

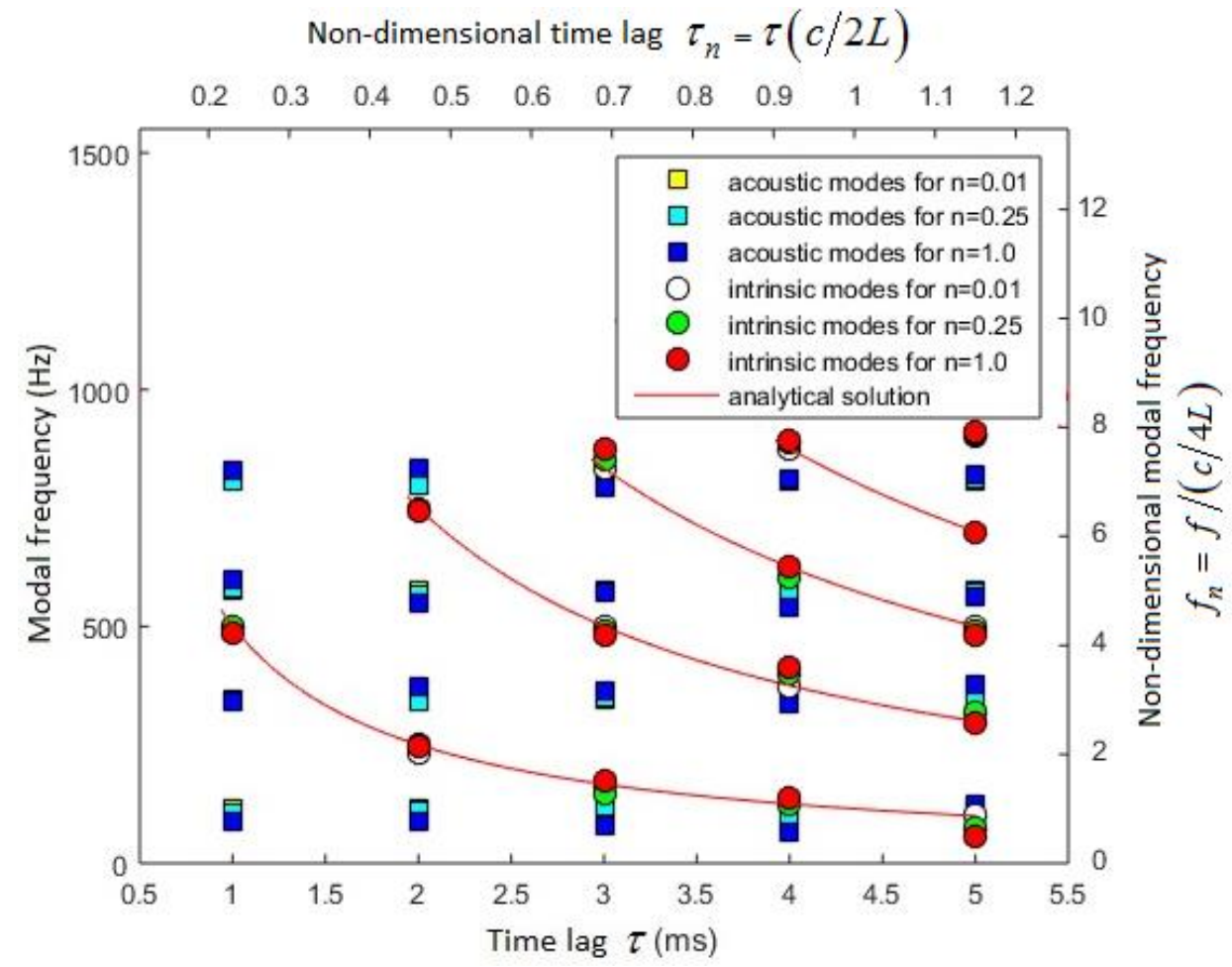

Figure 7: Map of acoustic (squares) and intrinsic (circles) modes: modal frequency vs. time lag $\tau$ for $n=0.01,0.25,1.0, x_{q}=L / 2$ and there is no temperature and cross-section jump. Solid lines show the analytical solution (19a) for small $n$.Other parameters are the same as in Fig.3. 


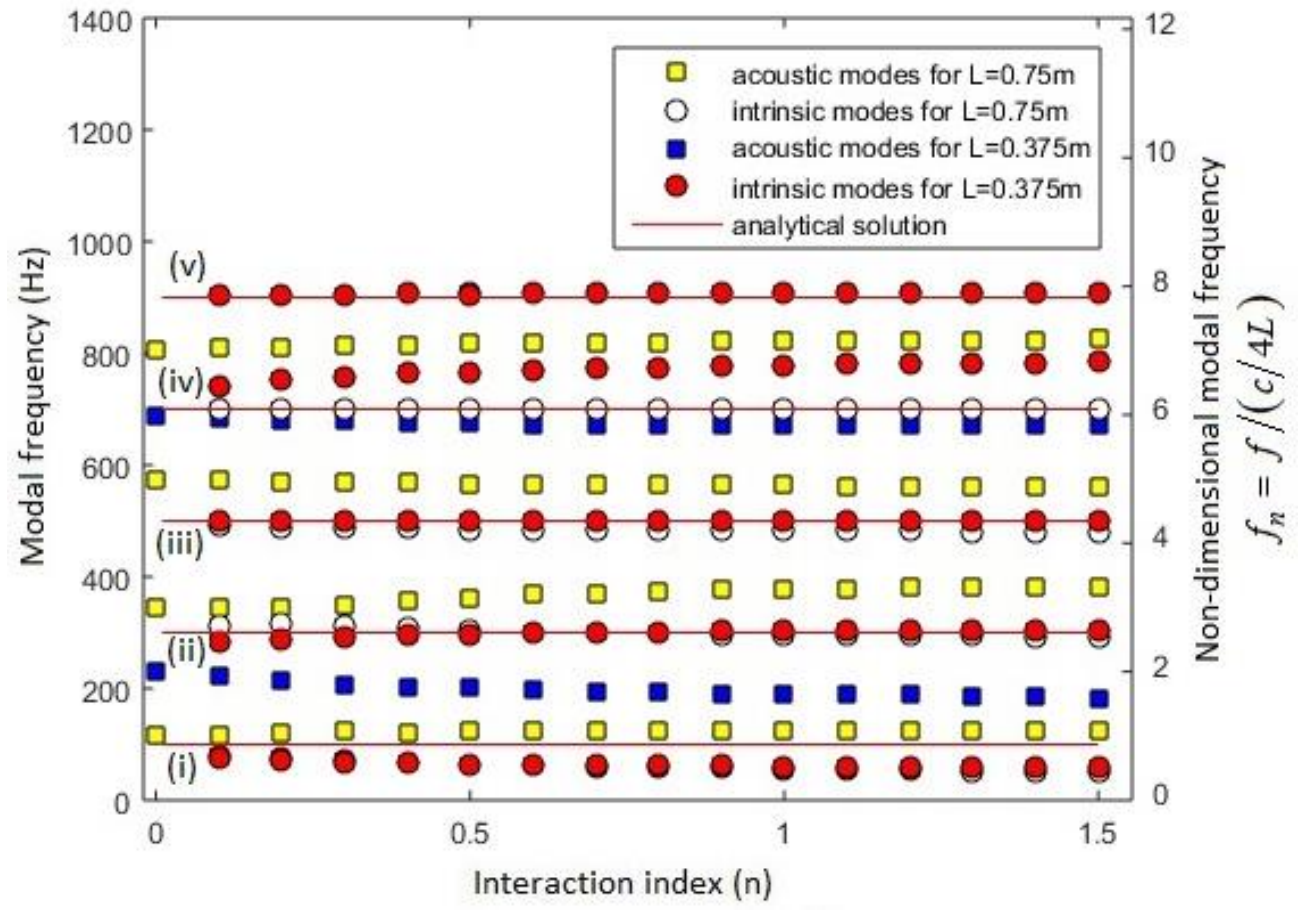

Figure 8: Map of acoustic (squares) and intrinsic modes (circles): modal frequency vs. interaction index $n$. Solid lines show analytical solution (19a) for small $n$. The plot is for: $\tau=5$ ms and two different resonator lengths: $L=0.75 \mathrm{~m}, 0.375 \mathrm{~m}$. The flame is at $x_{q}=L / 2$ and there is no temperature and cross-section jump. The first, second, third, fourth and fifth intrinsic modes are indicated by numbers (i), (ii), (iii), (iv) and (v), respectively.

Crucially, the analytical prediction of the intrinsic mode frequencies (19a) obtained in the asymptotic limit of small $n$ is found to be a very good approximation for a wide range of $n$. A substantial variation of $n$ hardly alters the intrinsic mode frequencies when we have intrinsic mode instability. However, a significant shift of frequency can be visible when we have intrinsicacoustic mode coupling (e.g. the fourth intrinsic mode in figure 8) or when intrinsic mode remains linearly stable for all $n$ (e.g. the first intrinsic mode in figure 8 ). The detailed discussion is beyond the scope of current paper. The intrinsic mode frequency shift by $\pm \pi / \tau$ with respect to the frequency (19a) will be explained in $\S 4.1$.

\section{Intrinsic mode instability: neutral curves and growth rates}

Hoeijmakers et al. [9] found intrinsic modes to be unstable for some values of $n$ and $\tau$. Small $n$ limit of dispersion relation (19b) also suggests that the decay rate of intrinsic modes is logarithmically dependent on $n$. As $n$ increases, the decay rate decreases and at some threshold value of $n$, the decay rate crosses zero, making the intrinsic mode unstable. Here, in $\S 4.1$, we will derive exact analytical expression for the intrinsic mode frequency at the neutral curve. Then, in $\$ 4.2$, will find the exact threshold value of $n$ for intrinsic mode instability, that is, we will find the neutral curve in the $n-\tau$ parameter space. In $\S 4.3$ we derive the growth/decay rates near the neutral curve. The geometry of instability domains for each mode proved to be 
quite complicated. Hence, to simplify handling of infinite number of modes we introduce bounds for the stability domain in $\S 4.4$ and derive simple estimate of the largest growth rate in $\S 4.5$.

\subsection{The frequency of the intrinsic modes at the neutral curve}

In the previous section we have found the intrinsic mode frequency in the limit of small $n$. Here we derive the exact frequency at the neutral curve on the $n-\tau$ plane taking into account crosssection jump, temperature jump and flame location.

\subsubsection{Decoupling on the neutral curve}

The intrinsic modes found analytically for small $n$ can be traced numerically for arbitrary $n$. At first, we denote by $\omega_{c}^{i}$ the discrepancy between the eigen-frequency $\omega^{i}$ and $\omega_{0}^{i}$, the small $n$ prediction given by real part of (19a). We, however, stress that we do not a priori assume $\omega_{c}^{i} / \omega_{0}^{i}$ to be small in our analysis. Thus, for any $n, \operatorname{Re}\left(\omega^{i}\right)=\omega_{0}^{i}+\omega_{c}^{i}$, where superscript $i$ denotes the intrinsic modes. For any value of $n$ the intrinsic mode frequency $\omega$ can be written as, $\omega^{i}=\operatorname{Re}\left(\omega^{i}\right)+i \operatorname{Im}\left(\omega^{i}\right)$, or $\omega^{i}=\left(\omega_{0}^{i}+\omega_{c}^{i}\right)+i \operatorname{Im}\left(\omega^{i}\right)$. On substituting this presentation of $\omega^{i}$ into the full dispersion relation (10), we can re-write it as,

$$
\begin{aligned}
& (\alpha+1) \cos \left\{\left(\omega_{0}^{i}+\omega_{c}^{i}+i \operatorname{Im}\left(\omega^{i}\right)\right) \beta_{1}\right\}+(\alpha-1) \cos \left\{\left(\omega_{0}^{i}+\omega_{c}^{i}+i \operatorname{Im}\left(\omega^{i}\right)\right) \beta_{2}\right\} \\
& +2 n e^{i\left(\omega_{0}^{i}+\omega_{c}^{i}+i \operatorname{Im}\left(\omega^{i}\right)\right) \tau} \sin \left\{\left(\omega_{0}^{i}+\omega_{c}^{i}+i \operatorname{Im}\left(\omega^{i}\right)\right) x_{q} / c_{1}\right\} \sin \left\{\left(\omega_{0}^{i}+\omega_{c}^{i}+i \operatorname{Im}\left(\omega^{i}\right)\right)\left(x_{q}-L\right) / c_{2}\right\}=0
\end{aligned}
$$

where $\alpha, \beta_{1}$ and $\beta_{2}$ are given by equation (15). By virtue of real part of (19a) we have, $e^{i \omega_{0}^{i} \tau}=-1$. Substitution of this identity into (20) simplifies it further. By definition, $\operatorname{Im}\left(\omega^{i}\right)$ is equal to zero for the threshold value of $n$, i.e. $\operatorname{Im}\left(\omega^{i}\right)=0$ at $n=n_{t h}^{i}$. This specifies the neutral curve. Thus, on the neutral curve the imaginary part of (20) reduces to,

$$
2 \operatorname{in}_{t h}^{i} \sin \left(\omega_{c}^{i} \tau\right) \sin \left(k_{1} x_{q}\right) \sin \left(k_{2}\left(x_{q}-L\right)\right)=0 .
$$

Thus, this equation becomes factorized. It is satisfied when just one multiplier containing $\omega_{c}^{i}$ vanishes, that is,

$$
\sin \left(\omega_{c}^{i} \tau\right)=0
$$

This gives us an explicit expression for $\omega_{c}^{i}$,

$$
\omega_{c}^{i}=m \pi / \tau,
$$

where $m$ is an integer (not to be confused with the intrinsic mode number $m^{i}$ ). Once $\omega_{c}^{i}$ is obtained via (23), the frequency for neutral intrinsic mode can be calculated using its definition $\operatorname{Re}\left(\omega^{i}\right)=\omega_{0}^{i}+\omega_{c}^{i}$, which yields (on the neutral curve $\omega^{i}=\operatorname{Re}\left(\omega^{i}\right)$ ), 


$$
\omega^{i}=\left(2 m^{i}+1\right)(\pi / \tau)+(m \pi / \tau) .
$$

On the basis of extensive (although not comprehensive) numerical analysis of the full dispersion relation (10) we hypothesize that the modulus of integer $m$ in (23) does not exceed unity, since $|m|>1$ would imply $\omega_{c}^{i} \tau \geq 2 \pi$. Recall, that according to (19b) the real parts of frequencies of two neighboring intrinsic modes in the limit of small $n$ are separated by $2 \pi / \tau$. Hence, any discrepancy exceeding $2 \pi / \tau$ will imply the change of the mode number. For example, if the third intrinsic mode has the frequency discrepancy exceeding $2 \pi / \tau$, it would have become the second intrinsic mode, while the second intrinsic mode would have become third intrinsic mode. Thus, these modes will exchange their identities. So far, in numerics we have not seen a single instance of such an exchange. Therefore, we assume $\omega_{c}^{i}$ to be confined by the condition prohibiting such an exchange of identities, $-2 \pi / \tau<\omega_{c}^{i}<2 \pi / \tau$. But even if we lift the restriction and allow $\omega_{c}^{i}$ to exceed $2 \pi / \tau$, $\square$ we find that the resulting new neutral curve segments (other than $\omega_{c}^{i} \tau=0, \omega_{c}^{i} \tau=\pi$ and $\omega_{c}^{i} \tau=-\pi$ ) correspond to the neutral curve segments of either higher or lower order intrinsic modes. Thus, from the stability prediction perspective, lifting the restriction $\left|\omega_{c}^{i}\right|<2 \pi / \tau$, yields nothing new compared to what has been already captured in the stability maps obtained under the restriction.

According to (23), on the $m=0$ part of the neutral curve on the $n-\tau$ plane $\omega_{c}^{i}=0$, that is, the frequency exactly equals the value predicted in the limit of small $n$. This remarkable coincidence will be discussed in the next section. Apart from the $m=0$ option, the assumption prohibiting the exchange of identities condensed into condition $-2 \pi / \tau<\omega_{c}^{i}<2 \pi / \tau$, leaves only two other possibilities, namely $m= \pm 1$, which corresponds to intrinsic mode frequency shifts by $\pm \pi / \tau$ with respect to $\omega_{0}^{i}$.

\subsubsection{Why is the instability frequency independent of the resonator parameters on the neutral curve?}

Thus we have found an unexpected remarkable exact result. On the neutral curve the instability frequency is completely independent of all the parameters of the combustor we take into account in our model (the length, the flame location, cross-section jump and temperature jump) except the time lag $\tau$. Here we briefly discuss possible mathematical and physical reasons for such an unusual behavior.

It is easy to see from (21) that at the neutral curve the intrinsic mode deviation $\omega_{c}^{i}$ from $\omega_{0}^{i}$, i.e. its frequency in the asymptotic limit of $n \rightarrow 0$, is completely decoupled from the flame location and temperature jump, because of the equation factorization. Note also that the effect of crosssection jump does not even feature in this equation. This makes the intrinsic mode frequency completely independent of the environment on the neutral curve. In the segment of the neutral 
curve corresponding to $\omega_{c}^{i} \tau=0$, the eigen-frequency is exactly equal to $\omega_{0}^{i}$ and in the segments corresponding to $\omega_{c}^{i} \tau=\pi$ and $\omega_{c}^{i} \tau=-\pi$, there is a frequency deviation of $\pi / \tau$ and $-\pi / \tau$, respectively, compared to $\omega_{0}^{i}$. There is, however, a qualitative difference between the eigenfrequencies in the asymptotic limit of small $n$ and the eigen-frequencies on the neutral curve. In the limit of small $n$, the intrinsic modes are so strongly damped, that they do not feel the combustor end conditions and, hence, the length of the combustor, as well as, the position of the flame. We stress that as per (19b) neither the real part of $\omega^{i}$ nor its imaginary part feels the parameters of the combustor (except $\tau$ ) in the limit of small $n$. It can be seen from the contour plots in figures 3-6 that as $n$ is increasing the real part of the eigen-frequency $\operatorname{Re} \omega^{i}$ for a given combustor is hardly changing. The only change of frequency can be seen when the real parts of frequencies of neighboring intrinsic and acoustic modes are very close (then we have intrinsicacoustic mode coupling scenario) or when the intrinsic mode remains linearly stable for all $n$ (e.g. the first intrinsic mode in figures 4, 5). Hence, the real part remains insensitive to all parameters of the combustor (except $\tau$ ), while the decay rate does depend on all the characteristics of the chosen combustor. When on complex $\omega^{i}$ plane we approach the neutral curve (from either side), the intrinsic modes growth/decay rate by definition tends to zero. Hence, given that on the neutral curve even the weak dependence of $\operatorname{Re} \omega^{i}$ vanishes, the dependence of $\omega^{i}$ on the flame location, cross-section and temperature jump also vanishes. To explain why the dependence of $\operatorname{Re} \omega^{i}$ on all parameters but $\tau$ vanishes on the neutral curve consider our simplified dispersion relation (13) which we repeat for convenience: $\cos k L=n e^{i \omega \tau} /\left(2+n e^{i \omega \tau}\right)$. By definition, on the neutral curve the eigen-frequency $\omega$ is real. Since $k=\omega / c$ and the speed of sound $c$ is also real, this requires $\cos k L$ to be real, as well. This is possible only if $e^{i \omega \tau}$ is real, which implies $\sin (\omega \tau)=0$, resulting in the solution, $\omega_{c}^{i}=m \pi / \tau$, where $m$ is an integer. The same type of reasoning applies to the general form of the dispersion relation (10).

The decoupling phenomenon is not confined to the specific quarter wave resonator we were examining. It can be shown that the decoupling (or, in other words, the factorization of the dispersion relation) holds for other types of combustors of arbitrary length, cross-section and temperature jump with perfectly closed or perfectly open end conditions.

\subsection{Instability threshold for the intrinsic modes}

Here we derive an explicit expression for $n$ as a function of $\tau$ where intrinsic mode becomes unstable. We will refer to this specific function as $n_{t h}^{i}$. On this basis for each intrinsic mode, we will draw a set of neutral curves, i.e. the boundaries of stability domains on the $n-\tau$ plane. These neutral curves will be analyzed below to provide an insight into stability behavior of intrinsic modes. Similar to $\S 4.1$, the analysis in this section takes cross-section jump, temperature jump and flame location into account. 


\subsubsection{The neutral curve: Exact solution}

Making use of $e^{i \omega_{0}^{i} \tau}=-1$, the real part of (20) on the neutral curve instantly yields the threshold $n_{t h}^{i}$

$$
n_{t h}^{i}=\frac{(\alpha+1) \cos \left\{\left(\omega_{0}^{i}+\omega_{c}^{i}\right) \beta_{1}\right\}+(\alpha-1) \cos \left\{\left(\omega_{0}^{i}+\omega_{c}^{i}\right) \beta_{2}\right\}}{2 \cos \left(\omega_{c}^{i} \tau\right) \sin \left(k_{1} x_{q}\right) \sin \left\{k_{2}\left(x_{q}-L\right)\right\}},
$$

where $\alpha, \beta_{1}$ and $\beta_{2}$ are provided by (15). First we examine a special case of $x_{q}=L / 2$. In this case, expression (25) can be significantly simplified. For the segments of the neutral curve corresponding to $\omega_{c}^{i} \tau=0, \omega_{c}^{i} \tau=\pi$ and $\omega_{c}^{i} \tau=-\pi$ we find

$$
n_{t h}^{i}=2 \cos \left(\left(2 m^{i}+1+m\right) \frac{\pi L}{\tau c}\right) /\left[\cos \left(\omega_{c}^{i} \tau\right)\left\{\cos \left(\left(2 m^{i}+1+m\right) \frac{\pi L}{\tau c}\right)-1\right\}\right] .
$$

Where, $m$ is $0,+1$ and -1 for the neutral curve segments $\omega_{c}^{i} \tau=0, \omega_{c}^{i} \tau=\pi$ and $\omega_{c}^{i} \tau=-\pi$, respectively. An equivalent non-dimensional version can be written as,

$$
n_{t h}^{i}=2 \cos \left(\left(2 m^{i}+1+m\right) \frac{\pi}{2 \tau_{n}}\right) /\left[\cos \left(\omega_{c}^{i} \tau\right)\left\{\cos \left(\left(2 m^{i}+1+m\right) \frac{\pi}{2 \tau_{n}}\right)-1\right\}\right] .
$$

Where $\tau_{n}=\tau c / 2 L$. The neutral curve for the second intrinsic mode $\left(m^{i}=1\right)$ is shown in figure 9 as a typical example. Figure 9 is divided into two panels with slightly overlapping ranges in $\tau$. Panel (b) is the blow up of the smaller time lag domain as compared to panel (a). In all subsequent neutral curves, we display both dimensional and non-dimensional scales of time lag. In figure 9 temperature and cross section are uniform and thus, $c_{1}=c_{2}=c=345 \mathrm{~m} / \mathrm{s}$. Figure 9 has two notable features. There is a narrow island of instability shown to the left and a large instability domain confined by neutral curve loop on the right hand side, hereinafter referred as 'neutral loop' or just 'loop' for brevity. The right hand side loop has three distinct segments. They correspond to the branches, $\omega_{c}^{i} \tau=0, \omega_{c}^{i} \tau=\pi$ and $\omega_{c}^{i} \tau=-\pi$ (blue, red and green online). Feature wise the pattern of the instability island on the left is the same as of the larger loop on the right. Moreover, this pattern is repetitive (with diminishing width) towards the left as evident from figure 9 (b). To avoid cluttering the figure, we hid the smallest patterns under hatched lines. As these loops become thinner and thinner, they still manifest themselves as a combination of $\omega_{c}^{i} \tau=0, \omega_{c}^{i} \tau=\pi$ and $\omega_{c}^{i} \tau=-\pi$ segments of the neutral curve. In some loops we have two neutral segments corresponding to $\omega_{c}^{i} \tau=\pi$ and in some loops the sequence of $\omega_{c}^{i} \tau=\pi$ and $\omega_{c}^{i} \tau=-\pi$ is reversed as compared to the main neutral loop on the extreme right. The 'instability domain' is lightly shaded (marked in yellow online). Each neutral loop composed of the neutral curve segments $\omega_{c}^{i} \tau=0, \omega_{c}^{i} \tau=\pi$ and $\omega_{c}^{i} \tau=-\pi$ confines the instability domain from below. For 
small $n$, as we have shown in $\S 3.1$, the intrinsic modes are always strongly decaying; while with increase of $n$ the decay rate decreases until vanishing and then changing sign at the neutral curve. Hence, below the neutral curve there is always the stability domain for intrinsic mode under consideration (it might be unstable for another mode) and above is the instability domain for the chosen mode. This will be independently verified below in \$4.2.2.

For any specific loop, the solid lines represent the neutral curve for intrinsic modes and the dashed lines show their continuations. These continuations are neutral curves segments for acoustic modes coupled to intrinsic modes, since these dashed lines correspond to exact neutral solutions of the full dispersion relation (10) deeply embedded into intrinsic mode stability domain. This interpretation is supported by numerical observations, discussed below in \$4.2.2. The conditions $\omega_{c}^{i} \tau=0$ and $\omega_{c}^{i} \tau=\pi$, as well as, $\omega_{c}^{i} \tau=0$ and $\omega_{c}^{i} \tau=-\pi$ have to be satisfied simultaneously when we have a situation of intrinsic-acoustic mode coupling. For these situations, for the same time lag we have two coupled solutions (one for intrinsic mode and other for coupled acoustic mode) instead of a single solution for intrinsic mode. Situations of intrinsicacoustic mode coupling occur near the intersection point of neutral curve segments $\omega_{c}^{i} \tau=0$ and $\omega_{c}^{i} \tau=\pi$; as well as, $\omega_{c}^{i} \tau=0$ and $\omega_{c}^{i} \tau=-\pi$; in figure 9. When there is no coupling between intrinsic and acoustic modes, there is only one solution corresponding to $\omega_{c}^{i} \tau=0$ for the intrinsic mode. This is the domain lying between the two afore-mentioned intersection points in the neutral loop. Because of intrinsic-acoustic mode coupling we can have an additional domain of instability. However, in this paper this type of instability will not be discussed and in all neutral curves from figure 9 onwards we focus on intrinsic instability domain, only.

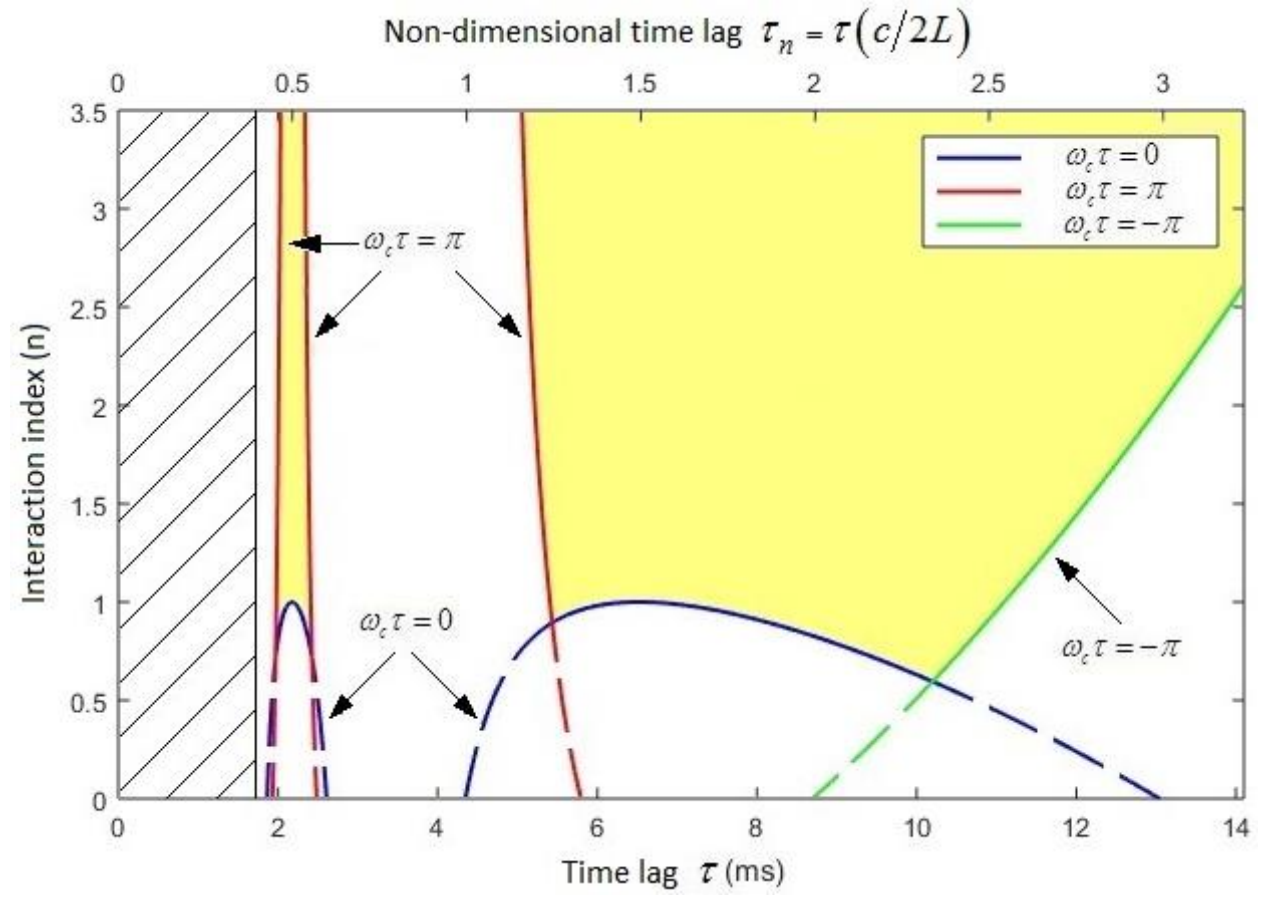

(a) 


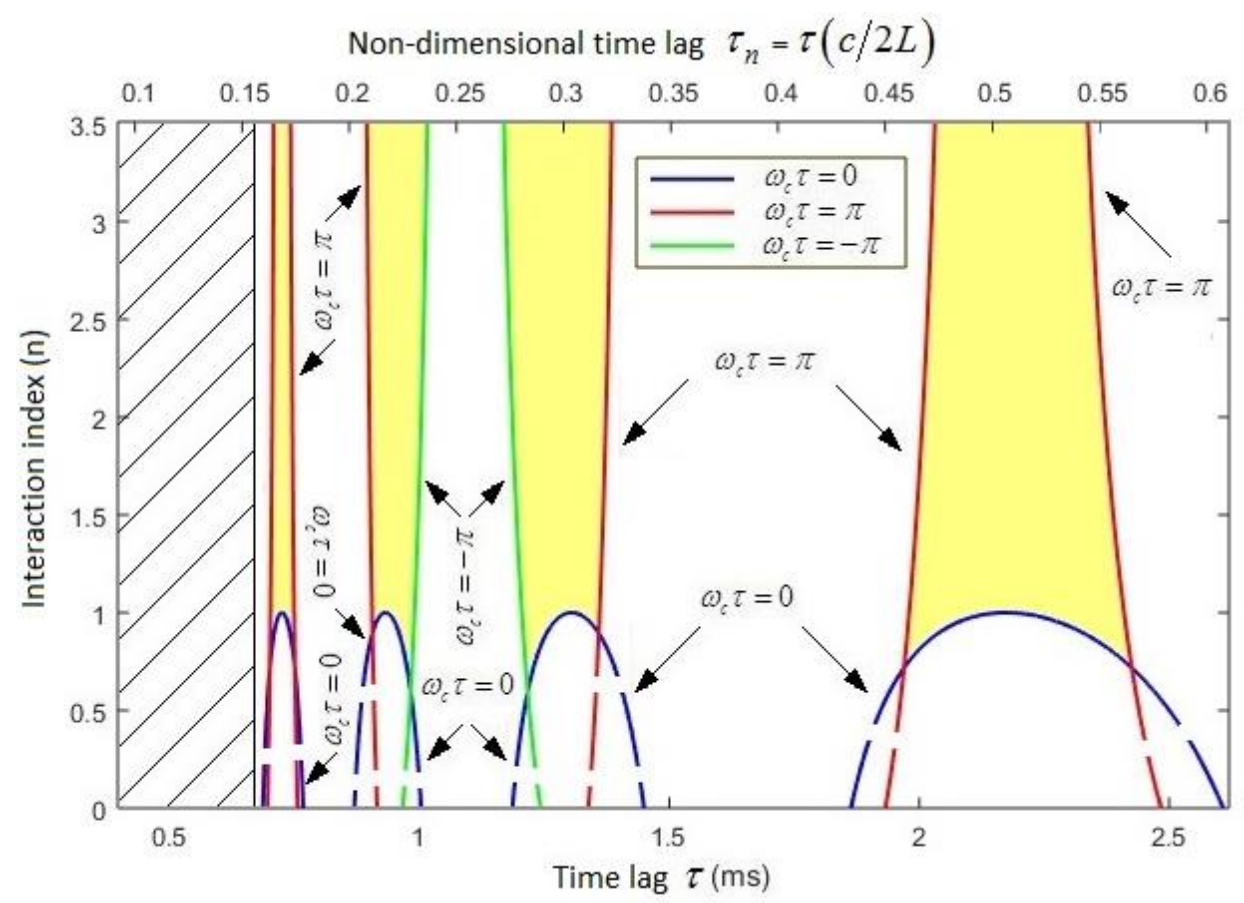

(b)

Figure 9: Neutral curve (26) and stability domain on the $n-\tau$ plane for the second intrinsic mode $\left(m^{i}=1\right)$, when $x_{q}=L / 2$ and there is no temperature and cross-section jump (and thus, $c_{1}$ $=c_{2}=c=345 \mathrm{~m} / \mathrm{s}$ ). Figures (a) and (b) are on a different scale in $\tau$. Figure (b) highlights the neutral curves in the smaller time lag domain as compared to figure (a). The instability domain is lightly shaded (marked in yellow online). The segments $\omega_{c}^{i} \tau=0, \omega_{c}^{i} \tau=\pi$ and $\omega_{c}^{i} \tau=-\pi$ of the neutral curve are indicated by arrows (blue, red and green, respectively, online). The solid lines show segments of the neutral curve for the second intrinsic mode and the dashed lines show their continuations, which are also exact solutions of (26). We interpret them as neutral curves for acoustic modes coupled to the intrinsic mode. Hatched area indicates the domain with multiple instability islands narrowing with the decrease of $\tau$. These islands are difficult to plot and therefore, they are not shown. The other parameters are the same as in figure 3.

The neutral curve segments corresponding to $\omega_{c}^{i} \tau=\pi$ and $\omega_{c}^{i} \tau=-\pi$ yield higher values of $n_{\text {th }}^{i}$ compared to the $\omega_{c}^{i} \tau=0$ segment. The islands of instability in the left hand corner of figure 9 also manifest coupling between acoustic and intrinsic modes. The reason is the same as for the main neutral curve loop on the extreme right in figure 9 (a)). Because these loops are so narrow, that the span of pure intrinsic mode 'uncoupled solution' corresponding to $\omega_{c}^{i} \tau=0$ is also quite narrow. Hence, the points of intersection of neutral curve segments lie close to each other, which implies much stronger role of coupling in this domain of time lag. Detailed analysis of how intrinsic and acoustic mode live together in a combustor and the corresponding overall stability domain due to coupling of modes will be the subject of a follow up paper. However, discussing 
figure 9 we have to mention that there are also isolated nearly vertical segments of the neutral curve we opted not to show in the figure. These segments correspond to $\omega_{c}^{i} \tau=\pi$ and $\omega_{c}^{i} \tau=-\pi$ segments located between the large loop on the right and the first small loop on the left of the figure and are also due to acoustic modes coupled with intrinsic modes.

Note that according to (25), (26), $n_{t h}^{i}$ can be either positive or negative. Of course, only positive $n_{t h}^{i}$ have physical sense. Correspondingly, figure 9 is plotted showing only positive $n_{t h}^{i}$. However, a helpful insight can be gained by looking at the neutral curve continuation behavior in the unphysical domain, as well. Figure 10 presents the same instability domain on the "extended" $n-\tau$ plane, i.e. with negative $n_{t h}^{i}$ included. The neutral curve segments $\left(\omega_{c}^{i} \tau=0, \omega_{c}^{i} \tau=\pi\right.$ and $\omega_{c}^{i} \tau=-\pi$ ) extend into the negative $n$-region and intersect with each other. These intersections are calculated numerically. Thus, positive $n_{t h}^{i}$ exists only in certain bands of $\tau$ (see appendix I for details). The figure shows links between the seemingly disjointed neutral curve segments presented in Figure 9.

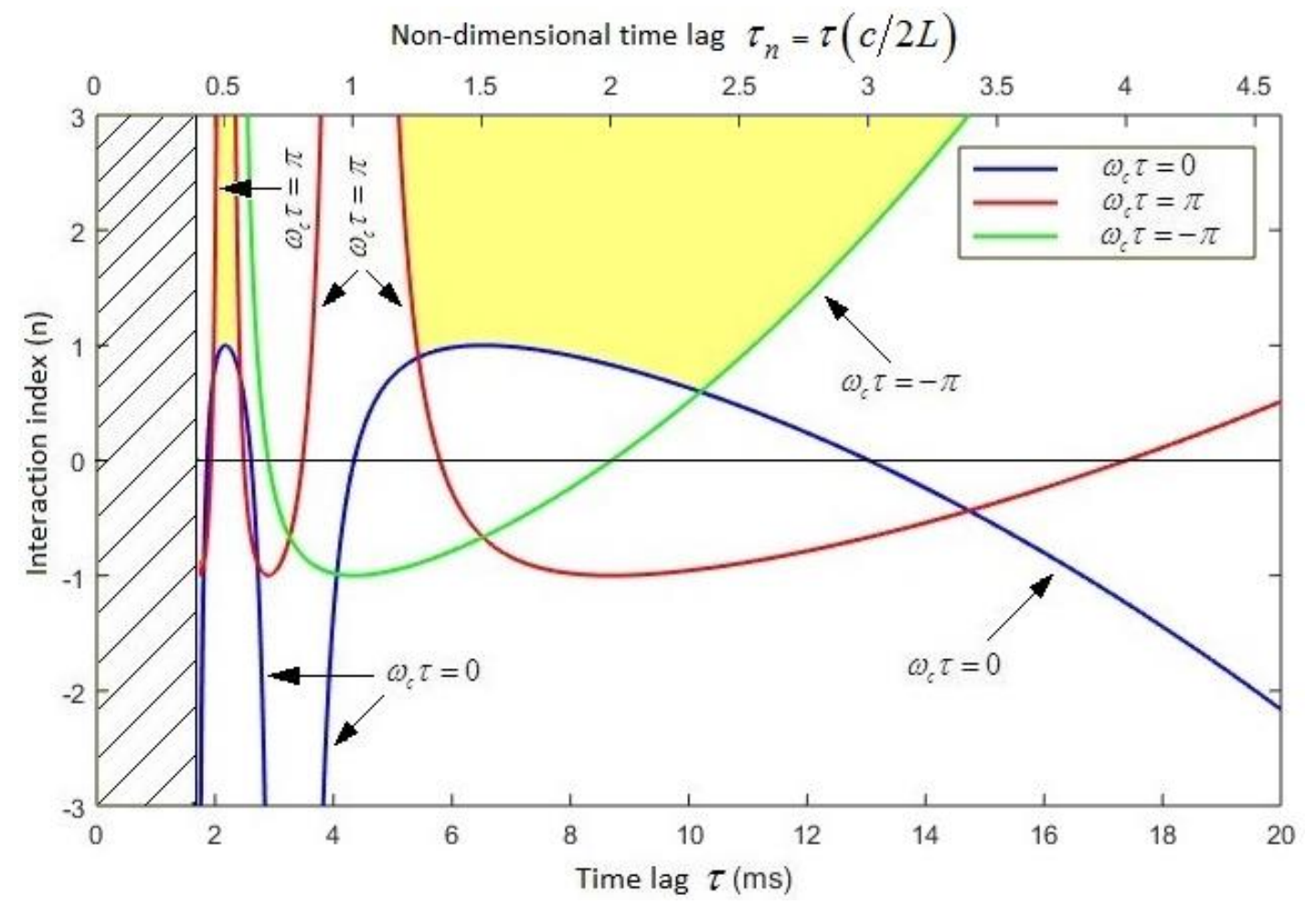

Figure 10: Neutral curve (26) and stability domain for the second intrinsic mode $\left(m^{i}=1\right)$ on the "extended" $n-\tau$ plane. The notation and parameters are the same as in figure 9. 


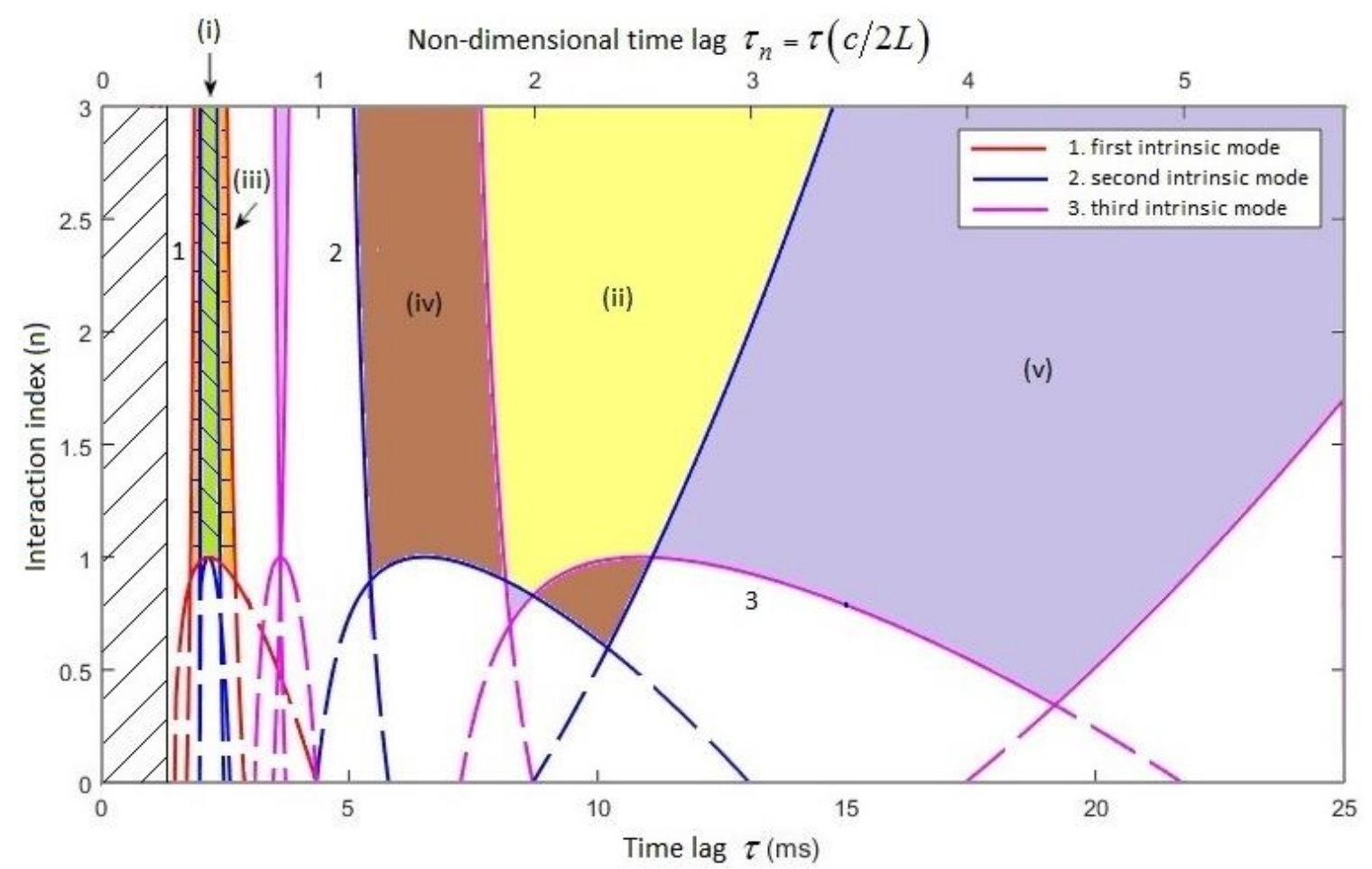

Figure 11: The neutral curves (26) and stability domain on the $n-\tau$ plane for the first $\left(m^{i}=0\right)$, second $\left(m^{i}=1\right)$ and third ( $\left.m^{i}=2\right)$ intrinsic modes, shown by numbers 1, 2 and 3 (Red, blue and magenta, respectively, online), when $x_{q}=L / 2$ and there is no temperature and cross-section jump. The common domain of instability for the first and second modes is marked in right hatching and indicated by (i) (lime online) and for the second and third intrinsic modes it is lightly shaded and indicated by (ii) (yellow online). Horizontal hatching indicated by (iii) (gold online), darker shaded domain indicated by (iv) (brown online) and medium density shaded indicated by (v) (lavender online) correspond to the non-overlapping domains of instability for the first, second and third intrinsic modes, respectively. Other parameters and notations are the same as in figure 9.

To give a better overall idea on the geometry of the instability domain with more than one mode we first consider just first three modes (that is for $m^{i}=0, m^{i}=1$ and $m^{i}=2$ ) simultaneously. Figure 11 illustrates the general tendencies: (i) as the mode number $m^{i}$ increases, the neutral curve loop shifts to the right; (ii) the span of the islands of instability on the left increases, as well; (iii) the segments corresponding to $\omega_{c}^{i} \tau=\pi$ and $\omega_{c}^{i} \tau=-\pi$ become less steep for higher modes. Note that for the first intrinsic mode $\left(m^{i}=0\right)$ the neutral curve segment $\omega_{c}^{i} \tau=-\pi$ is very peculiar. According to (26) $n_{t h}^{i}$ is infinity, while (19a) predicts zero frequency. First intrinsic mode, nonetheless, has neutral loop segments $\omega_{c}^{i} \tau=0$ and $\omega_{c}^{i} \tau=\pi$, which confine instability region that overlaps with the small neutral loops on the left for second intrinsic mode, as can be seen from figure 11 . 
Note that the characteristic values of $n_{t h}^{i}$ are smaller than to the threshold values of $n$ for an infinite tube found in [9]. For an infinite tube the same threshold in $n$ can be obtained by equating the growth/decay rate to zero in equation (19a). Hence we get, $n_{t h}^{i}=\left(S_{2} / S_{1}\right)\left(\rho_{1} / \rho_{2}\right)\left(c_{1} / c_{2}\right)+1$, which yields $n_{t h}^{i}=2$ for uniform tube, i.e., for $S_{2} / S_{1}=1$, $\rho_{1} / \rho_{2}=1$ and $c_{1} / c_{2}=1$. For a resonator $n_{\text {th }}^{i}$ can be substantially smaller, indeed. The range of values for $n_{t h}^{i}$, as shown in figures 9 and 11 for a quarter wave resonator lies in between 0.4 and 1. The observation that in a resonator the intrinsic modes can become unstable at a much lower value of $n$ might have major practical implications.

\subsubsection{Numerical validation of the analytical predictions for the neutral curve}

Here we will corroborate numerically analytical results of $\S 4.2 .1$ for $n_{t h}^{i}$, focusing on accuracy of the analytical predictions for the examples considered in figures 9 and 11. Figure 12 (a), (b) shows sample comparisons of the analytical prediction of $n_{\text {th }}^{i}$ (given by (26) and (25), respectively) with that of the exact numerical solution of (13) and (10), respectively for $m^{i}=1$. We neglect the effect of cross-section and temperature jumps in figure 12 (a), whereas, in figure 12 (b), we assume the following jumps: $S_{2} / S_{1}=1.5$ and $T_{2} / T_{1}=4$ (or, $\left.c_{2} / c_{1}=2\right)$ ). The flame is assumed to lie at $x_{q}=L / 2$ on both occasions. Dashed thin line (red online) shows the threshold for an infinite tube according to [9]. In our notations the expression for the threshold reads, $n_{t h}^{i}=\left(S_{2} / S_{1}\right)\left(\rho_{1} / \rho_{2}\right)\left(c_{1} / c_{2}\right)+1$. The plots 12 (a) and (b) demonstrate that the numerical solution corroborates very well the analytical prediction, whether the effect of cross-section and temperature jump is taken into consideration or not. To plot the exact numerical solution we manually track the loci of acoustic and intrinsic modes on the $n-\tau$ plane by gradually increasing $n$. In this way, we ascribe identities to the modes labeling them as intrinsic or acoustic. 


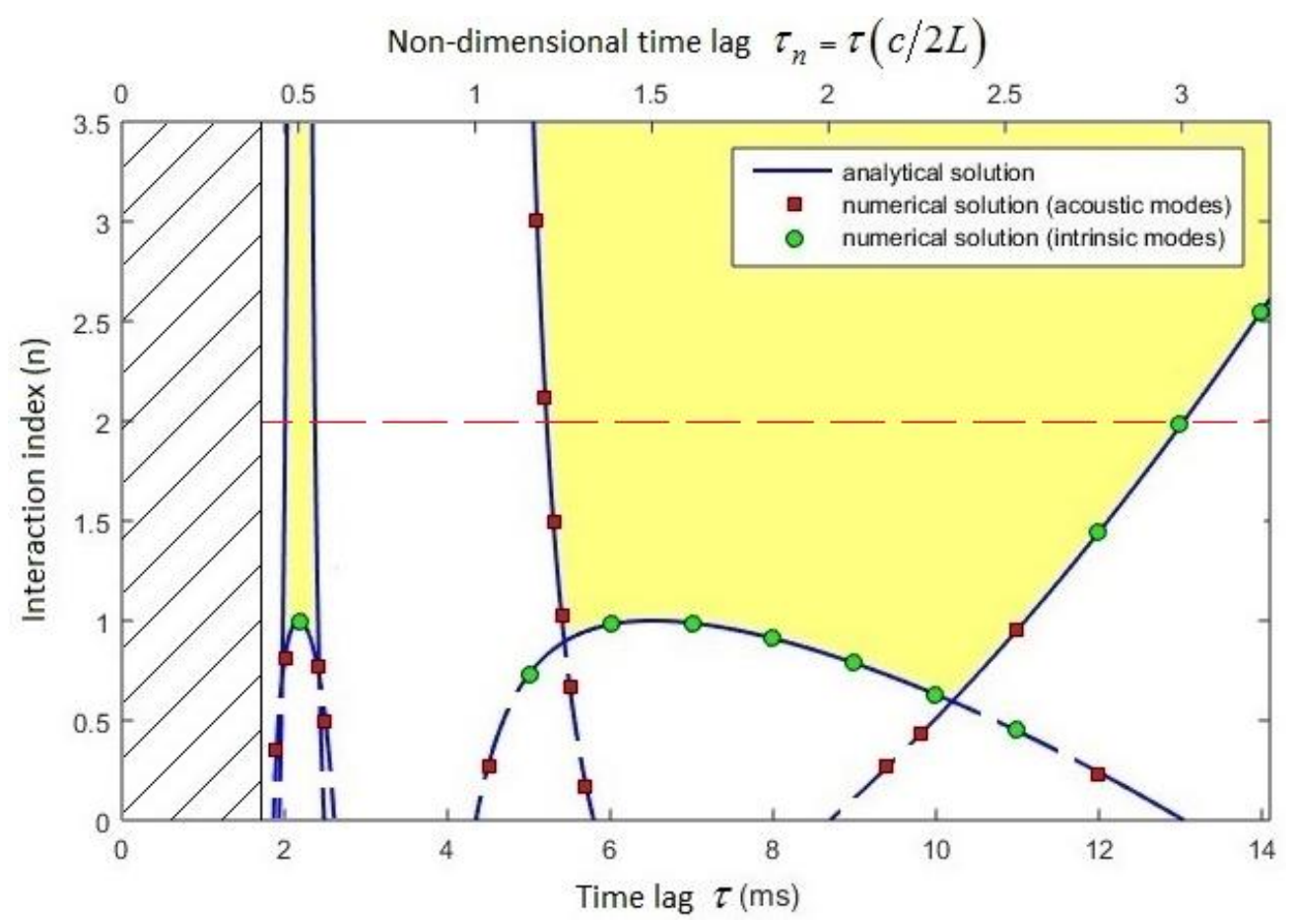

(a)

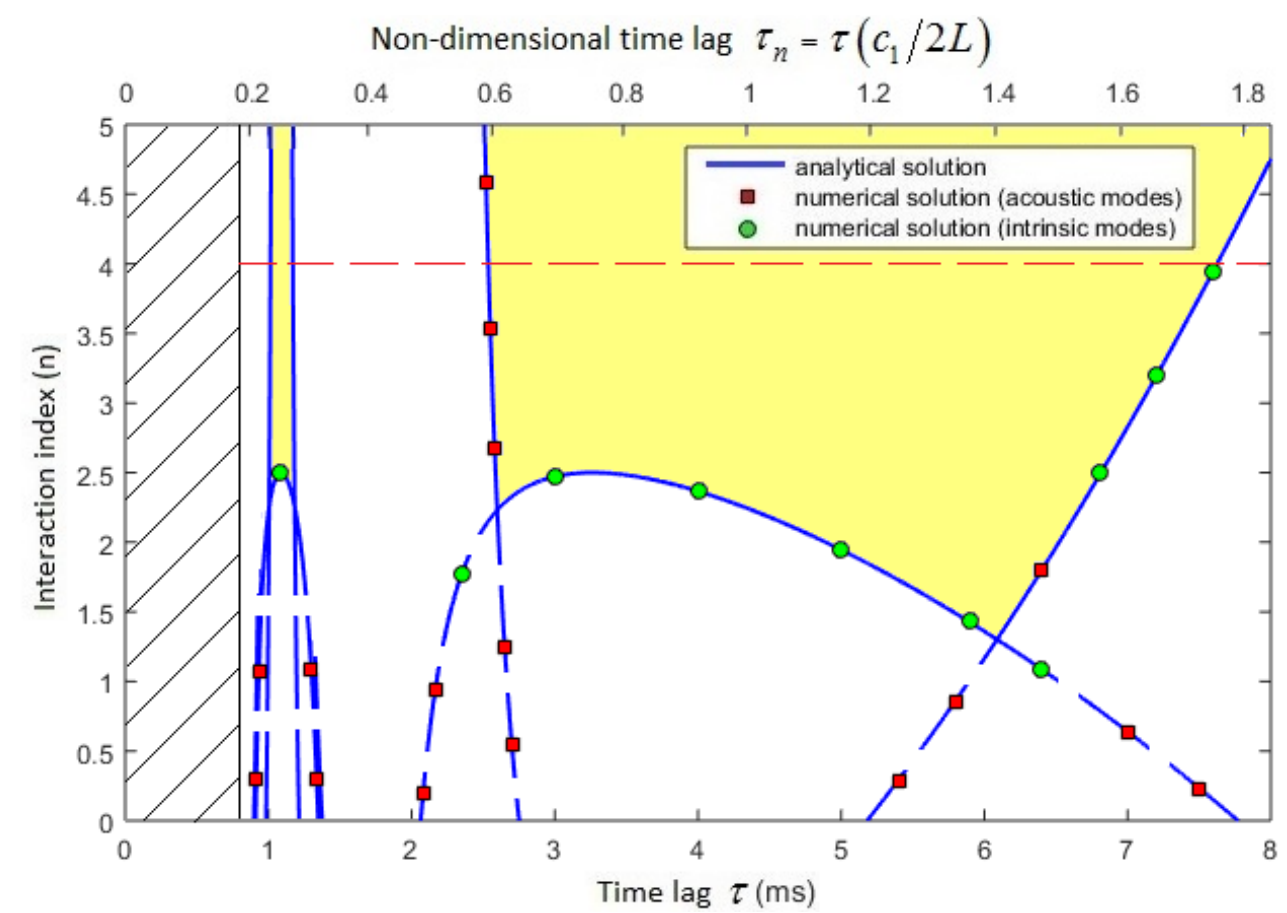

(b)

Figure 12: Neutral curve $(25,26)$ : comparison of analytical and numerical results for $m^{i}=1$, $x_{q}=L / 2$. (a) no cross-section jump or temperature jump, (b) $S_{2} / S_{1}=1.5$ and $T_{2} / T_{1}=4$ (or, $\left.c_{2} / c_{1}=2\right)$ ). Solid and dashed lines show the analytical solutions for intrinsic and acoustic modes, respectively. Numerical solutions are shown by circles (green online) and squares (red online). 
Dashed thin line (red online) shows the threshold for an infinite tube: $n_{\text {th }}^{i}=\left(S_{2} / S_{1}\right)\left(\rho_{1} / \rho_{2}\right)\left(c_{1} / c_{2}\right)+1$. Other parameters and notations are the same as in figure 9.

Figures 12 (a) and (b) also confirm the overall analytical picture. The instability domain has two distinctly different regions on the $n-\tau$ plane; the narrow islands of instability on the left and the main instability domain on the right. For the neutral curve on the right of figures 12 (a) and (b), the analytical prediction of $n_{t h}^{i}$ for the intrinsic modes, exactly coincide with the numerical solution. As mentioned in $\S 4.2 .1$, the combination of neutral curves $\omega_{c}^{i} \tau=0, \omega_{c}^{i} \tau=\pi$ and $\omega_{c}^{i} \tau=-\pi$, is indeed a repetitive pattern for any mode. Even though the numerical solution matches exactly the analytical prediction on the left side region of the $n-\tau$ plane as well, it is difficult to identify the nature of the modes in this region without an extra analysis. Also, near the intersection of neutral curve segments $\omega_{c}^{i} \tau=0, \omega_{c}^{i} \tau=\pi$, as well as, $\omega_{c}^{i} \tau=0, \omega_{c}^{i} \tau=-\pi$, intrinsic and coupled-acoustic modes invariably change their identities. Detailed discussion of these two aspects is beyond the scope of current paper. Further, a comparison of the neutral curves for the resonator and that of the infinite tube shows that the $n_{t h}^{i}$ is significantly lower for the resonator (see also $§ 4.2 .1$ ).

Thus, here we derived an explicit exact analytical expression (25) for the neutral curves on the $n-\tau$ plane. A set of neutral curves have been drawn for each mode. Figures 9 and 11 give a good idea of the geometry of the instability domains. The neutral curves have two qualitatively different regions. A region on the left, i.e. towards smaller values of $\tau$, comprises of loops with diminishing (towards smaller) $\tau$ widths, made of strongly coupled acoustic-intrinsic modes. On the other hand, the region on the right exhibits a single large loop. The neutral curve has three distinct segments corresponding to $\omega_{c}^{i} \tau=0, \omega_{c}^{i} \tau=\pi$ and $\omega_{c}^{i} \tau=-\pi$ in the $n-\tau$ space. The $\omega_{c}^{i} \tau=\pi$ and $\omega_{c}^{i} \tau=-\pi$ segments predict higher values of $n_{t h}^{i}$ compared to $\omega_{c}^{i} \tau=0$ segments. These segments are steep, but become less steep as the mode number, $m^{i}$ increases. For higher values of $m^{i}$, the span of the neutral curve segment $\omega_{c}^{i} \tau=0$ becomes wider and moves further to the right in the $n-\tau$ space. We can find analytically the loci of the intersection points of the neutral curve segments $\pm \omega_{c}^{i} \tau=\pi$ and $\omega_{c}^{i} \tau=0$. The analysis is, however, cumbersome and does not result in an easy to use formula. To get tractable formulae we look for asymptotic behavior for large and small $\tau$.

\subsubsection{Neutral curve asymptotics for small and large $\tau$}

Here we examine the neutral curve behavior for large and small $\tau$, which, in particular, will provide us compact formulae for $n_{t h}^{i}$ at the intersection points of neutral curve segments \pm $\omega_{c}^{i} \tau=\pi$, and $\omega_{c}^{i} \tau=0$ in the limit of large and small $\tau$. For simplicity, the effect of temperature and cross-section jump is neglected in the asymptotic analysis presented below. The flame is 
assumed to be at $x_{q}=L / 2$ and hence, the starting point of our analysis is equation (26). The analysis can be generalized to take into account temperature jump, cross-section jump and any flame location, as well. However, such a generalization goes beyond the scope of the present work.

\subsubsection{Asymptotics for small $\tau$}

It can be seen from figures 9 and 11 that the instability islands on the left hand side are quite narrow and the boundaries corresponding to $\omega_{c}^{i} \tau= \pm \pi$ are almost vertical. This trend continues further to the left as this pattern of neutral curves is repetitive. As these islands of instability become increasingly narrow, it becomes more difficult to resolve them accurately numerically. In this section, we make use of this narrowness of neutral curve loops and derive an approximate expression for the width of the instability domains bounded by these loops.

It can be shown (see appendix I, $\S$ AI.1), that the maximal value of $n_{t h}^{i}$ for the $\omega_{c}^{i} \tau=0$ segments of neutral curve is 1 (when, $S_{2} / S_{1}=1, c_{1} / c_{2}=1, \rho_{1} / \rho_{2}=1$ and $x_{q}=L / 2$ ) and we will use this to find $\tau$ corresponding to maximum $n_{\text {th }}^{i}$ for $\omega_{c}^{i} \tau=0$. Moreover, we can find the half width of the neutral loop for small $\tau$ regime, by estimating the distance (in terms of $\tau$ ) between the position of the local maximum of $n_{t h}^{i}$ and the intersection points of $\omega_{c}^{i} \tau=0$ and $\omega_{c}^{i} \tau= \pm \pi$. Here we will not seek exact analytical expressions for the intersection points of the segments $\omega_{c}^{i} \tau=0$ and $\omega_{c}^{i} \tau= \pm \pi$. Instead, assuming the neutral curve segments $\omega_{c}^{i} \tau= \pm \pi$ to be vertical and the intersection point of $\omega_{c}^{i} \tau=0$ and $\omega_{c}^{i} \tau= \pm \pi$ to correspond to $n_{t h}^{i}=1$, we can derive an analytical estimate for the half width of the neutral loop. Since the neutral curve segments $\omega_{c}^{i} \tau= \pm \pi$ are assumed to be vertical, the neutral curve loop width is independent of the $n_{t h}^{i}$ on $\omega_{c}^{i} \tau= \pm \pi$ segments.

First we find the location of $\tau$ corresponding to $n_{\text {th }}^{i}=1$ on the neutral curve segment $\omega_{c}^{i} \tau=0$. Let us call it $\tau_{1}$. Requiring $n_{t h}^{i}=1$, we immediately get from equation (26)

$$
\cos \lambda=-1 \text {, }
$$

where $\lambda=\left(2 m^{i}+1\right) \pi L / \tau_{1} c$. The solution of $(28)$ is

$$
\lambda=(2 m+1) \pi, m=0,1,2, \ldots
$$

where $m$ is the loop number of the neutral curve segment $\omega_{c}^{i} \tau=0$, with $m=0$, being the rightmost loop. As we show in appendix I, $m$ has to be restricted to odd integers only, i.e., $m=2 j+1$, because only for these loops $n_{t h}^{i}$ is positive, whereas, for the loops corresponding to even integers $m=2 j, n_{t h}^{i}$ is negative and, hence, these intersection points should be discarded 
from consideration. Making use of (29), we arrive at a simple expression for $\tau_{1}$ corresponding to the local maximum of $n_{t h}^{i}$ on $\omega_{c}^{i} \tau=0$,

$$
\tau_{1}=\left\{\left(2 m^{i}+1\right) /(2 m+1)\right\}(L / c) .
$$

We introduce $\tau_{2(+\pi)}$ and $\tau_{2(-\pi)}$ as the values of $\tau$ at the intersections of $n_{\text {th }}^{i}=1$ with the $\omega_{c}^{i} \tau= \pm \pi$ segments of the neutral curve. From (26) we find,

$$
\cos \lambda=1 / 3 \text {. }
$$

Solving (31) yields,

$$
\lambda=(2 m+0.392) \pi
$$

Then for $\omega_{c}^{i} \tau=\pi$ and $\omega_{c}^{i} \tau=-\pi$, we have $\lambda_{(+\pi)}=\left(2 m^{i}+2\right) \pi L / \tau_{2(+\pi)} c \quad$ and $\lambda_{(-\pi)}=\left(2 m^{i}\right) \pi L / \tau_{2(-\pi)} c$. Using (32) we find the points of the segment intersections, $\tau_{2(+\pi)}$ and $\tau_{2(-\pi)}$

$$
\tau_{2(+\pi)}=(L / c)\left\{\left(2 m^{i}+2\right) /(2 m+0.392)\right\} \text { and } \tau_{2(-\pi)}=(L / c)\left\{2 m^{i} /(2 m+0.392)\right\} .(33 \mathrm{a}, \mathrm{b})
$$

The distance between $\tau_{1}$ specified by (30) and $\tau_{2}$ given by (33 a, b) is the half width of the neutral loop in the asymptotic limit of small $\tau, \Delta \tau_{( \pm \pi)}=\left|\tau_{2( \pm \pi)}-\tau_{1}\right|$. On making use of (33a) and (33b) the solution for $\Delta \tau_{( \pm \pi)}$ reads,

$$
\Delta \tau_{(+\pi)}=\frac{L}{c}\left|\frac{\left(2 m+1.216 m^{i}+1.608\right)}{(2 m+1)(2 m+0.392)}\right| \text { and } \Delta \tau_{(-\pi)}=\frac{L}{c}\left|\frac{\left(2 m-1.216 m^{i}+0.392\right)}{(2 m+1)(2 m+0.392)}\right| \text {. }
$$

An equivalent non-dimensional version based on $\tau_{n}=\tau c / 2 L$ can be expressed as,

$$
\Delta \tau_{n,(+\pi)}=\frac{1}{2}\left|\frac{\left(2 m+1.216 m^{i}+1.608\right)}{(2 m+1)(2 m+0.392)}\right| \text { and } \Delta \tau_{n,(-\pi)}=\frac{1}{2}\left|\frac{\left(2 m-1.216 m^{i}+0.392\right)}{(2 m+1)(2 m+0.392)}\right| .
$$

This expression $(34 a, b)$ can be further simplified for higher values of $m$, $\Delta \tau_{( \pm \pi)} \approx(L / c)(1 / 2 m)$, which gives us the half width of the neutral loop for small $\tau$ and large $m$. Recall, that the central point of the neutral loop is given by (30).

Thus, we derived a simple expression for the neutral loop width valid for large $m$ and small $\tau$. The loop width is independent of the mode number of the intrinsic mode and is inversely proportional to the loop number $m$. This quantifies how the instability island width tends to zero as $\tau \rightarrow 0$. When $\tau \rightarrow 0$, the density of the loops increases and therefore, it would be close to impossible to capture faithfully this behavior using numerics. 


\subsubsection{Asymptotics for large $\tau$}

As figure 11 suggests, the largest neutral curve loop shifts towards larger values of $\tau$, with increase of the mode number. In this section we will find asymptotics of $n_{\text {th }}^{i}$ for large time lag $\tau$, that is $\tau>>\pi / c$ (see equation (AII.2) and the supporting discussions). Large $\tau$ assumption provides a way to find explicitly the intersection points for the neutral curve segments $\omega_{c}^{i} \tau=0$ and $\omega_{c}^{i} \tau= \pm \pi$ belonging to the largest loop, as shown in figure 9. At the intersection $n_{t h}^{i}$ simultaneously satisfies equations for the neutral curve segments $\omega_{c}^{i} \tau=0$ and $\omega_{c}^{i} \tau= \pm \pi$ given by (26).

Although, the range of $\tau$ for which this large $\tau$ analysis is valid might be too high from a practical combustor perspective, it is important to have an overall picture of the stability domain. The analysis addresses this need. Large $\tau$ part of the domain is also interesting because the lowest threshold and the highest growth rates occur there, which might serve as upper bounds for key characteristics of practical combustors. We also mention that as we show below simple formulae obtained using large $\tau$ asymptotics work remarkably well far beyond the domain their formal applicability.

The analysis (carried out in Appendix II) yields an explicit expression for the normalized time lag, $\tau_{n}\left(\tau_{n}=\tau c / 2 L\right)$, written as $\tau_{n(+\pi, 0)}$, to stress the fact that it corresponds to the intersection point of $\omega_{c}^{i} \tau=0$ and $\omega_{c}^{i} \tau=\pi$, valid for large $\tau$,

$$
\tau_{n(+\pi, 0)}=\left(4 m^{i}+3\right) /\left\{2\left(2 m_{(+\pi, 0)}+1\right)\right\} .
$$

Here, $m_{(+\pi, 0)}=0,1,2, \ldots m_{(+\pi, 0)}$, is the intersection point number of the neutral curve segments $\omega_{c}^{i} \tau=0$ and $\omega_{c}^{i} \tau=\pi$, with $m_{(+\pi, 0)}=0$ being the rightmost intersection point, while $m^{i}$ is the mode number. Similar consideration of the intersections of the neutral curve segments $\omega_{c}^{i} \tau=0$ and $\omega_{c}^{i} \tau=-\pi$ yields,

$$
\tau_{n(-\pi, 0)}=\left(4 m^{i}+1\right) /\left\{2\left(2 m_{(-\pi, 0)}+1\right)\right\}
$$

Here $m_{(-\pi, 0)}$ is an integer indicating the intersection point number of the neutral curve segments $\omega_{c}^{i} \tau=0$ and $\omega_{c}^{i} \tau=-\pi$, with $m_{(-\pi, 0)}=0$ being the rightmost intersection point, while $m^{i}$ is the mode number. The $n_{\text {th }}^{i}$ corresponding to (35) and (36) can be given as,

$$
n_{t h(+\pi, 0)}^{i}=2 \sin \left(3 \pi / 8 m^{i}\right) /\left[\sin \left(3 \pi / 8 m^{i}\right)+1\right],
$$




$$
n_{t h(-\pi, 0)}^{i}=2 \sin \left(\pi / 8 m^{i}\right) /\left[\sin \left(\pi / 8 m^{i}\right)+1\right]
$$

This expression quantifies how $n_{t h(+\pi, 0)}^{i}, n_{t h(-\pi, 0)}^{i}$ tends to zero as $m^{i}$ increases. A comparison of (37) and (38) shows that $n_{t h(-\pi, 0)}^{i}<n_{t h(+\pi, 0)}^{i}$. Thus the intersection points of neutral curve segments $\omega_{c}^{i} \tau=0$ and $\omega_{c}^{i} \tau=-\pi$, correspond to lower value of $n_{\text {th }}^{i}$ compared to the intersection of neutral segments $\omega_{c}^{i} \tau=0$ and $\omega_{c}^{i} \tau=\pi$.

Thus, to avoid dealing with large $m_{(+\pi, 0)}, m_{(-\pi, 0)}$ and large $\tau$ regimes head on, we found simple analytical description of the points of intersection of the segments of neutral curves $\omega_{c}^{i} \tau=0$ and $\omega_{c}^{i} \tau=\pi$, as well as, $\omega_{c}^{i} \tau=0$ and $\omega_{c}^{i} \tau=-\pi$, assuming large $\tau$. At the intersection of neutral curve segments, $n_{t h}^{i}$ decreases with increase of mode number. $n_{t h}^{i}$ at the intersection of $\omega_{c}^{i} \tau=0$ and $\omega_{c}^{i} \tau=\pi$ is always larger than $n_{\text {th }}^{i}$ at the intersection of $\omega_{c}^{i} \tau=0$ and $\omega_{c}^{i} \tau=-\pi$. The extreme right intersection point of $\omega_{c}^{i} \tau=0$ and $\omega_{c}^{i} \tau=-\pi$ (that is, the intersection of the large loop with $m_{(-\pi, 0)}=0$ as given by (36)) corresponds to the lowest value of $n_{\text {th }}^{i}$ for any fixed mode number. These asymptotics prove to be extremely useful for finding the bounds of the instability domain in in $\S 4.4$ and estimates of the maximal growth rate in $\S 4.5$.

\subsection{The growth/decay rates near the neutral curve}

In the context of intrinsic mode instability, the growth rate is of prime interest. The derivation of growth/decay rate directly from the original dispersion relation (10) for all values of $n$ on the $n-\tau$ plane, is a very challenging, but hardly a priority task. For large deviations from the neutral curve, the mode in question is either strongly decaying and, hence, plays little role in the dynamics of the system, or has too high growth rates, which implies that the linear theory we adopted quickly ceases to be applicable. Thus, only the growth/decay rates near the neutral curve are of true interest.

\subsubsection{Analytical expression for the growth rate}

Thanks to the discovered phenomenon of decoupling on the neutral curve, in the previous section we established the exact location of the neutral curve on the $n-\tau$ plane. Here, exploiting the decoupling and proximity to the neutral curve, we will find analytically the growth/decay rate in the curve vicinity. To this end consider a point $(n, \tau)$ on the $n-\tau$ plane near the neutral curve for a particular mode $m^{i}$, denoting the deviation from the curve as $n_{1}^{i}$, i.e. $n-n_{t h}^{i}=n_{1}^{i}<<n_{t h}^{i}$. The eigen-frequency in the chosen point $(n, \tau)$ differs from its value on the neutral curve $\omega_{0}^{i}$ for the same $\tau$. We denote this deviation as $\omega_{1}^{i}$. Assume that the real part of $\omega_{1}^{i}$ is small and of little interest near the neutral curve. This implies that $\omega_{1}^{i}$ represents the growth/decay rate. A priori 
this assumption is difficult to justify rigorously. However, the fact that the real part of eigenfrequency on the $\omega_{c}^{i} \tau=0$ segments of the neutral curve is exactly the same as for the limit of small $n$ suggests that $\omega_{1}^{i}$ has negligibly small real part. This assumption, as we show below, is also supported by numerics.

Hence, $n=n_{t h}^{i}+n_{1}^{i}, \omega=\omega_{0}^{i}+\omega_{1}^{i}$. Substituting this expansion of $n$ and $\omega$ into the original dispersion relation (10), neglecting higher order terms in $\omega_{1}^{i}$ in sin and cos function expansion and subtracting the original dispersion relation (10) for $\omega=\omega_{0}^{i}$ from this form, we get a perturbed form of the dispersion relation (10), which yields an explicit analytical expression for the growth/decay rate $\omega_{1}^{i}$,

$$
\omega_{1}^{i}=\frac{2 n_{1}^{i} e^{i \omega_{0}^{i} \tau} \sin \left(\omega_{0}^{i} x_{q} / c_{1}\right) \sin \left(\omega_{0}^{i}\left(x_{q}-L\right) / c_{2}\right)}{\left[(\alpha+1) \beta_{1} \sin \left(\omega_{0}^{i} \beta_{1}\right)+(\alpha-1) \beta_{2} \sin \left(\omega_{0}^{i} \beta_{2}\right)-\Sigma\right]} .
$$

where,

$$
\Sigma=2 n_{t h}^{i} e^{i \omega_{0}^{i} \tau}\left\{\begin{array}{c}
i \tau \sin \left(\omega_{0}^{i} x_{q} / c_{1}\right) \sin \left(\omega_{0}^{i}\left(x_{q}-L\right) / c_{2}\right)+\left(x_{q} / c_{1}\right) \cos \left(\omega_{0}^{i} x_{q} / c_{1}\right) \sin \left(\omega_{0}^{i}\left(x_{q}-L\right) / c_{2}\right) \\
+\left(\left(x_{q}-L\right) / c_{2}\right) \sin \left(\omega_{0}^{i} x_{q} / c_{1}\right) \cos \left(\omega_{0}^{i}\left(x_{q}-L\right) / c_{2}\right)
\end{array}\right\}
$$

while $\alpha, \beta_{1}$ and $\beta_{2}$ are given by (15). It can be verified by direct calculation of $\omega_{1}^{i}$, that its real part is indeed insignificant, as was assumed. Thus, $\omega_{1}^{i} \approx \operatorname{Im}\left(\omega_{1}^{i}\right)$ and will be treated as growth/decay rate. We stress that in contrast to the frequencies on the neutral curve, according to (39) $\omega_{1}^{i}$ depends on all combustor parameters, including the temperature and cross-section jump and the flame location.

Thus, we have derived a general explicit expression for the intrinsic mode growth/decay rates in the vicinity of the neutral curve on the $n-\tau$ plane. For simplicity, only the first order term in $n_{1}^{i}$ has been presented. Higher order corrections in $n_{1}^{i}$ could be found in a similar fashion. The justification of our a priori assumption and the accuracy of the analytical results will be demonstrated by comparison with the numerical solution in $\S$ 4.3.2. The formula above gives the growth/decay rates for each intrinsic mode. In principle, by considering all the modes we can find the absolute maximal growth rate or the least decay rate for any point $(n, \tau)$ on the $n-\tau$ plane. In practice, it might be difficult to deal with an infinite number of modes and therefore, alternatives are needed.

\subsubsection{Numerical validation of analytical expression}

Here we validate the analytical growth rate given by (39) with numerical solution of (10) and (13). The comparison will also give us an idea of the range of applicability and accuracy of our analytical approach. Figure 13 (a) presents results for the case of uniform temperature and cross- 
section, whereas, figure 13 (b) shows comparison for the combustor with a temperature and cross-section jump across the flame.

Figure 13 (a) shows a comparison of the analytical prediction of the growth rates given by (39) with that of the numerical results. In the vicinity of $n_{\text {th }}^{i}$ (0.98 for this case), analytical and numerical predictions agree quite well. However, as we move away from $n_{t h}^{i}$ the analytical prediction starts to deviate from the numerical results. As evident from figure 13 (b), this observation holds if the effect of temperature jump is also taken into account. We can, therefore, conclude that the analytical prediction for the growth rate is in good agreement with the numerical solution in the vicinity of $n_{t h}^{i}$, where it is of most interest. Expectedly, as we move away from the vicinity of $n_{t h}^{i}$, the accuracy of approximate solution (39) decreases. However, we stress that a massive twenty percent deviation from $n_{\text {th }}^{i}$ results only in smaller than two percent discrepancy of the growth/decay rate. Recall also that our approximate solution (39) is a just the leading order term of expansion in $n_{1}^{i}$ (the departure of $n$ from $n_{t h}^{i}$ ). Taking into account the next terms of the expansion would have further improved the accuracy. However, we do not pursue this line here.

Although, here we present just two examples of validation of our prediction of the growth rate, we stress that it has been thoroughly checked that the analytical prediction of the growth rate (39) works very well for all $\tau$, including the vicinity of intersections of different segments of the neutral curve. Growth rate formula (39) works equally well when the effects of cross-section and temperature jump are taken into consideration.

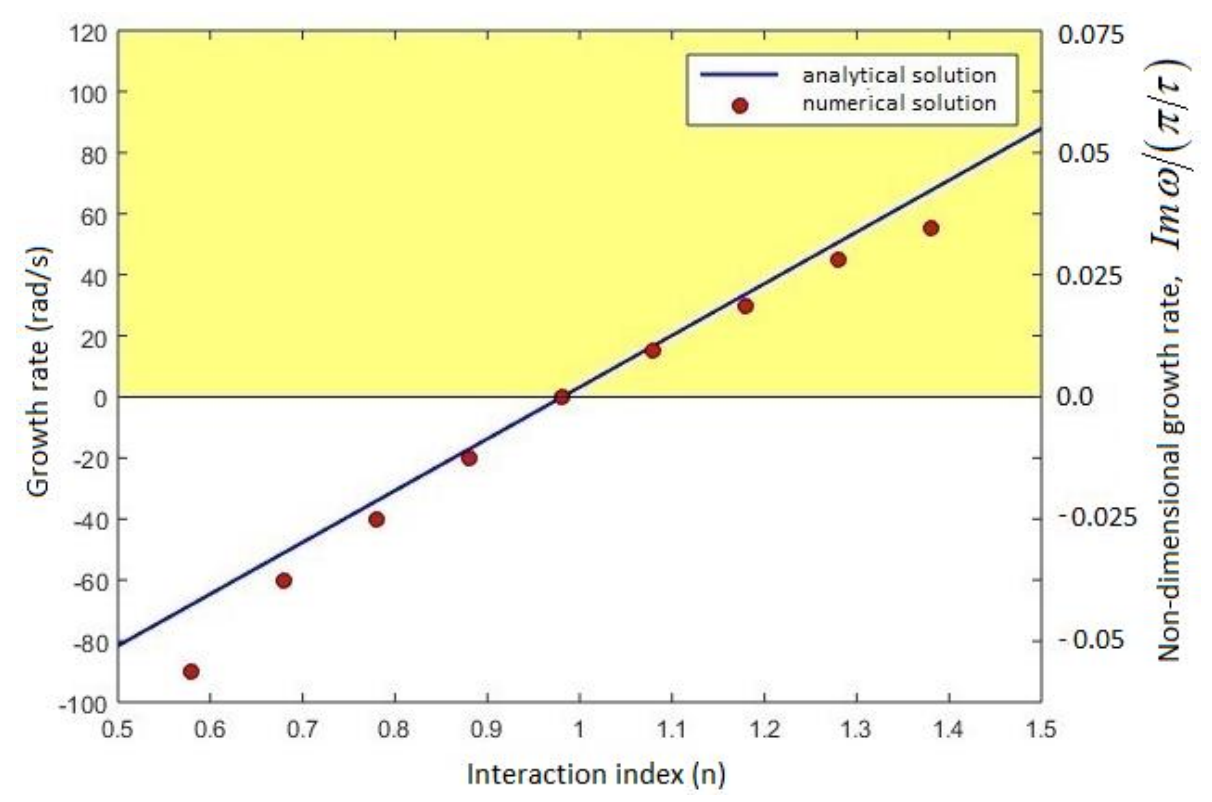

(a) 


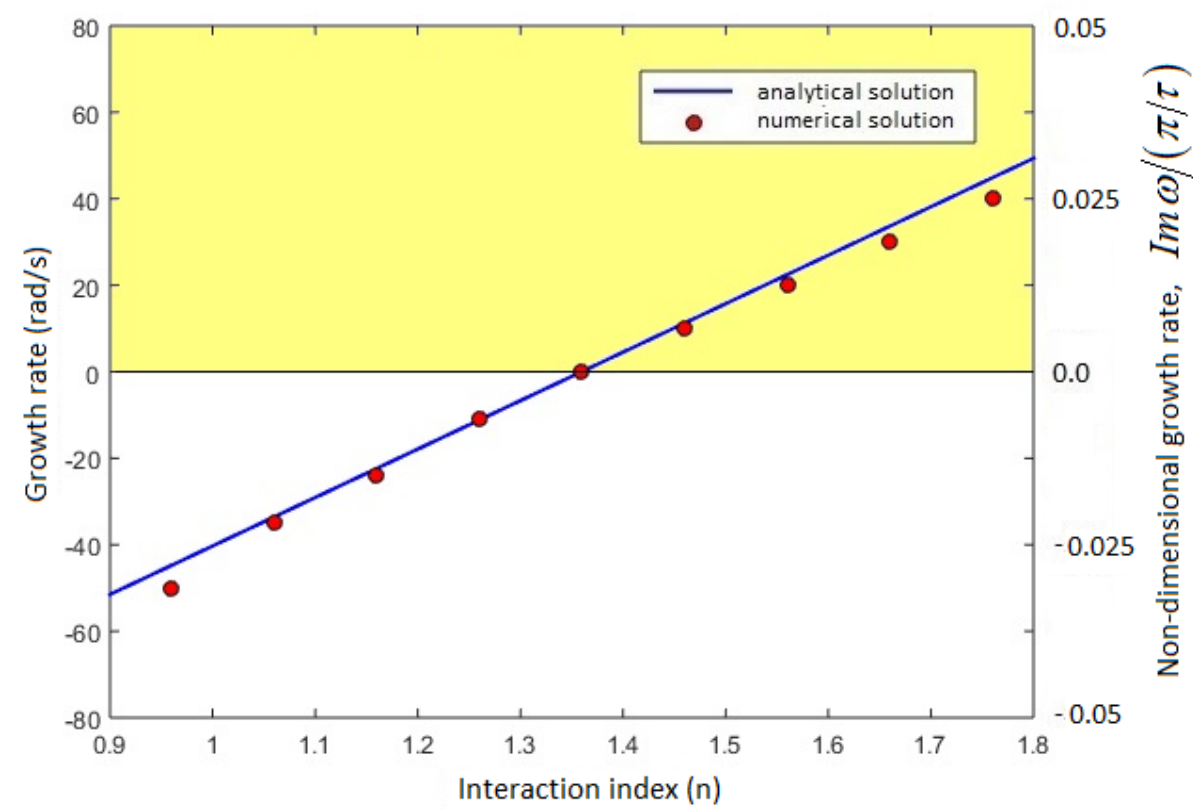

(b)

Figure 13: Growth rate for $m^{i}=1$ at $\tau=6 \mathrm{~ms}, x_{q}=L / 2$ : (a) comparison of analytical result (39)

$$
\text { and numerical solution of the dispersion relation (13), when } S_{2} / S_{1}=1 \text { and } T_{2} / T_{1}=1 \text {; (b) }
$$
comparison of analytical result (39) and numerical solution of the dispersion relation (10), when $S_{2} / S_{1}=1.5$ and $T_{2} / T_{1}=4$ (or, $\left.c_{2} / c_{1}=2\right)$ ). Solid line and circles show the analytical and numerical solutions, respectively. Dimensional ( $\mathrm{rad} / \mathrm{s}$ ) and non-dimensional (growth rate is normalized by the intrinsic instability frequency - $1570 \mathrm{rad} / \mathrm{s}$ ) scales are used for the growth rate. The instability domain is lightly shaded (yellow online). Other parameters are the same as in Fig.9.

\subsection{Bounds on the stability domain}

In $\S 4.3$ we mentioned a potential difficulty in finding the boundary of stability domain and growth/decay rates for any point on the $n-\tau$ plane, when all the modes are taken into account. Indeed, the small loops, on the left of the neutral curves for higher modes, overlap with the large loop of the neutral curves for lower modes, which creates an extremely intricate picture of overlapping loops of different, often incomparable, widths for various modes. So, here we suggest an alternative or a "short-cut". Instead of dealing with intricately curved stability domain specified by contributions of infinite number of modes, we coarse-grain the stability behavior of all the intrinsic modes and develop an "integral" stability map that provides an upper and lower bound of the stability domain on the $n-\tau$ plane. Figure 14 introduces the concept of a stability envelope for the intrinsic modes. For the sake of simplicity only the intrinsic modes one to six are shown in the figure. The effect of cross-section and temperature jumps across flame is neglected here, even though it is straightforward to extend the analysis for generalized case with temperature and cross-section jump. Only the outermost stability loops for each mode are plotted; as it has been discussed in the previous section that the outermost stability loops correspond to the minimal value of $n_{t h}^{i}$. The dash dotted black line indicates the upper bound of 
the stability envelope and the dashed black line shows the lower bound of the stability envelope. Short dashed thin line (pink online) shows the analytical prediction (38) of the lower bound. Thus, there exists three distinctly different regions of the coarse-grain stability:

i. For any point on the $n-\tau$ plane below the lower bound of the stability envelope, all the intrinsic modes are linearly stable.

ii. For any point on the $n-\tau$ plane above the upper bound of the stability envelope, all the intrinsic modes are linearly unstable.

iii. For any point on the $n-\tau$ plane in between the upper and lower bound of the stability envelope, the intrinsic modes can be linearly stable (for $n-\tau$ values falling into the white region) or unstable (for $n-\tau$ values corresponding to the lightly shaded region (marked in yellow online)). For the points lying in the lightly shaded (yellow online) region within the stability envelope, roughly, the maximal growth rate is proportional to the vertical distance of the point from the lower bound of the stability envelope (this distance can be interpreted as $n_{1}^{i}$ in (39)). Similarly, for the points lying in the white region above the lower stability envelope, the maximal decay rate is proportional to the vertical distance of the point from the upper bound of the stability envelope. This domain needs extra analysis, which is carried out in the next section.

The point, we want to re-iterate here, is that the large $\tau$ asymptotics formulae $(36,38)$ for the lower bound of the stability envelope provides a good accuracy even far beyond the range of its formal validity.

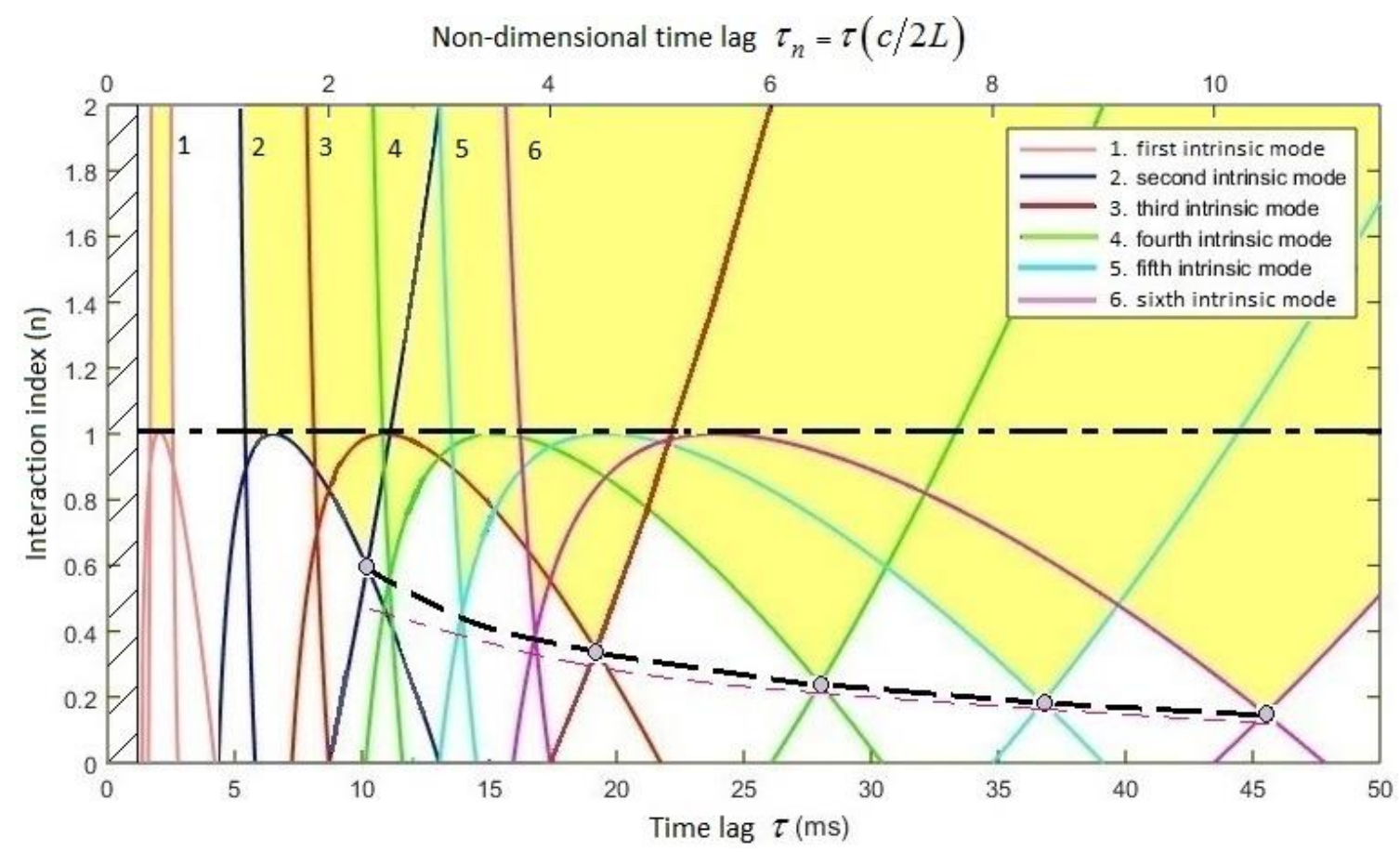

Figure 14: Neutral curve (26) and stability domain on the $n-\tau$ plane for the first six intrinsic modes, when $x_{q}=L / 2$ and there is no temperature and cross-section jump. The instability 
domain is lightly shaded (marked in yellow online). Black dashed line connecting intersections indicated by circles shows the instability domain lower envelope; that is the locus of the lowest threshold of instability for each mode. Below this envelope, all points on the $n-\tau$ plane are guaranteed to be stable. Short dashed thin line (pink online) shows the analytical prediction (38) of the lower bound. Black dash-dotted line is the locus of the maximal threshold of instability for each mode. Above this line all points on the $n-\tau$ plane are unstable. Between the dash-dotted and the dashed line, there is conditional instability domain. Stability at any point $(n, \tau)$ lying between these two lines depends on whether the point is in white or lightly shaded (yellow online) region. Hatched area indicates the domain with multiple instability islands of decreasing

scale, which are not shown. Also not shown are the narrow loops due to higher modes. For simplicity only the big right loop of neutral curves for each mode were plotted, since these loops provide the minima of $n_{t h}^{i}$. Other parameters used are the same as in Fig.9.

\subsection{Estimates of the maximal growth rates}

In itself the distance to the bounds of the stability envelope can provide only qualitative tendencies of intrinsic mode growth/decay rates as function of the parameters of the system. To quantify the growth/decay rates we need to know the mode number of the dominant mode. To this end we can employ the large $\tau$ asympotics (35-38), which were found to work surprisingly well for the whole range of $\tau$ exceeding $\tau=\pi L / c$ (see appendix II). In the generic case, any given $\tau$ specifies the two nearest mode numbers (see Fig AII.1a and the follow up discussion). As can be seen from figures 11 and 14, all modes with numbers below those of these two modes are stable (for large $n$ ) for this $\tau$ and thus, their growth rates need not be considered. Then the maximal growth rate can be easily estimated using (39), just by applying it to these two modes. It can be shown that all the higher modes have smaller growth rates and, therefore, can be ignored.

We elaborate this point by extra figure 15. Similar to figure 14, effect of cross-section and temperature jumps across flame is neglected here, as well. Let us consider stability at a sample point on the $n-\tau$ plane, choosing, say, $n=0.9$ and $\tau=16.5 \mathrm{~ms}$ (the point is indicated by the diamond). On the $n-\tau$ plane, according to our large $\tau$ asymptotics (36), the chosen value of $\tau$, $\tau=16.5 \mathrm{~ms}$ falls somewhere in between the values of $\tau$ corresponding to the minima of $n_{\text {th }}^{i}$ for the second and third modes. Hence, figure 15 depicts just these two intrinsic modes in form of their large loops of the neutral curve. As has been found in $\S 4.2 .3 .2, n_{\text {th }}^{i}$ is minimal for the extreme right intersection point of $\omega_{c}^{i} \tau=0$ and $\omega_{c}^{i} \tau=-\pi$. Thus, the large neutral curve loop on the right correspond to the dominant intrinsic mode instability. Any instability corresponding to the smaller loops of overlapping higher order modes will be weaker. Hence, the maximal growth rate will be determined by proximity to the large neutral curve loop on the right. This will form the basis of our maximum growth rate estimate at a particular point on the $n-\tau$ plane. The dashed vertical line (purple online) represents the formal (optimistically interpreted) threshold of applicability of the large $\tau$ asymptotics, $\tau=\pi L / c$ (see (AII.2) and the supporting discussion). 
As we showed, the large $\tau$ asymptotics can be used with high confidence for all $\tau$ exceeding this threshold. For the particular example indicated by diamond ( $n=0.9, \tau=16.5 \mathrm{~ms}$ ) the second intrinsic mode according to (39) has a negative growth rate, while the actual growth rate of the third intrinsic mode is $23 \mathrm{rad} / \mathrm{s}$. Thus, for any $\tau$ exceeding $\tau=\pi L / c$, we can pinpoint the particular mode which has the highest growth rate and find using explicit expression (39). It has been checked by direct calculation that all higher intrinsic modes either have smaller growth rates than the third intrinsic mode or remain stable. Note that had we taken the distance (along the vertical) between the diamond and the dashed line (green online) in figure 15 as an estimate of $n_{1}^{i}$, then the maximal growth rate would have been $69 \mathrm{rad} / \mathrm{s}$ (assuming $n_{t h}^{i} \approx 0.4$ in (39)).

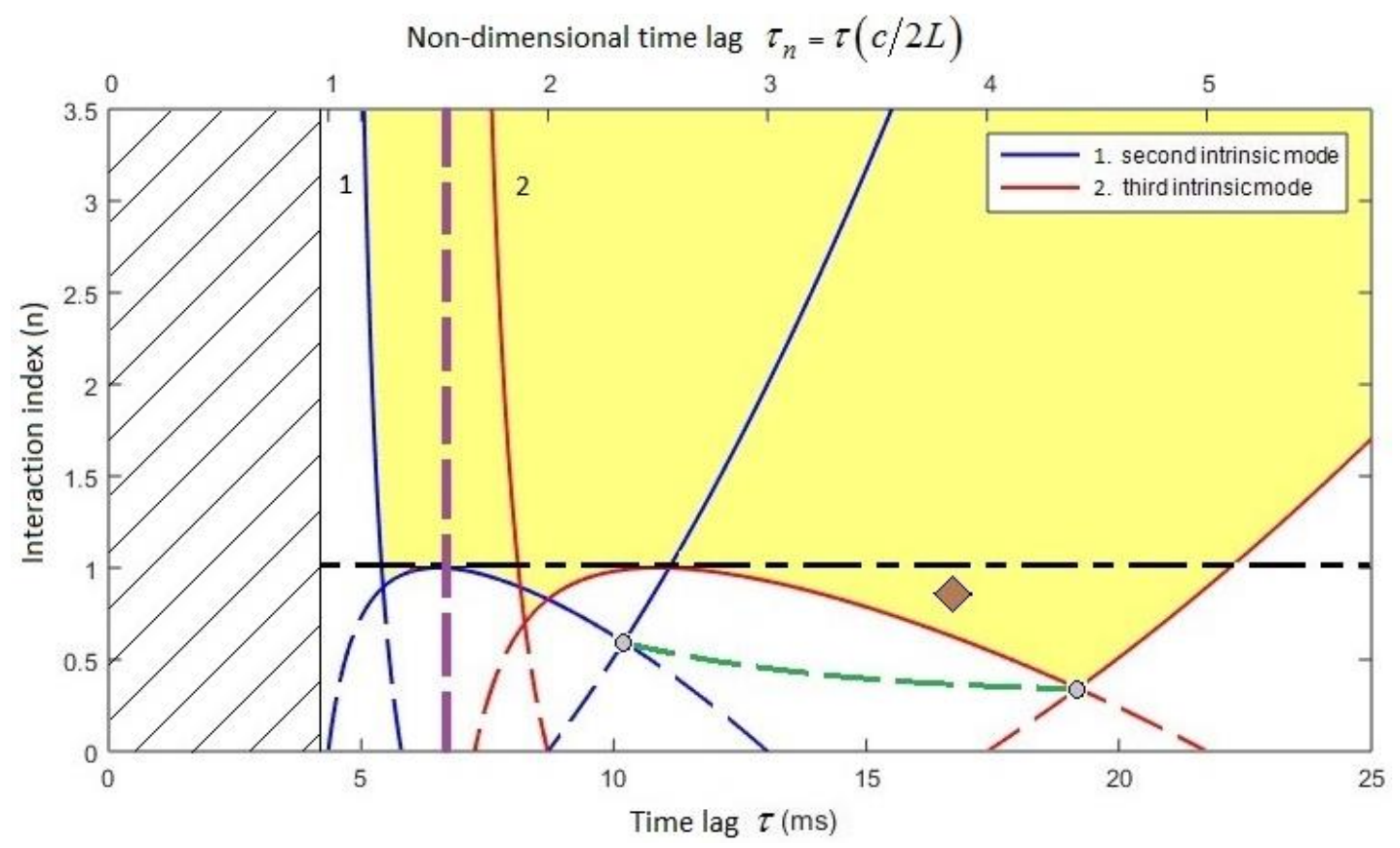

Figure 15: Neutral curve (26) and stability domain on the $n-\tau$ plane for two intrinsic modes $m^{i}=1,2$, when $x_{q}=L / 2$ and there is no temperature and cross-section jump. This figure shows a subset of curves from figure 14. Note that the lower bound of instability is shown by a dashed line (green online). The diamond indicates the chosen sample point $n=0.9$ and $\tau=16.5 \mathrm{~ms}$ on the $n-\tau$ plane. The dashed vertical line (purple online), $\tau=\pi L / c$, is the threshold in $\tau$ above which the large $\tau$ assumption applies (see (AII.2) and the supporting discussion). Other parameters used are the same as in Fig.9.

Thus, the coarse grained stability diagram is helpful for a rough instant stable/unstable answer. For a realistic estimate of the maximal growth rate or minimal decay rate one has to identify first the most unstable mode using either (36) or figure (AII.1a). 


\section{Dependence of intrinsic mode stability on the cross- section/temperature jumps and flame location}

Equation (19a) describes how the dispersion relation for strongly decaying intrinsic modes depends on the cross-section jump and temperature jump in the range of small $n$. In this section, we will find how $n_{t h}^{i}$ and growth rate depends on the main parameters of our combustor model: (i) cross-section jump, (ii) temperature jump and (iii) flame location.

Figure 16 illustrates the dependence of the neutral curve main loop on cross-section jump, plotted on the basis of (25). It presents two different cases of cross-section jump: $S_{2} / S_{1}=1$ and $S_{2} / S_{1}=2$. The effect of temperature jump across flame is neglected here and the flame is assumed to situate at $x_{q}=L / 2$. Darker shaded domain (brown online) and medium density shaded domain (lavender online) mark the instability domains for each case, with the common domain being lightly shaded (marked in yellow online). In all subsequent figures the common instability domain is also lightly shaded (marked in yellow online). It can be seen from figure 16 that the span of the largest instability domain on the right is wider for lower values of cross section jump, whereas, the peak $n_{\text {th }}^{i}$ remains the same.

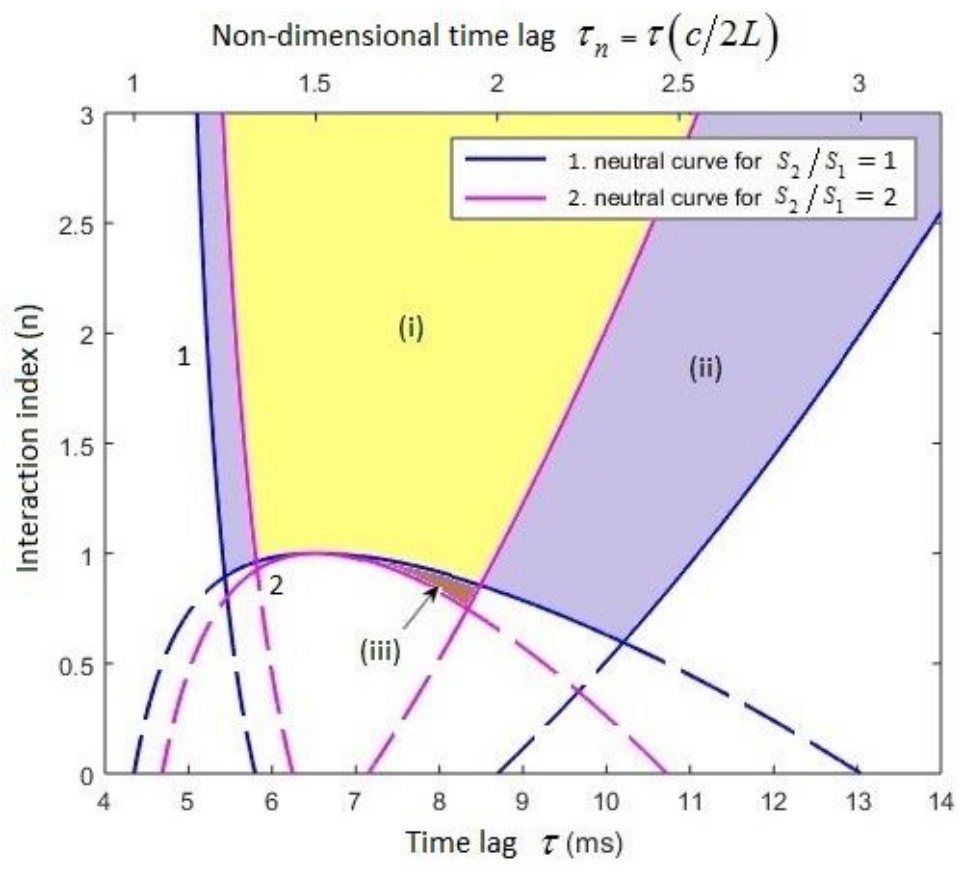

Figure 16: Effect of cross-section jump on the stability domain for $m^{i}=1, x_{q}=L / 2$ and no temperature jump. The plot is based on (25) and shows no cross-section jump $\left(S_{2} / S_{1}=1\right)$ and with $\left(S_{2} / S_{1}=2\right)$ cross section jump configurations. The instability domains for the cases $S_{2} / S_{1}$ $=1$ and $S_{2} / S_{1}=2$ are indicated by medium density shaded domain (ii) (lavender online) and darker shaded domain (iii) (brown online), respectively. The common instability domain for 
these two cases is lightly shaded and indicated by (i) (marked in yellow online). Other parameters and notations are the same as in Fig.9.

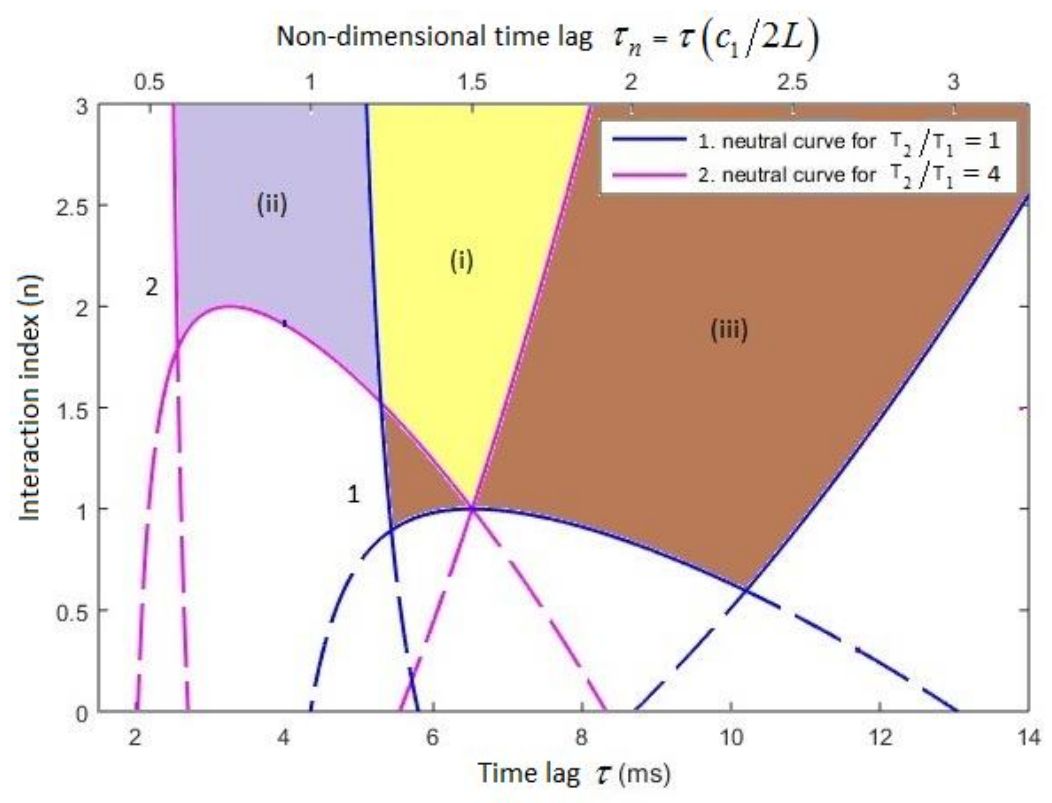

(a) $x_{q}=L / 2$

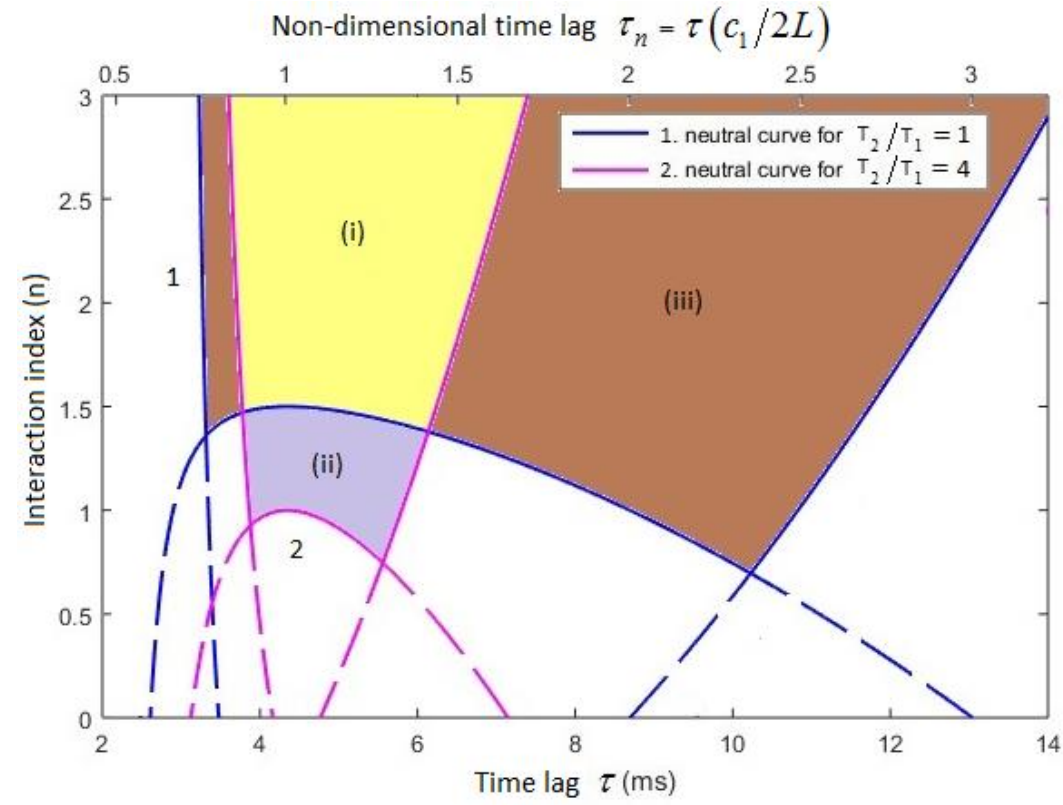

(b) $x_{q}=L / 3$

Figure 17: Effect of temperature jump on stability domain for $m^{i}=1$, with no cross-section jump. The plot is based on (25) and shows configurations with no temperature jump, i.e. $T_{2} / T_{1}$ $=1$ (or, $\left.c_{2} / c_{1}=1\right)$ ) and with $T_{2} / T_{1}=4$ (or, $\left.c_{2} / c_{1}=2\right)$ ) temperature jump. Figures (a) and (b) are for two flame locations $x_{q}=L / 2$ and $x_{q}=L / 3$. The instability domains for the cases $c_{2} / c_{1}=1$ and $c_{2} / c_{1}=2$ are indicated by darker shaded domain (iii) (brown online) and medium density 
shaded domain (ii) (lavender online), respectively. The common instability domain for these two cases is lightly shaded and indicated by (i) (marked in yellow online). Other parameters and notations are the same as in Fig.9.

The effect of temperature jump on $n_{t h}^{i}(\tau)$ is slightly different. Figure 17, plotted on the basis of (25), compares two different cases of temperature jump: for $T_{2} / T_{1}=1$ (or, $c_{2} / c_{1}=1$ ) and $T_{2} / T_{1}=4$ (or, $c_{2} / c_{1}=2$ ). These two different cases are shown for the flame locations (a) $x_{q}=L / 2$ $(=0.375 \mathrm{~m})$ and (b) $x_{q}=L / 3(0.25 \mathrm{~m})$, respectively. The effect of cross-section jump across flame is neglected here. Figure 17 (a) shows that temperature jump stretches the neutral curve main loop slightly to the left but, at the same time, the span reduces on the right hand side. The peak of neutral curve segment $\omega_{c}^{i} \tau=0$ attains higher value. Thus, for this case, the $n_{t h}^{i}$ increases with respect to temperature jump in the low time lag region of the neutral curve. The observations are slightly different for a different flame location, as shown in figure 17 (b). The figure shows that the temperature jump reduces the span of the neutral loop on either side and shifts the peak of the neutral loop down at the same time. This means that for this case the $n_{t h}^{i}$ decreases with respect to temperature jump for all time lags shown in the figure.

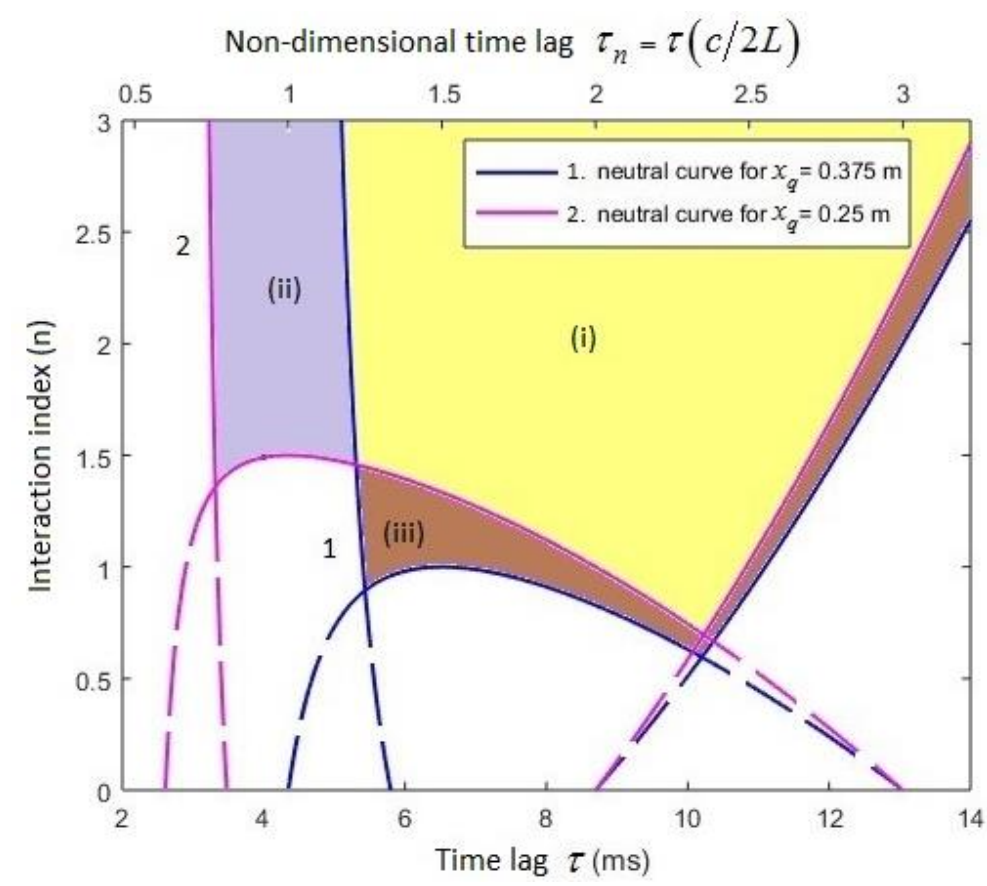

Figure 18: Effect of flame location on stability domain for $m^{i}=1$ and there is no temperature and cross-section jump. The plot is based on (25) and shows two flame locations: $x_{q}=0.375 \mathrm{~m}$ and $0.25 \mathrm{~m}$. The instability domains for the cases $x_{q}=0.375 \mathrm{~m}$ and $x_{q}=0.25 \mathrm{~m}$ are indicated by darker shaded domain (iii) (brown online) and medium density shaded domain (ii) (lavender online), respectively. The common instability domain for these two cases is lightly shaded and 
indicated by (i) (marked in yellow online). Other parameters and notation are the same as in Fig.9.

Figure 18, plotted on the basis of (25), demonstrates the effect of flame location on $n_{t h}^{i}(\tau)$. The figure is drawn for two different flame locations, $x_{q}=0.375 \mathrm{~m}$ and $0.25 \mathrm{~m}$ for uniform temperature and cross-section. Figure 18 shows that as the flame location shifts away from the middle of the resonator (that is $x_{q}=0.375 \mathrm{~m}$ ), the neutral curve stretches to the left and also, the $n_{\text {th }}^{i}$ increases for all time lags shown in the figure. Moreover, it can be shown that in the absence of cross-section and temperature jump, the flame location yielding the minimal $n_{t h}^{i}$ is at the middle of the resonator. The minimal $n_{\text {th }}^{i}$ flame location is independent of cross section jump and depends only on the temperature jump across the flame. In this context, note that the function on the right hand side of equation (25) is a symmetric with respect to $x_{q}=L / 2$. Thus, we can focus only on one half of the resonator for the analysis of the effect of flame location.

A similar analysis can be performed for the growth rates, as well. Figure 19 (a) and (b) shows the effect of cross section jump and temperature jump, respectively, on the growth rate. Growth rates are calculated using the analytical result (39). Figure 19 (a) shows two different cases for time lag, $7 \mathrm{~ms}$ and $9 \mathrm{~ms}$ (the flame is at $x_{q}=0.375 \mathrm{~m}$ ), where, cross-section jumps of $S_{2} / S_{1}=1$ and $S_{2} / S_{1}=2$ are introduced for each case (while the temperature is constant). The growth rate hardly depends on cross section jump for the time lag of $7 \mathrm{~ms}$, but increases significantly with increase of the cross-section for the time lag of $9 \mathrm{~ms}$. Figure 19 (b) shows two different cases for time lag, $6 \mathrm{~ms}$ (the flame is at $x_{q}=0.375 \mathrm{~m}$ ) and $5 \mathrm{~ms}$ (the flame is at $x_{q}=0.25 \mathrm{~m}$ ), where, temperature jumps of $T_{2} / T_{1}=1$ (or, $c_{2} / c_{1}=1$ ) and $T_{2} / T_{1}=4$ (or, $c_{2} / c_{1}=2$ ), are introduced for each case (while the effect of cross-section jump across flame is neglected). The account of temperature jump reduces the growth rate for a time lag of $6 \mathrm{~ms}$ and increases it significantly for a time lag of $5 \mathrm{~ms}$.

Thus, by manipulating the system parameters like cross section jump, temperature jump and flame location, one can indeed increase/lower $n_{\text {th }}^{i}$ and decrease/increase the growth rate, and hence, dampen/instigate the intrinsic mode instability. 


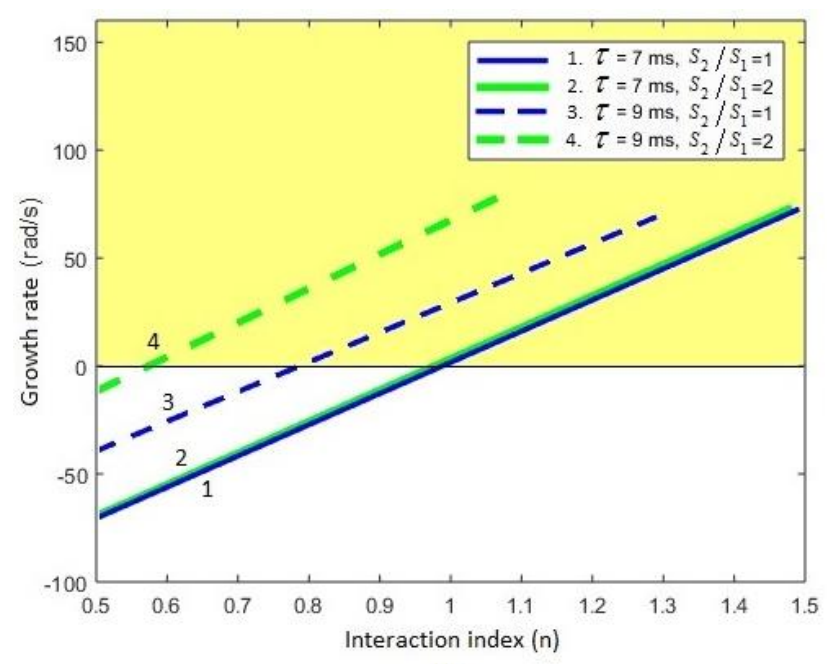

(a)

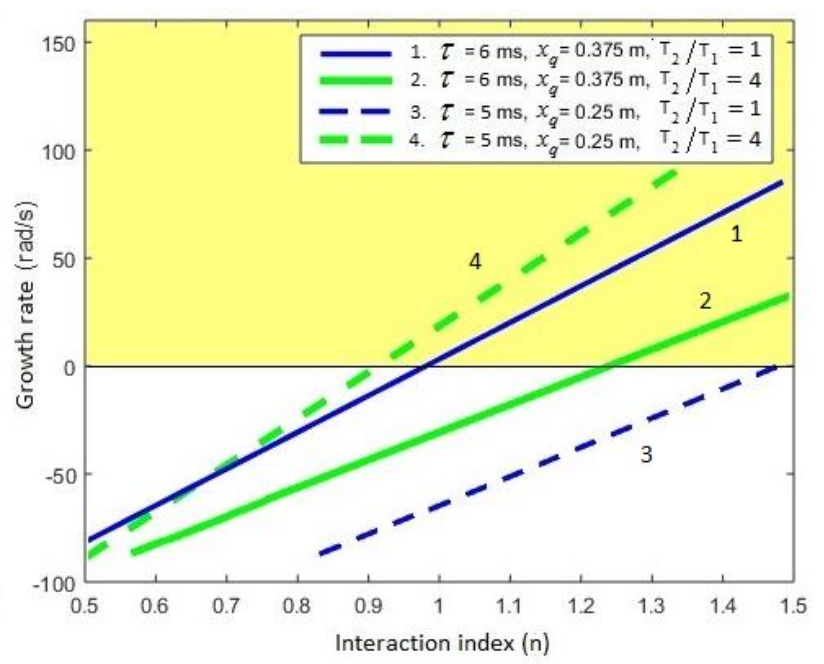

(b)

Figure 19: Effect of cross-section expansion and temperature jump on growth rates for $m^{i}=1$ (based on (39)). (a) Two different time lags (7 and $9 \mathrm{~ms}$ ) are indicated by solid and dashed lines. Two values of the cross section jump ( $S_{2} / S_{1}=1$ and $\left.S_{2} / S_{1}=2\right)$ are marked dark (blue online) and light (green online). The effect of temperature jump across flame is neglected in (a). (b) Two different time lags at different flame locations ( $6 \mathrm{~ms}$ at $x_{q}=0.375 \mathrm{~m}$ and $5 \mathrm{~ms}$ at $x_{q}=0.25 \mathrm{~m}$ ) are

plotted by solid and dashed lines. The growth/decay rate dependence on $n$ for two values of temperature jumps $T_{2} / T_{1}=1$ (or, $c_{2} / c_{1}=1$ ) and $T_{2} / T_{1}=4$ (or, $c_{2} / c_{1}=2$ ) are marked in dark (blue online) and light (green online). The effect of cross-section jump across flame is neglected in (b).

Other parameters and notation are the same as in Fig.9. The domain of instability is lightly shaded (marked in yellow online).

\section{Concluding remarks}

In the present work we have examined analytically flame intrinsic modes within the framework of the simplest 1-D acoustic model of a combustor with $n-\tau$ heat release. For the sake of certainty, we considered a quarter wave resonator, although, our approach is also applicable to other configurations of combustors, as well. The main results could be summarized as follows:

(i) We answered an outstanding question on where unstable intrinsic flame modes are coming from. Our analysis shows that for any nonzero $n$ and $\tau$ there are always an infinite number of intrinsic modes present in any combustor. For small values of $n$ a simple explicit dispersion relation (19a) describes a set of strongly damped modes. To leading order, the real parts of the frequencies $\operatorname{Re}\left(\omega^{i}\right)=\left(2 m^{i}+1\right)(\pi / \tau)$, where $m^{i}$ is the mode number, do not depend on the characteristics of the resonator and the flame position. This is in contrast with the acoustic modes whose frequencies are primarily determined by the resonator length, $L$ ( $\omega_{a}=\left(2 m_{a}+1\right)(\pi c / 2 L)$ (for $\left.m_{a}=0,1,2 \ldots\right)$ for a quarter-wave resonator). For small $n$, the decay rate of the intrinsic modes does not depend on the mode number and is inversely proportional to 
$\tau$. The decay rate logarithmically depends on $n$. For the weak flame regime characterized by small $n$, the heavily damped nature of these modes makes it practically impossible to detect them in combustion systems. The strong decay also makes these modes insensitive to the flame location and the specific properties of the combustor boundaries. Hence, to leading order the modes in any combustor coincide with the intrinsic modes in the infinite tube with anechoic boundary conditions. With increase of $n$, the real part of the frequency shifts only very slightly, unless there is intrinsic-acoustic mode coupling or linear stability of the intrinsic mode for all $n$, and, thus, remains insensitive to all parameters of the system but $\tau$, while, the imaginary part depends on all parameters of the combustor and varies significantly.

(ii) For each intrinsic mode for certain bands of $\tau$, there is a threshold in $n$ above which the mode becomes unstable. The threshold depends on the characteristics of the combustor and flame position, as explicitly given by expression (25). For each intrinsic mode the convoluted neutral curve separating stability and instability domains is made of segments of three types specified by the equations $\omega_{c}^{i} \tau=0, \omega_{c}^{i} \tau=\pi$ and $\omega_{c}^{i} \tau=-\pi$, where $\omega_{c}^{i}$ is the discrepancy between the real part of the frequency on the neutral curve and the real part of the frequency in the limit of small $n$. The intricate shape of the instability domain composed of infinite number of loops of various scales has been revealed. Remarkably, on the long stretches of the neutral curve with the lowest threshold in $n$, the frequency exactly equals the real part of the frequency in the limit of small $n$. On the neutral curve, the instability frequency is independent of the flame location and the combustor parameters (except $\tau$ ) and the mode completely decouples from the environment. This astonishing analytical result has been validated numerically. The fact that the frequencies of marginally unstable intrinsic modes are determined solely by $\tau$ suggests that there are natural oscillations in the subsystem fuel injector-flame; for some combustors determined by the ratio of the distance from fuel injector to flame front and the mean flow velocity.

(iii) Crucially, the threshold value of $n$ might be much smaller for a quarter wave resonator compared to the case with the anechoic boundary condition [9]. Thus, in a closed-open combustor intrinsic modes might attain instability at a much lower value of $n$. The analytical results for the neutral curve are exact and could be used for testing complex software packages.

(iv) The growth/decay rates in the vicinity of the neutral curve have been found analytically and validated numerically. A stability envelope has been introduced to provide a coarse grained stability diagram on the $n-\tau$ plane with all intrinsic modes taken into account. Below this stability envelope, all modes are stable, above this envelope all modes are unstable and within the stability envelope, we have conditional stability domain. A simple technique has been developed to estimate the maximal growth rate at any point on the $n-\tau$ plane.

(v) The dependence of the instabilities on the key parameters of the combustor (the flame location, the cross-section and temperature jumps across the flame) has been described analytically. On this basis by manipulating the system parameters like cross section jump, 
temperature jump and flame location, one can indeed increase/lower the $n_{\text {th }}^{i}$ and increase/decrease the growth rate and hence, prevent/instigate the intrinsic mode instability.

(vi) The question on when acoustic or intrinsic modes are more important in combustion instabilities remains outstanding. Both scenarios are possible. Obviously, the intrinsic modes can be dominant when all acoustic modes are decaying. Such a possibility is illustrated by figure 20 . A sample contour plot of $|f(\omega)|$ is drawn for $\tau=2.2 \mathrm{~ms}, n=1.1$ for the combustor parameters specified in figure 3. The first and second intrinsic mode (indicated by triangles) are unstable in this parameter range, whereas, all the acoustic modes (indicated by diamonds) decay simultaneously, making the intrinsic mode the main factor in combustion instability. To observe intrinsic instabilities in a combustor the following conditions should be satisfied:

(a) $\quad n$ should exceed the instability threshold specified by equation (25);

(b) in general, to avoid being overshadowed by acoustic modes, the acoustic modes should be subdued, which can be ensured, e.g. by the 'out of phase' relationship between the pressure and heat release rate perturbation according to the Rayleigh criterion. However, we can also have scenarios where both intrinsic and acoustic modes are simultaneously unstable [28]. Acoustic modes can be also subdued due to reduced acoustic reflections from the combustor ends. However, since in this work we have not considered non-ideal end conditions, we can mention this scenario only as an educated guess supported by the findings of Hoeijmakers et al. [19].

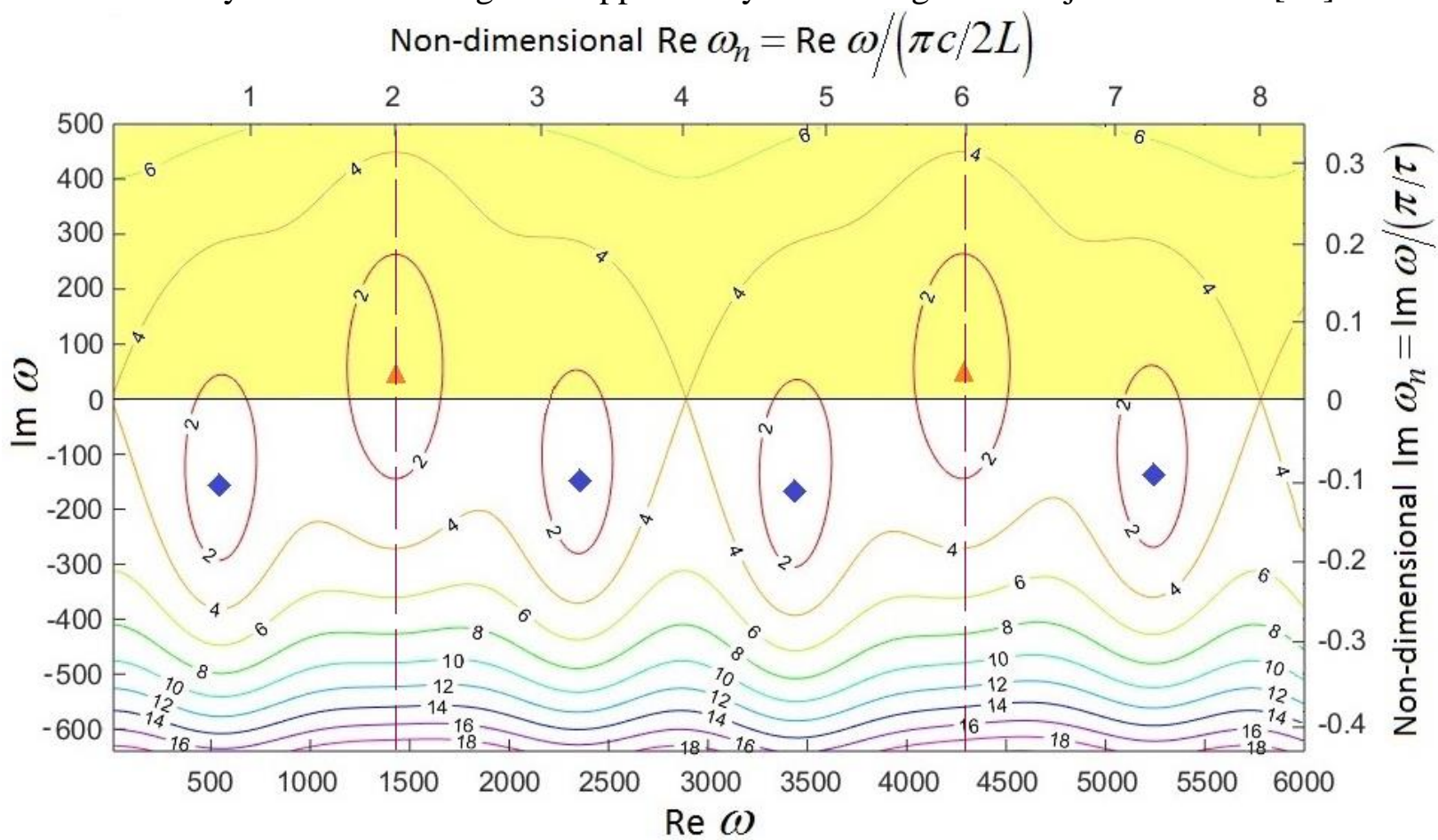

Figure 20: Contour plot of $|f(\omega)|$ for $\tau=2.2 \mathrm{~ms}$ and $n=1.1, x_{q}=L / 2$ and no temperature and cross-section jump. The domain of instability is lightly shaded (marked in yellow online).

Diamonds (blue online) and triangles (orange online) represent the acoustic and intrinsic modes, respectively. Other parameters are the same as in Fig.3. 
The present work was confined to analytic study of linear intrinsic flame modes per se within the framework of the simplest $n-\tau$ model of a closed-open combustor and from our perspective it answered all the questions we aimed at within the framework of this setting. Now we briefly mention a few possible extensions of this work. We mentioned in passing in $\$ 4.2$ that in our numerical analysis of the dispersion relation we encountered situations where acoustic and intrinsic modes are strongly coupled, which suggests a novel alternative route to instability in which intrinsic modes play a key role. We have not explored it in this work. A detailed study of this route requires a dedicated study. A more comprehensive picture of the combustor instabilities which will integrate all types of linear instabilities (the classical acoustic modes, the intrinsic modes studied here and coupled acoustic-intrinsic modes) can be built as a result.

The $n-\tau$ model itself could and should be improved by taking into account the randomly distributed delay, which has been successfully done for acoustic modes (e.g. [29], [30], [31]). Emmert et al. [14] studied intrinsic modes considering a realistic model for the flame dynamics with distributed delays. Mean flow turbulence is a possible source of distributed time delay (another possible source could be advective dispersion), which tends to dampen the acoustic instability. Since most of the industrial combustors work in turbulent regime, we could expect such setups to be less prone to intrinsic instability than laminar setups. How much less and how does it depend on characteristics of turbulence and combustor - remains to be quantified. It seems possible (and desirable) to describe this effect analytically employing our approach.

The present approach can be also directly applied to combustors with open-open end conditions for which the exact decoupling of intrinsic instability frequency from the combustor parameters also occurs. It can be also easily extended to combustor with non-ideal end conditions, as well as to multitude of other systems, which could be considered as perturbed versions of the strongly idealized model of closed-open combustor employed in this work. Once the clarity within the framework of linear models is achieved a study of nonlinear dynamics of intrinsic modes and their nonlinear interactions with acoustic modes will be a natural next step opening a vast new field. The analytical observations made in this paper provided the first overall map of intrinsic instabilities, which opens a possibility for better targeted experimental studies of the nature of intrinsic modes and applicability of specific idealizations adopted in the theoretical model.

\section{Acknowledgements}

The authors are grateful to Prof. Maria Heckl for stimulating discussions. The work is a part of the Marie Curie Initial Training Network Thermo-acoustic and aero-acoustic nonlinearities in green combustors with orifice structures (TANGO). We gratefully acknowledge the financial support from the European Commission under call FP7-PEOPLE-ITN-2012.

\section{REFERENCES}

[1] K. Mcmanus, T. Poinsot, S.M. Candel, A review of active control of combustion instabilities, Prog. Energy Combust. Sci. 19 (1993) 1-29. 
[2] G.B. Warren, Fuel-economy gains from heated lean air-fuel mixtures in motorcar operation, 65-WA/APC-2. Mech. Eng. (1966) 84.

[3] R.C. Lee, D.B. Wimmer, Exhaust emission abatement by fuel variations to produce lean combustion, SAE Technical (1968), 680769.

[4] T. Lieuwen, V. Yang, Combustion instabilities in gas turbine engines: Operational experience, fundamental mechanisms, and modelling, Progress in Astronautics and Aeronautics, AIAA, 2005.

[5] T. Poinsot, D. Veynante, Theoretical and numerical combustion, R. T. Edwards, third edition, 2011.

[6] T. Schuller, D. Durox, P. Palies, S. Candel, Acoustic decoupling of longitudinal modes in generic combustion systems, Combust. Flame 159 (2012) 1921-1931.

[7] A.P. Dowling, The calculation of thermo-acoustic oscillations, J. Sound Vib. 180 (1995) 557581.

[8] A.P. Dowling, S.R. Stow, Acoustic analysis of gas turbine combustors, J. Propul. Power 19 (2003) 751-764.

[9] M. Hoeijmakers, V. Kornilov, I.L. Arteaga, P. Goey, H. Nijmeijer, Intrinsic instability of flame-acoustic coupling, Combust. Flame 161 (2014) 2860-2867.

[10] M. Hoeijmakers, Flame-acoustic coupling in combustion instabilities, $\mathrm{PhD}$ thesis, Technical University of Eindhoven, 2014.

[11] S. Bomberg, T. Emmert, W. Polifke, Thermal vs acoustic response of velocity sensitive premixed flames, Proc. Combust. Inst. 35 (2014) 3185-3192.

[12] A. Gentemann, W. Polifke, Scattering and generation of acoustic energy by a premix swirl burner, ASME Turbo Expo (2007), paper GT2007-27238.

[13] R. Kaess, W. Polifke, T. Poinsot, N. Noiray, D. Durox, T. Schuller, S. Candel, CFD based mapping of the thermo-acoustic stability of a laminar premix burner, Proceedings of the Summer Program (2008) 289-302.

[14] T. Emmert, S. Bomberg, W. Polifke, Intrinsic thermo-acoustic instability of premixed flames, Combust. Flame 162 (2015) 75-85.

[15] E. Courtine, L. Selle, F. Nicoud, W. Polifke, C. Silva, Bauerheim M., Poinsot T., Causality and intrinsic thermo-acoustic instability modes, Proceedings of the Summer Program (2014) 169-178.

[16] E. Courtine, L. Selle, T. Poinsot, DNS of intrinsic thermoacoustic modes in laminar premixed flames, Combust. Flame 162 (2015) 4331-4341.

[17] C.F. Silva, T. Emmert, S. Jaensch, W. Polifke, Numerical study on intrinsic thermo-acoustic instability of a laminar premixed flame, Combust. Flame 162 (2015) 3370-3378.

[18] T. Emmert, S. Bomberg, S. Jaensch, W. Polifke, Acoustic and intrinsic thermo-acoustic modes of a premixed combustor, Proc. Combust. Inst. 36 (2016) 3835-3842. 
[19] M. Hoeijmakers, V. Kornilov, I.L. Arteaga, P. Goey, H. Nijmeijer, Flame dominated thermo-acoustic instabilities in a system with high acoustic losses, Combust. Flame 169 (2016) 209-215.

[20] L. Crocco, S.I. Cheng, Theory of combustion instability in liquid propellant rocket motors, Butterworths Scientific Publications, 1956.

[21] N. K. Mukherjee, M. A. Heckl, V. Shrira, Analysis of flame intrinsic modes in a resonator, International Congress on Sound and Vibration 23 (2016), paper 780.

[22] L.E. Kinsler, A.R. Frey, A.B. Coppens, J.V. Sanders, Fundamentals of Acoustics, John Wiley \& Sons, Fourth edition, 2000.

[23] M. Bauerheim, F. Nicoud, T. Poinsot, Theoretical analysis of the mass balance equation through a flame at zero and non-zero Mach numbers, Combust. Flame 162 (2015) 60-67.

[24] K. Truffin, T. Poinsot, Comparison and extension of methods for acoustic identification of burners, Combust. Flame 142 (2005) 388-400.

[25] R. Vishnu, R. I. Sujith, P. Aghalayam, Role of flame dynamics on the bifurcation characteristics of a ducted V-flame, Combust. Sci. Technol. 187 (2015) 894-905.

[26] N. Mukherjee, M. A. Heckl, A. Bigongiari, R. Vishnu, S. Pawar, R. I. Sujith, Nonlinear dynamics of a laminar V flame in a combustor, International Congress on Sound and Vibration 22 (2015), paper 960.

[27] M.L. Munjal, Acoustics of Ducts and Mufflers, John Wiley, New York, 1987.

[28] C. F. Silva, M. Merk, T. Komarek, W. Polifke, The contribution of intrinsic thermoacoustic feedback to combustion noise and resonances of a confined turbulent premixed flame, Combust. Flame 182 (2017) 269-278.

[29] T. Sattelmayer, Influence of the combustor aerodynamics on combustion instabilities from equivalence ratio fluctuations, International Gas Turbine and Aero Engine Congress \& Exposition ASME (2000), paper 2000-GT-0082

[30] W. Polifke, J. Kopitz, A. Serbanovic, Impact of the fuel time lag distribution in elliptical premix nozzles on combustion stability, American institute of aeronautics and astronautics (2001), paper 2104.

[31] B. Schuermans, V. Bellucci, F. Guethe, F. Meili, P. Flohr, C.O. Paschereit, A detailed analysis of thermoacoustic interaction mechanisms in a turbulent premixed flame, Proceedings of ASME Turbo Expo (2004), paper GT2004-53831.

\section{APPENDIX I}

\section{AI.1. Local maxima of $n_{t h}^{i}$ for neutral curves}

In $\S 4.2$ we have found the value of $n_{t h}^{i}$ at the intersection of two segments of the neutral curve. However, it also might be interesting to have an idea of the maximal/minimal values of $n_{t h}^{i}$ 
corresponding to each segment. We can, at this point, make an attempt to find the values of $\tau$, for which, $n_{\text {th }}^{i}$ attains its local extremum. According to (26), $n_{\text {th }}^{i}$ can be positive only when $\cos \lambda$ is negative, where $\lambda=\left(2 m^{i}+1\right) \pi L /(\tau c)$. Thus, to ensure positivity of $\boldsymbol{n}_{t h}^{i}$ we require,

$$
\cos \left[\left(2 m^{i}+1\right) \pi L /(\tau c)\right]<0 .
$$

This produces an upper and lower bound on the values of $\tau$ for a solution with positive $n_{\text {th }}^{i}$ to exist, where $m$ is a positive integer and can have only odd values to satisfy (AI.1).

$$
(2 L / c)\left(2 m^{i}+1\right) /(2 m+1)<\tau<(2 L / c)\left(2 m^{i}+1\right) /(2 m-1) .
$$

Differentiating both sides of equation (26) for $\omega_{c}^{i} \tau=0$, we get the following equation; where, $\lambda=\zeta / \tau$, and $\zeta$ is a constant, defined as, $\zeta=\left(2 m^{i}+1\right) \pi L / c$,

$$
d n_{\text {th }}^{i} / d \tau=\left\{-2 \sin \lambda /(1-\cos \lambda)^{2}\right\}\left(\zeta / \tau^{2}\right) .
$$

The necessary condition for the extrema of $n_{t h}^{i}, d n_{t h}^{i} / d \tau=0$, requires, $\sin \lambda=0$. This generates an explicit solution for $\lambda, \lambda=m \pi$, where $m$ according to (AI.2) is a positive odd integer. For these values of $m, \cos \lambda=-1$. Thus, the value of $\tau$ corresponding to an extremum is,

$$
\tau=\left(2 m^{i}+1\right)(L / c)(1 / m) .
$$

Differentiating both sides of equation (AI.3) with respect to $\tau$, we get

$$
d^{2} n_{t h}^{i} / d \tau^{2}=2\left(\zeta^{2} / \tau^{4}\right) \cos \lambda /(1-\cos \lambda)^{2} .
$$

Clearly, $d^{2} n_{t h}^{i} / d \tau^{2}<0$ for $\cos \lambda=-1$. Thus, the values of $\tau$ specified by (AI.4) correspond to maxima. According to (26) the maximal value of $n_{t h}^{i}$ is 1 .

Hence, we have derived an analytical criterion for the positivity of $n_{t h}^{i}$ values and explained the specific feature of the neutral curve showing multiple confined loops of instability. We have also derived a maximum value for $n_{t h}^{i}$ and the corresponding value of $\tau$. The maximal value of $n_{t h}^{i}$, for the segment of neutral curve corresponding to $\omega_{c}^{i} \tau=0$ comes out as 1 . Similarly, for the neutral curve segments corresponding to $\omega_{c}^{i} \tau=\pi$ and $\omega_{c}^{i} \tau=-\pi$, it can be shown that the minimal value for this case is -1 and, hence, these segments of neutral curve do not have any maxima. 


\section{APPENDIX II}

\section{AII.1 Asymptotics for large $\tau$}

As figure 11 suggests, the largest loop of the neutral curve shifts towards larger values of $\tau$, with increase of the mode number. In this section we will find asymptotics of $n_{\text {th }}^{i}$ for large time lag $\tau$, that is $\tau \gg 2 L / c$. Large $\tau$ assumption provides a way to find explicitly the intersection points for the neutral curve segments $\omega_{c}^{i} \tau=0$ and $\omega_{c}^{i} \tau= \pm \pi$ belonging to the largest loop, as shown in figure 9. At the intersection $n_{t h}^{i}$ simultaneously satisfies equations for the neutral curve segments $\omega_{c}^{i} \tau=0$ and $\omega_{c}^{i} \tau= \pm \pi$ given by (26). We begin our analysis with the left intersection: $\omega_{c}^{i} \tau=0$ and $\omega_{c}^{i} \tau=\pi$. On equating $n_{t h}^{i}$ for $\omega_{c}^{i} \tau=0$ and $\omega_{c}^{i} \tau=\pi$ from (26) we get

$$
2 \cos (\lambda) /\{\cos (\lambda)-1\}=2 \cos (\lambda+\delta) /\{1-\cos (\lambda+\delta)\}
$$

where, $\lambda, \delta$ and normalized time lag $\tau_{n}$ are:

$$
\lambda=\left(2 m^{i}+1\right) \pi / 2 \tau_{n}, \delta=\pi / 2 \tau_{n} \text { and } \tau_{n}=\tau c / 2 L
$$

We are now focusing on large $\tau$ asymptotics, i.e. $\delta$ in (AII.2) will be treated as a small parameter (i.e. $\delta<<1$, in other words $\tau \gg \pi L / c$. This is the exact range of applicability of large $\tau$ asymptotics.). Using this smallness of $\delta$, equation (AII.1) can be expanded in $\delta$. On retaining just zero and linear terms we get,

$$
2 \cos \lambda-\delta \sin \lambda-2 \cos ^{2} \lambda+2 \delta \sin \lambda \cos \lambda=0,
$$

which immediately yields an explicit expression for $\delta$,

$$
\delta=\left(2 \cos \lambda-2 \cos ^{2} \lambda\right) /\{\sin \lambda(1-2 \cos \lambda)\} .
$$

We can consider $\lambda$, as a sum of a zero order term, $\lambda_{0}$ and a correction $\tilde{\lambda}, \lambda=\lambda_{0}+\tilde{\lambda}$. To leading order in $\delta, \delta=0$ and equation (AII.4) becomes,

$$
2 \cos \lambda_{0}-2 \cos ^{2} \lambda_{0}=0 .
$$

Equation (AII.5) yields two solutions for $\lambda_{0}: \cos \lambda_{0}=0,1$. The $\cos \lambda_{0}=1$ root corresponds to, $\tau_{n} \rightarrow \infty$. Hence, we choose the second solution $\cos \lambda_{0}=0$, which leads to the explicit solution for $\lambda_{0}: \lambda_{0}=\left(2 m_{(+\pi, 0)}+1\right) \pi / 2$, where, $m_{(+\pi, 0)}=0,1,2, \ldots$ The integer $m_{(+\pi, 0)}$ is the intersection point number of the neutral curve segments $\omega_{c}^{i} \tau=0$ and $\omega_{c}^{i} \tau=\pi$, with $m_{(+\pi, 0)}=0$ being the rightmost intersection point, while $m^{i}$ is the mode number. Since not all intersections occur at 
positive $n, m_{(+\pi, 0)}$ cannot take all integer values. This limitation on $m_{(+\pi, 0)}$ will be discussed below. Inserting the two term expansion for $\lambda$ in the form $\lambda=\lambda_{0}+\tilde{\lambda}$ into (AII.4) we get,

$$
\delta=\left\{2 \cos \left(\lambda_{0}+\tilde{\lambda}\right)-2 \cos ^{2}\left(\lambda_{0}+\tilde{\lambda}\right)\right\} /\left\{\sin \left(\lambda_{0}+\tilde{\lambda}\right)\left(1-2 \cos \left(\lambda_{0}+\tilde{\lambda}\right)\right)\right\} .
$$

Due to presumed smallness of $\tilde{\lambda}$ we assume $\cos \tilde{\lambda} \approx 1$ and $\sin \tilde{\lambda} \approx \tilde{\lambda}$. Also, using $\sin \lambda_{0}=(-1)^{m_{(+\pi, 0)}}$, for $\cos \lambda_{0}=0$, we apply the following trigonometric identities in equation (AII.6), $\cos \left(\lambda_{0}+\tilde{\lambda}\right) \approx-(-1)^{m_{(+\pi, 0)}} \tilde{\lambda}, \sin \left(\lambda_{0}+\tilde{\lambda}\right) \approx(-1)^{m_{(+\pi, 0)}}$. On neglecting the higher order terms in $\tilde{\lambda}$, we obtain a simplified expression for $\delta$ from (AII.6), $\delta=-2 \tilde{\lambda}$. Using this form and also, $\lambda_{0}=\left(2 m_{(+\pi, 0)}+1\right) \pi / 2$ and equation (AII.2) we rewrite $\lambda=\lambda_{0}+\tilde{\lambda}$ as follows,

$$
\left(2 m^{i}+1\right) \pi / 2 \tau_{n}=\left(2 m_{(+\pi, 0)}+1\right) \pi / 2-\pi /\left(4 \tau_{n}\right) .
$$

After further simplification, we arrive at an explicit expression for the normalized time lag, $\tau_{n}$, written as $\tau_{n(+\pi, 0)}$, to stress the fact that it corresponds to the intersection point of $\omega_{c}^{i} \tau=0$ and $\omega_{c}^{i} \tau=\pi$, valid for large $\tau$,

$$
\tau_{n(+\pi, 0)}=\left(4 m^{i}+3\right) /\left\{2\left(2 m_{(+\pi, 0)}+1\right)\right\} .
$$

Using (26) $n_{t h}^{i}$ at the intersection can be found as,

$$
n_{t h(+\pi, 0)}^{i}=2 \cos \left\{\left(2 m_{(+\pi, 0)}+1\right)\left(\frac{\pi}{2}\right)\left(1-\frac{1}{4 m^{i}}\right)\right\} /\left[\cos \left\{\left(2 m_{(+\pi, 0)}+1\right)\left(\frac{\pi}{2}\right)\left(1-\frac{1}{4 m^{i}}\right)\right\}-1\right]
$$

To ensure positive $n_{t h(+\pi, 0)}^{i}$, we require $\cos \left\{\left(2 m_{(+\pi, 0)}+1\right)(\pi / 2)\left(1-1 / 4 m^{i}\right)\right\}<0$, thus, $m_{(+\pi, 0)}$ will be restricted to odd integers, i.e. $m_{(+\pi, 0)}=2 j+1$. The even intersection points should be discarded from consideration. On substituting $m_{(+\pi, 0)}=2 j+1$ into (AII.9), we obtain the simplified form,

$$
n_{t h(+\pi, 0)}^{i}=2 \sin \left(3 \pi / 8 m^{i}\right) /\left[\sin \left(3 \pi / 8 m^{i}\right)+1\right] .
$$

This shows how $n_{t h(+\pi, 0)}^{i}$ tends to zero as $m^{i}$ increases. Further, for $m^{i} \gg>1$, $\sin \left(3 \pi / 8 m^{i}\right) \approx 3 \pi / 8 m^{i}$. Thus, for large $m^{i}$ (AII.10) reduces to,

$$
n_{t h(+\pi, 0)}^{i} \approx 6 \pi /\left(3 \pi+8 m^{i}\right)=2 \pi /\left(\pi+8 m^{i} / 3\right) .
$$


Similar consideration of the intersections of the neutral curve segments $\omega_{c}^{i} \tau=0$ and $\omega_{c}^{i} \tau=-\pi$ yields,

$$
\tau_{n(-\pi, 0)}=\left(4 m^{i}+1\right) /\left\{2\left(2 m_{(-\pi, 0)}+1\right)\right\} .
$$

Here $m_{(-\pi, 0)}$ is an integer indicating the intersection point number of the neutral curve segments intersections $\omega_{c}^{i} \tau=0$ and $\omega_{c}^{i} \tau=-\pi$, with $m_{(-\pi, 0)}=0$ being the rightmost intersection point, while $m^{i}$ is the mode number. However, in contrast to the previous case, to ensure positive value of $n_{t h(-\pi, 0)}^{i}$, we require $\cos \left\{\left(2 m_{(-\pi, 0)}+1\right)(\pi / 2)\left(1+1 / 4 m^{i}\right)\right\}<0$. Hence, the value of $m_{(-\pi, 0)}$ will be restricted to even integers, i.e. $m_{(-\pi, 0)}=2 j$, whereas, the odd intersection points should be discarded from consideration for this case. Equivalent to (AII.10), we can arrive at an identical expression for $n_{t h}^{i}$ in this case, as well,

$$
n_{t h(-\pi, 0)}^{i}=2 \sin \left(\pi / 8 m^{i}\right) /\left[\sin \left(\pi / 8 m^{i}\right)+1\right] .
$$

This expression quantifies how $n_{t h(-\pi, 0)}^{i}$ tends to zero as $m^{i}$ increases. A comparison of (AII.10) and (AII.13) shows that $n_{t h(-\pi, 0)}^{i}<n_{t h(+\pi, 0)}^{i}$. Thus the intersection points of neutral curve segments for this case correspond to lower value of $n_{t h}^{i}$ compared to the previous case. For large mode numbers we arrive at an expression for $n_{t h(-\pi, 0)}^{i}$ which is identical to (AII.11),

$$
n_{t h(-\pi, 0)}^{i} \approx 2 \pi /\left(\pi+8 m^{i}\right) .
$$

Figure AII.1 shows how the values of $\tau$ and $n_{t h}^{i}$ at the intersection (given by (AII.8), (AII.10), (AII.12), (AII.13), respectively) depend on the mode number. The plots are for two particular intersection numbers: $m_{(+\pi, 0)}=1$ and $m_{(-\pi, 0)}=0$. As mentioned previously, intersection number $m_{(+\pi, 0)}=0$ correspond to negative $n_{t h}^{i}$. Hence, we made plots for the intersection, $m_{(+\pi, 0)}=1$. In figure AII.1(a) the region above $\tau=\pi L / c$ is marked in light grey to indicate extremely optimistic interpretation of the domain of validity of large $\tau$ assumption (recall, the true condition of validity is $\tau \gg \pi L / c$ ). The values of $\tau$ at the intersection of neutral curve segments go up as the mode number increases, while $n_{t h}^{i}$ at the intersection decreases as $\left(2 \pi /\left(\pi+8 m^{i} / 3\right)\right)$ or $\left(2 \pi /\left(\pi+8 m^{i}\right)\right)$ for high mode numbers.

The figure can be also viewed from a different perspective. For any given $\tau$ a straight horizontal line on figure AII.1(a) would give the numbers of modes whose large loops overlap for such a 
combustor. The intersection of this horizontal line with the solid straight line gives in the generic case a non-integer $m^{i}$. That is, this intersection falls in between the extreme right intersections of neutral curve segments for two subsequent modes. In $\$ 4.4$ it is shown that only these two modes are important from the maximal growth rate prediction perspective. All modes with the numbers below these two modes are stable (for large $n$ ) for this $\tau$, while all modes higher than these two have lesser growth rate. For example, $\tau=25 \mathrm{~ms}$ intersection of $\omega_{c}^{i} \tau=0$ and $\omega_{c}^{i} \tau=-\pi$ falls between the third and fourth intrinsic modes, whereas, the intersection of $\omega_{c}^{i} \tau=0$ and $\omega_{c}^{i} \tau=\pi$ falls between eighth and ninth intrinsic modes, as shows the black dashed line projections on the horizontal axis of figure (AII.1a) and/or relation (AII.8, AII.12). Hence, it is these two modes which are of crucial importance from the growth rate prediction perspective. Figure AII.1(b) also shows that the value of $n_{t h}^{i}$ at $\omega_{c}^{i} \tau=0, \omega_{c}^{i} \tau=-\pi$ intersection is always lower than $n_{t h}^{i}$ at $\omega_{c}^{i} \tau=0, \omega_{c}^{i} \tau=\pi$ intersection, in accordance with (AII.10) and (AII.13). Previously, considering figure 8 we noticed that for the second mode $n_{t h}^{i}$ is minimal at the intersection of the $\omega_{c}^{i} \tau=0$, $\omega_{c}^{i} \tau=-\pi$ segments of the neutral curve for the extreme right loop in the curve. This proved to be the case for all modes. Here in, figure AII.1 we also plot the exact numerical solutions for segments of neutral curve and tracking down manually the points of their intersections. The agreement between the "exact" numerical results shown in figure (AII.1) by circles and the analytical predictions is remarkable, given that the large $\tau$ asymptotics is not formally applicable in the left corner of these figures.

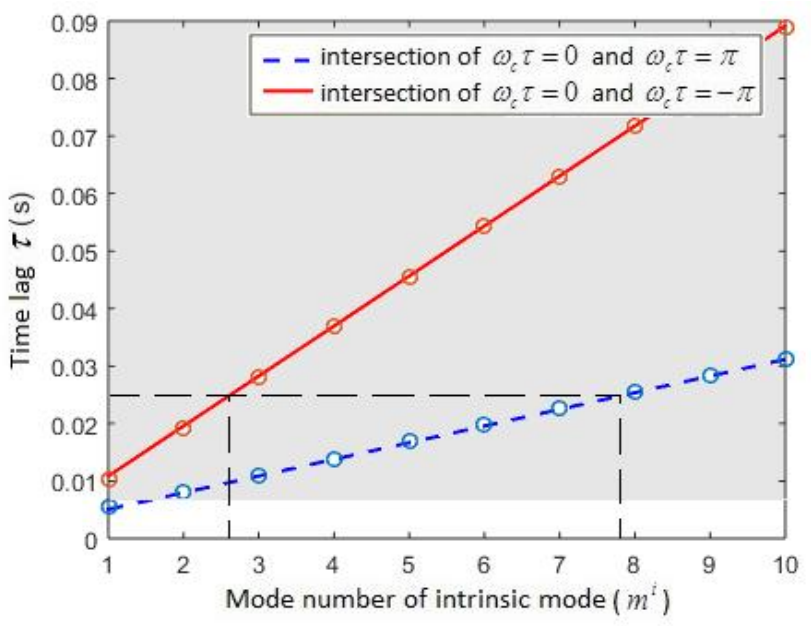

(a)

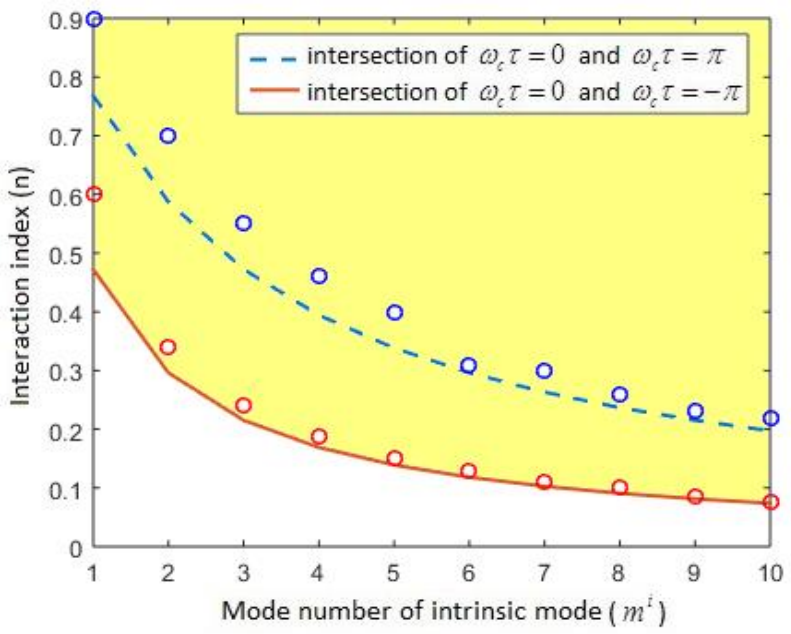

(b)

Figure AII.1: (a) Values of $\tau$ at the intersections of the neutral curve segments $\omega_{c}^{i} \tau=0$ and $\omega_{c}^{i} \tau= \pm \pi$ vs mode number of intrinsic mode. Solid line (red online) and dashed line (blue online) show the large $\tau$ asymptotic solution (AII.12, AII.8) for the intersections $\omega_{c}^{i} \tau=0$, $\omega_{c}^{i} \tau=-\pi$ (when $m_{(-\pi, 0)}=0$ ) and $\omega_{c}^{i} \tau=0, \omega_{c}^{i} \tau=\pi$ (when $m_{(+\pi, 0)}=1$ ) segments, respectively.

Circles (red and blue in online) show their numerical counterparts found by plotting (26) and tracking down manually the points of intersection of the corresponding neutral curve segments. 
The light grey color represents the region where the large $\tau$ assumption might be applicable. (b) $n_{\text {th }}^{i}$ at the intersections of neutral curve segments $\omega_{c}^{i} \tau=0$ and $\omega_{c}^{i} \tau= \pm \pi$ vs mode number of intrinsic mode. Solid line (red online) and dashed line (blue online) show analytical solution (AII.13, AII.10) for $\omega_{c}^{i} \tau=0, \omega_{c}^{i} \tau=-\pi$ (for $m_{(-\pi, 0)}=0$ ) and $\omega_{c}^{i} \tau=0, \omega_{c}^{i} \tau=\pi$ (for $m_{(+\pi, 0)}=1$ ) intersections, respectively. Circles (red and blue in online) show their numerical counterparts as in panel (a). The instability domain is lightly shaded (marked in yellow online). The first intrinsic mode is not shown.

Thus, to avoid dealing with large $m_{(+\pi, 0)}, m_{(-\pi, 0)}$ and large $\tau$ regimes head on, we found simple analytical description of the points of intersection of the segments of neutral curves $\omega_{c}^{i} \tau=0$ and $\omega_{c}^{i} \tau=\pi$, as well as, $\omega_{c}^{i} \tau=0$ and $\omega_{c}^{i} \tau=-\pi$, assuming large $\tau$. At the intersection of neutral curve segments, $n_{t h}^{i}$ decreases with increase of mode number. $n_{t h}^{i}$ at the intersection of $\omega_{c}^{i} \tau=0$ and $\omega_{c}^{i} \tau=\pi$ is always larger than $n_{t h}^{i}$ at the intersection of $\omega_{c}^{i} \tau=0$ and $\omega_{c}^{i} \tau=-\pi$. The extreme right intersection point of $\omega_{c}^{i} \tau=0$ and $\omega_{c}^{i} \tau=-\pi$ (that is, the intersection of the large loop with $m_{(-\pi, 0)}=0$ as given by (AII.12)) corresponds to the lowest value of $n_{t h}^{i}$ for any fixed mode number. 